\title{
EQUIVALENCE OF NEIGHBORHOODS OF EMBEDDED COMPACT COMPLEX MANIFOLDS AND HIGHER CODIMENSION FOLIATIONS
}

\author{
XIANGHONG GONG ${ }^{\dagger}$ AND LAURENT STOLOVITCH ${ }^{\dagger \dagger}$
}

\begin{abstract}
We consider an embedded $n$-dimensional compact complex manifold in $n+d$ dimensional complex manifolds. We are interested in the holomorphic classification of neighborhoods as part of Grauert's formal principle program. We will give conditions ensuring that a neighborhood of $C_{n}$ in $M_{n+d}$ is biholomorphic to a neighborhood of the zero section of its normal bundle. This extends Arnold's result about neighborhoods of a complex torus in a surface. We also prove the existence of a holomorphic foliation in $M_{n+d}$ having $C_{n}$ as a compact leaf, extending Ueda's theory to the high codimension case. Both problems appear as a kind linearization problem involving small divisors condition arising from solutions to their cohomological equations.
\end{abstract}

\section{Contents}

1. Introduction 2

2. Full linearizations, horizontal foliations, and vertical linearizations 7

2.1. Transition functions $\quad 7$

2.2. The equivalence of transition functions 9

2.3. The full Linearization of a neighborhood 11

2.4. Horizontal foliations and vertical trivializations 12

2.5. The vertical linearization 13

2.6. Coboundary operators in symmetric powers and coordinates 14

2.7. Formal obstructions in cohomology groups 16

2.8. Automorphisms of neighborhood of the zero section of flat vector bundles 17

2.9. Formal coordinates in the absence of formal obstructions 20

3. A majorant method for the vertical linearization 23

3.1. Conjugacy equations and cohomological equations 24

3.2. A modified Fischer norm for symmetric powers 25

3.3. A majorization in the modified Fischer norm for the vertical linearization 27

4. A majorant method for the full linearization with a unitary normal bundle 33

5. The full linearization 38

5.1. Domains for iteration and the Donin condition 39

Date: July 13, 2020.

2010 Mathematics Subject Classification. 32Q57, 32L30, 32L10, 37F50, 58F36.

Key words and phrases. Neighborhood of a complex manifold, normal bundle, solution of cohomological equations with bounds, holomorphic extension, holomorphic linearization, resonances, small divisors condition, holomorphic foliations.

${ }^{\dagger}$ Partially supported by a grant from the Simons Foundation (award number: 505027). ${ }^{\dagger \dagger}$ Research of L. Stolovitch was supported by ANR grant "ANR-14-CE34-0002-01" for the project "Dynamics and CR geometry". 
5.2. A Newton method for the full linearization 43

5.3. An example of Arnol'd 47

5.4. Counter-examples 49

5.5. A foliation example $\quad 50$

Appendix A. $\quad L^{2}$ bounds of cohomology solutions and small divisors $\quad 51$

A.1. A question of Donin $\quad 51$

A.2. Bounds of solutions of cohomology equations $\quad 52$

A.3. Donin's smoothing decomposition $\quad 55$

$\begin{array}{lll}\text { A.4. Finiteness theorem with bounds } & 58\end{array}$

A.5. Existence of nested coverings 64

$\begin{array}{ll}\text { References } & 69\end{array}$

\section{INTRODUCTION}

We are interested in the classification of the germs of neighborhood of an embedded compact complex manifold $C$ in a complex manifold $M$. Here two germs $(M, C)$ and $(\tilde{M}, C)$ are holomorphically equivalent if there is a biholomorphic mapping $F$ fixing $C$ pointwise and sending a neighborhood $V$ of $C$ in $M$ into a neighborhood $\tilde{V}$ of $C$ in $\tilde{M}$. These considerations can be useful to extend holomorphic objects such as cohomology classes of holomorphic sections of bundles over $C$ or functions on $C$ to a neighborhood of $C$ in $M$. Indeed, it might be that such an extension problem is much easy to solve on an equivalent neighborhood. We are also interested in the existence of a non-singular holomorphic foliation of the germ of neighborhood of $C$ in a complex manifold having $C$ as a compact leaf. We refer to it as a "horizontal foliation".

A neighborhood $V$ of an embedded complex manifold $C_{n}$ in $M_{n+d}$ has local holomorphic charts $\left(h_{j}, v_{j}\right)=\Phi_{j}$ mapping $V_{j}$ onto $\hat{V}_{j}$ in $\mathbf{C}^{n+d}$ with $n=\operatorname{dim} C$. Here $\cup V_{j}$ is a neighborhood of $C$ and $U_{j}:=V_{j} \cap C$ is defined by $v_{j}=0$. The above-mentioned classification of the germs of neighborhoods of $C$ is then the classification of transition functions $\Phi_{k j}:=\Phi_{k} \Phi_{j}^{-1}$ under holomorphic conjugacy $F_{k}^{-1} \Phi_{k j} F_{j}$. To such an embedding, one can associate the normal bundle $N_{C}(M)$ of $C$ in $M$, which has the transition matrices $g_{k j}(p)$, $p \in U_{k} \cap U_{j}$. To this embedding one can associate another natural embedding, namely the embedding of $C$ as the zero section of $N_{C}(M)$. Under a mild assumption, this last embedding $\left(N_{C}(M), C\right)$ naturally serves as a first order approximation of $(M, C)$. Let $\varphi_{j}=\left.\Phi_{j}\right|_{U_{j}}$ and let $\varphi_{k j}=\varphi_{k} \varphi_{j}^{-1}$ be the transition functions of $C$. To have a neighborhood of $C$ in $M$ equivalent to a neighborhood of the zero section in $N_{C}(M)$ is equivalent to seeking $F_{j}$ such that $\hat{\Phi}_{k j}=F_{k}^{-1} \Phi_{k j} F_{j}$ are of the form $N_{k j}\left(h_{j}, v_{j}\right)=\left(\varphi_{k j}\left(h_{j}\right), t_{k j}\left(h_{j}\right) v_{j}\right)$ with $t_{k j}\left(h_{j}\right)=g_{k j}$, the latter being regarded as the transition functions of a neighborhood of the zero section of $N_{C}(M)$. We call this process a "full linearization" of the neighborhood. The above-mentioned "horizontal foliation" will be obtained as a consequence of a "vertical linearization" of the neighborhood which amounts to seeking $F_{j}$ such that $\hat{\Phi}_{k j}=\left(\varphi_{k j}\left(h_{j}\right)+\hat{\phi}_{k j}^{h}\left(h_{j}, v_{j}\right), t_{k j}\left(h_{j}\right) v_{j}\right)$.

Without even considering holomorphic equivalence problem, it is known that there are formal obstructions to linearizing $[15,32]$ or to linearizing vertically [40] a neighborhood; see 
section 2. Part of the Grauert formal principle $[5,12,17,27]$ is to seek geometry conditions that ensure a holomorphic linearization when the formal obstructions are absent. In this paper, we will obtain linearizations of a neighborhood of an embedded compact complex manifold $C_{n}$ at the absence of formal obstructions under small divisor conditions in the form of bounds of solutions of cohomology equations involving all symmetric powers of $N_{C}^{*}$, the dual of the normal bundle $N_{C}$ of $C_{n}$ in $M_{n+d}$. Because of the very nonlinear nature of the problem, we need to work with a family of nested domains on which we solve and eventually bound the solutions of 1-cohomological equations. Indeed, we are naturally led to consider shrinking of the domains as we need to get estimates of derivatives of sections (by Cauchy estimates for instance). To be more precise, assume that a 1-cocycle $f$ with value in the sheaf of sections of holomorphic bundle (involving symmetric power $S^{m} N_{C}^{*}$ for some $m \geq 2$ ) on $C$ vanishes in the 1 st cohomology group over a covering $\mathcal{W}$. Then there is a 0 -cochain $w$ over $\mathcal{W}$ such that $\delta w=f$. Nevertheless, we need to prove the existence of a (possibly different) solution $u$ satisfying the linear equation $\delta u=f$ and a "linear" estimate of the form $\|u\|_{\mathcal{W}} \leq K\|f\|_{\mathcal{W}}$ (the norm is either $L^{2}$ or the sup-norm). Because of the nonlinear nature of our problem, we need to solve the linear equation iteratively and estimate solutions of the form $\delta u_{m}=F_{m}\left(f_{2}, \ldots, f_{m}, u_{2}, \ldots, u_{m-1}\right), m \geq 2$. Here $F_{m}\left(f_{2}, \ldots, f_{m}, u_{2}, \ldots, u_{m-1}\right)$ is a nonlinear function and vanishes in a first cohomology group. Therefore the bound $K$, depending on $m$, will compound, which leads to a problem on non-linear estimates. Here come some of the main issues : we need that, at the limit, the sequence of nested domains, over which the solutions are estimated iteratively, remains to cover the manifold. And we need to control the growth of the bound $K$ with respect to $m$, that gives rise to the so-called small divisors condition. Therefore, the existence of any bound $K$ for linear solutions $u$ without shrinking the covering $\mathcal{W}$ is a basic question. The latter was solved affirmatively by Kodaira-Spencer [25, eq. (9), p. 499] for the case of line bundles for a general covering. For higher rank vector bundles, we provide a positive solution in the following result :

Proposition 1.1. Let $C$ be a compact complex manifold. There exists a family of coverings $\mathcal{U}^{r}=\left\{U_{j}^{r}\right\}, r_{*} \leq r<r^{*}$, of $C$ such that for any holomorphic vector bundle $E$ over $C$, and each $f \in C^{1}\left(\mathcal{U}^{r^{\prime}}, E\right)$, the space of 1-cochains on $\mathcal{U}^{r^{\prime}}$ of holomorphic sections with values in $E$, satisfying $f=\delta u_{0}$ for some $u_{0} \in C^{0}\left(\mathcal{U}^{r^{\prime}}, E\right)$, there exist $u \in C^{0}\left(\mathcal{U}^{r^{\prime}}, E\right)$ and $v \in C^{0}\left(\mathcal{U}^{r^{\prime \prime}}, E\right)$ such that $\delta u=f$ and $\delta v=f$, and

$$
\begin{gathered}
|u|_{r^{\prime}} \leq K(E)|f|_{r^{\prime}}, \\
|v|_{r^{\prime \prime}} \leq \frac{D(E)}{\left(r^{\prime}-r^{\prime \prime}\right)^{\tau}}|f|_{r^{\prime}} .
\end{gathered}
$$

Here $r^{\prime}, r^{\prime \prime}$ are any numbers satisfying $r_{*}<r^{\prime \prime}<r^{\prime} \leq \tilde{r}<r^{*}$ and $r^{\prime}-r^{\prime \prime} \leq r^{*}-\tilde{r}$, and $\tau, K(E), D(E)$ are independent of $r^{\prime}, r^{\prime \prime}$.

Here, we have used the sup-norm (or $L^{2}$-norm) of cochains of holomorphic sections of bundles (see section A.2 for specific notations). We do not know if $K(E)$ and $D(E)$ are comparable when they are applied to the symmetric powers of $N_{C}^{*}$ except when $N_{C}$ is unitary. Hörmander $[19,33]$ obtained solutions with bounds for cohomology groups with respect to the $\bar{\partial}$ operator acting on the sheaf of $(p, q)$-forms with $L^{2}$ coefficients on $\mathbf{C}^{n}$. 
The estimate (1.2) was proved by Donin [8] for a special family of coverings by the $L^{2}$ theory. He also raised the question if estimate (1.1) exists, i.e. the basic question mentioned above. Proposition 1.1 gives us a more flexible kind of results and ultimately an estimate that holds without any shrinking for higher rank vector bundles via the above mentioned nested coverings. We also use the $L^{2}$-theory. We first obtain (1.2) by Theorem A.9. Then (1.1) is obtained by Lemma A.2. The constant $K(E)$ is defined for the kind of bundles we need in Definition A.5. This is summarized in Theorem A.12. The main results of this paper are based on the existence of nested finite coverings proved in subsection A.5.

Proposition 1.1 will be a useful tool in this paper. We now formulate our main results. We say that $T_{C} M=\left.T M\right|_{C}$ splits if $T_{C} M=T C \oplus N_{C}$ holomorphically. For instance, $T_{C} E$ splits for any holomorphic vector bundle $E$ over $C$. Here and in the sequel, we identify $C$ with the zero section of $E$. We say that $N_{C}$ is flat if the transition matrices of $N_{C}$ are locally constant. We say that $N_{C}$ is unitary if its transition matrices are unitary. Note that the maximum principle implies that a unitary $N_{C}$ is flat. We have the following "vertical linearization" result:

Theorem 1.2. Let $C_{n}$ be a compact submanifold of $M_{n+d}$ with splitting $T_{C} M$ and unitary $N_{C}$. Let $\eta_{0}=1$ and

$$
\eta_{m}:=K\left(N_{C} \otimes S^{m}\left(N_{C}^{*}\right)\right) \max _{m_{1}+\cdots+m_{p}+s=m} \eta_{m_{1}} \cdots \eta_{m_{p}}
$$

where the maximum is taken in $1 \leq m_{i}<m$ for all $i$ and $s \in \mathbb{N}$. Assume that there are positive constants $L, L_{0}$ such that

$$
\eta_{m} \leq L_{0} L^{m}, \quad m=1,2 \ldots
$$

Assume that $H^{0}\left(C, N_{C} \otimes S^{\ell}\left(N_{C}^{*}\right)\right)=0$ for all $\ell>1$. Assume that either $H^{1}\left(\mathcal{U}, N_{C} \otimes\right.$ $\left.S^{\ell}\left(N_{C}^{*}\right)\right)=0$ for all $\ell>1$ or a neighborhood of $C$ is formally vertically linearizable by a formal holomorphic mapping that is tangent to the identity and preserves the splitting of $T_{C} M$. Then the embedding is actually holomorphically vertically linearizable.

When $C$ is a compact holomorphic curve embedded in a complex surface $M$ with a unitary normal bundle $N_{C}$, the above vertical linearization is one of main results in Ueda [40] where $H^{0}\left(C, N_{C} \otimes S^{\ell}\left(N_{C}^{*}\right)\right)=0$ for all $\ell>1$ follows from his small-divisor condition. This has been generalized by $\mathrm{T}$. Koike in higher codimension case under a strong assumption that $N_{C}$ is a direct sum of unitary line bundles [26]. The Ueda theory for codimension-one foliations has also been extended by Claudon-Loray-Pereira-Touzet [6] and Loray-ThomTouzet [29]. We remark that Theorem 1.2 via the flatness of $N_{C}$ ensures the existence of a "horizontal" foliation :

Corollary 1.3. Under assumptions of Theorem 1.2, there exists a neighborhood of $C_{n}$ in $M_{n+d}$ that admits an $n$-dimensional smooth holomorphic foliation having $C_{n}$ as a leaf.

The following results can been understood in the context of the Grauert formal principle for rigidity: If $(M, C)$ is formal equivalent to $\left(N_{C}, C\right)$, then they are holomorphically equivalent under suitable assumptions. We first consider the unitary case.

Theorem 1.4. Let $C_{n}$ be a compact submanifold of $M_{n+d} N_{C}$ is unitary. Let $\eta_{0}=1$ and

$$
\eta_{m}:=\max \left(K\left(N_{C} \otimes S^{m}\left(N_{C}^{*}\right)\right), K\left(T_{C} \otimes S^{m}\left(N_{C}^{*}\right)\right)\right) \max _{m_{1}+\cdots+m_{p}+s=m} \eta_{m_{1}} \cdots \eta_{m_{p}}
$$


where the maximum is taken in $1 \leq m_{i}<m$ for all $i$ and $s \in \mathbb{N}$. Assume that there are positive constants $L, L_{0}$ such that

$$
\eta_{m} \leq L_{0} L^{m}, \quad m=1,2 \ldots
$$

If $T_{C} M$ splits and $H^{1}\left(\mathcal{U}, T_{C} M \otimes S^{\ell}\left(N_{C}^{*}\right)\right)=0$ for all $\ell>1$ or more generally if a neighborhood of $C$ in $M$ is linearizable by a formal holomorphic mapping which is tangent to the identity and preserves the splitting of $T_{C} M$, then there exists a neighborhood of $C$ in $M$ which is holomorphically equivalent to a neighborhood of $C$ (i.e the 0 th section) in $N_{C}$ In that case, we say that the embedding $C \hookrightarrow M$ is holomorphically linearizable.

More generally, the following result treats two more general cases.

Theorem 1.5. Let $C_{n}$ be a compact submanifold of $M_{n+d}$. Suppose that

$$
\sum_{k \geq 1} \frac{\log D_{*}\left(2^{k+1}\right)}{2^{k}}<+\infty,
$$

where $D_{*}\left(2^{k+1}\right)$ is defined by (5.27). Suppose that either $H^{0}\left(C, T C \otimes S^{\ell}\left(N_{C}^{*}\right)\right)=0$ for all $\ell>1$, or $N_{C}$ is flat. Assume further that either $T_{C} M$ splits and $H^{1}\left(\mathcal{U}, T_{C} M \otimes S^{\ell}\left(N_{C}^{*}\right)\right)=0$ for all $\ell>1$ or $(M, C)$ and $\left(N_{C}, C\right)$ are equivalent by a formal holomorphic mapping which is tangent to the identity and preserves the splitting of $T_{C} M$. Then $(M, C)$ and $\left(N_{C}, C\right)$ are actually holomorphically equivalent.

The previous results can be seen as a "full linearization" results. Theorem 1.4 is proved by using a majorant method while Theorem 1.5 is based on a Newton scheme. It is not clear how to compare the two "small divisors conditions" (1.3) and (1.4) althought the counterparts in theory of dynamical systems are equivalent [4]. The formal principle holds in the following cases: $(a)$ negative $N_{C}$ in the sense of Grauert, by results of Grauert [12] and Hironaka-Rossi [17]. In Grauert's case, $C_{n}$ has a system of strictly pseudoconvex neighborhoods and consequently $C_{n}$ is the only compact $n$-submanifold near $C_{n}$. In the same spirit, Savelev proved that all neighborhoods of embeddings of $\mathbb{P}^{1}$ in complex surfaces with a unitary flat normal bundle are holomorphically equivalent [36]. (b) sufficiently positive $N_{C}$ and $\operatorname{dim} C>2$, by a result of Griffiths [15, Thm II (i)] showing that a neighborhood is determined by a finite-order neighborhood. In other words, under this condition the holomorphic classification of neighborhoods is "finitely determined". (c) $H^{1}\left(C, N_{C}\right)=0$ and the case that for each $x \in C$ there is $x^{\prime} \in C$ such that the fiber of $N_{C}$ at $x$ is generated by global sections of $N_{C}$ vanishing at $x^{\prime}$, by a result of Hirschowitz (see [18] for more general results $)^{1}$. (d) 1-positive $N_{C}$, by a result of Commichau-Grauert [7].

We should remark that the above "full linearization" result was obtained by Arnol'd when $C$ is an elliptic curve and $M$ is a surface, where the vanishing of $H^{0}\left(X, T_{C} M \otimes S^{\ell} M\right)$ follows from the non vanishing of "small divisors" [2,3]. Ilyashenko and Pyartli [22] proved an analogous result for special embeddings of the product flat tori under a strong assumption that $N_{C}$ is a direct sum of flat line bundles. We emphasize that in our linearization Theorem 1.5, for general compact manifolds $C_{n}$, we impose the vanishing of $H^{0}\left(X, T_{C} M \otimes\right.$

\footnotetext{
${ }^{1}$ Recently, Jun-Muk Hwang proved instances of Hirschowitz's conjecture on the Formal Principle [21]. The authors thank Takeo Ohsawa for acknowledging this work.
} 
$\left.S^{\ell} M\right)$ for all integers $\ell \geq 2$ whereas there is no restriction on $H^{0}$ when $C$ is affine and $N_{C}$ is flat.

As a simple consequence, we have the following

Corollary 1.6. Under assumptions of Theorem 1.5 on $C$ and $M$, any holomorphic section of a holomorphic vector bundle $E$ over $C$ extends to a holomorphic section of a holomorphicvector-bundle extension of $E$ over a neighborhood of $C$ in $M$.

Corollary 1.7. Let $C$ be a compact complex manifold. Let $(M, C)$ be equivalent to $(C \times$ $\left.\mathrm{C}^{d}, C\right)$ by a formal holomorphic mapping which is tangent to the identity and preserves the splitting of $T_{C} M$. Suppose that the small-divisor condition in Theorem 1.5 is satisfied. Then $(M, C)$ is holomorphically equivalent to $\left(C \times \mathbf{C}^{d}, C\right)$.

We now give an outline of the paper.

In section 2 we study the formal obstructions to the full linearization and vertical linearization problems. The formal obstructions are known from work of Nirenberg-Spencer [32], Griffiths [15], Morrow-Rossi [30], for the the full linearization problem and by Ueda [40] (see also Neeman [31] and among others) for the vertical linearization problem. The obstructions are described in $H^{1}\left(C, E \otimes S^{\ell} N_{C}^{*}\right)$ for a natural vector bundle $E$ that is either $T_{C} M$ or $N_{C}$. In this paper we emphasize the role of $H^{0}\left(C, T_{C} M \otimes S^{\ell} N_{C}^{*}\right)$. In local dynamical systems, the elements in the analogous group appear as finite symmetries in the Ecalle-Voronin theory [1] and centralizers for the linearizations [11]. The small divisors in local dynamics emerge in the form of the bounds $K\left(N_{C} \otimes S^{\ell} N_{C}^{*}\right)$ and $D\left(T_{C} M \otimes S^{\ell} N_{C}^{*}\right)$ in Proposition 1.1. In work of Arnol'd [2] and Ueda [40], the vanishing condition of the corresponding zero-th cohomology groups is not explicit; however it follows from their small-divisor conditions.

In section 3, we prove Theorem 1.2 by using Ueda's majorization method [40]. In our case the majorization relies on an important tool of the (modified) Fischer norm which is invariant under a unitary change of coordinates. The invariance allows us to overcome the main difficulty in our majorization proof to deal with the transition functions of $N_{C}^{*}$ when they are unitary, but not necessarily diagonal. The (modified) Fischer norms have also been useful in other convergence proofs $[23,28,38]$. In section 4 , we also extend the majorant method to the full linearization problem for the special case where $N_{C}$ is unitary. In section 5, we obtain the full linearization in the general case by introducing a Newton scheme, i.e. a rapid convergence scheme as in Brjuno's work [4]; see also [35,39]. However, we must cope with the domains of transition functions which are not so regular. These domains, when carefully chosen, have nevertheless a disc structure. This allows us to obtain a proof by using sup-norm estimates.

Finally, the paper contains an appendix which has interests in its own right. It has two results, namely the existence of the two bounds stated in Proposition 1.1 and the existence of nested coverings (see Definition A.1). The existence of bound $K(E)$ was employed by Ueda [40] through the complete system of Kodaira-Spencer [25] when $\operatorname{dim} C=1$ and $\operatorname{codim}_{M} C=1$. We will prove Proposition 1.1 by using some techniques developed by Donin [8]. Our proof also relies on a "quantified" version of Grauert-Remmert finiteness theorem [14]. The existence of bound $D\left(E^{\prime} \otimes S^{\ell} E^{\prime \prime}\right)$ was proved by Donin [8] for the socalled "normal" coverings. We have used nested coverings in the proof of Proposition 1.1 
as well as the convergence proof in Theorem 1.5. We believe that the methods and tools developed in this article will be useful for other kinds of problems.

\section{Full linearizations, Horizontal Foliations, AND VERTiCAl LiNEARizATiOns}

In this section, we describe the problem of equivalence of a neighborhood of a complex compact submanifold $C$ of $M$ with a neighborhood of the zero section in the normal bundle of $C$ in $M$ as a "full" linearization problem of the transition functions of this neighborhood. We also describe the existence of a holomorphic foliation of a neighborhood of $C$ having $C$ as a leave as a consequence of a vertical linearization problem of the transition functions of this neighborhood.

We will first describe the formal coordinate changes in terms of cohomological groups of holomorphic sections of a suitable sequence of holomorphic vector bundles.

2.1. Transition functions. We recall basic facts on vector bundles, which we refer to [16, Chap. 0, Sect. 5].

We first set up notation. If a vector space $E$ has a basis $e=\left\{e_{1}, \ldots, e_{d}\right\}$, then a vector $v$ in $E$ can be expressed as

$$
v=\xi^{\mu} e_{\mu}, \quad \xi=\left(\xi^{1}, \ldots, \xi^{d}\right)^{t} .
$$

Here, we use the summation notation: $\xi^{\mu} e_{\mu}$ stands for $\sum_{\mu=1}^{d} \xi^{\mu} e_{\mu}$. The $\xi^{\mu}$ 's are the coordinates or components of $v$ in the basis $e$.

We recall that a holomorphic vector bundle $\mathbf{E}$ over a complex manifold $X$ is defined by a projection $\pi: \mathbf{E} \rightarrow X$ and holomorphic trivializations $\Psi_{j}: \pi^{-1}\left(D_{j}\right) \rightarrow D_{j} \times \mathbf{C}^{r}$ such that each $\Psi_{j}: \pi^{-1}\left(D_{j}\right) \rightarrow D_{j} \times \mathbf{C}^{r}$ is a biholomorphism, and $\Psi_{j}\left(\mathbf{E}_{p}\right)=\{p\} \times \mathbf{C}^{r}$ for $\mathbf{E}_{p}:=\pi^{-1}(p)$. Furthermore $\left\{D_{j}\right\}$ is an open covering of $X$ and the maps $\Psi_{k j}=\Psi_{k} \Psi_{j}^{-1}: D_{k} \cap D_{j} \times \mathbf{C}^{r} \rightarrow$ $D_{k} \cap D_{j} \times \mathbf{C}^{r}$ satisfy

$$
\Psi_{k j}\left(p, \xi_{j}\right)=\left(p, g_{k j}(p) \xi_{j}\right)
$$

where $g_{k j}$ are transition matrices which are holomorphic and invertible. Thus for $\xi_{k}^{\mu} e_{k, \mu}=$ $\xi_{j}^{\mu} e_{j, \mu}$, we have

$$
\begin{array}{cc}
\xi_{k}^{\mu}=g_{k j, \nu}^{\mu} \xi_{j}^{\nu}, & e_{j, \mu}=g_{k j, \mu}^{\nu} e_{k, \nu}, \\
\xi_{k}=g_{k j} \xi_{j}, & e_{k}=\left(g_{k j}^{-1}\right)^{t} e_{j} .
\end{array}
$$

They satisfy the cocycle conditions,

$$
g_{k j} g_{j k}=I, \quad \text { on } D_{k} \cap D_{j} ; \quad g_{k i} g_{i j}=g_{k j}, \quad \text { on } D_{k} \cap D_{j} \cap D_{i} .
$$

We also need to consider the dual bundle $E^{*}$. Let $e_{j}^{*}$ be the basis dual to $e_{j}$ so that $\left(e_{j, \mu}^{*}\left(e_{j, \nu}\right)\right)$ is the identity matrix. Suppose $\zeta_{j}^{\mu} e_{j, \mu}^{*}=\zeta_{k}^{\mu} e_{k, \mu}^{*} \in E^{*}$. Corresponding to (2.3), we have

$$
e_{k}^{*}=g_{k j} e_{j}^{*}, \quad \zeta_{k}=\left(g_{k j}^{-1}\right)^{t} \zeta_{j} .
$$

Let us also express transition functions for various vector bundles in coordinate charts as above. Let $C_{n}$ be a compact complex manifold embedded in complex manifold $M_{n+d}$. We 
cover a neighborhood of $C$ in $M$ by open sets $V_{j}$ so that we can choose coordinate charts $\left(z_{j}, w_{j}\right)$ on $V_{j}$ for $M$ such that

$$
U_{j}:=C \cap V_{j}: w_{j}=0 .
$$

Then $\mathcal{U}=\left\{U_{i}\right\}$ is a finite covering of $C$ by open sets on which the coordinate charts $z_{i}=\left(z_{i}^{1}, \ldots, z_{i}^{n}\right)$ are defined. Let

$$
z_{k}=\varphi_{k j}\left(z_{j}\right)=\varphi_{k} \varphi_{j}^{-1}\left(z_{j}\right)
$$

be the transition function of $C$ on $U_{k j}:=U_{k} \cap U_{j}$. It is a biholomorphic mapping from $\varphi_{j}\left(U_{k j}\right)$ onto $\varphi_{k}\left(U_{k j}\right)$ in $\mathbf{C}^{n}$. Then $T C$ has a basis

$$
e_{j, \alpha}:=\frac{\partial}{\partial z_{j}^{\alpha}}, \quad 1 \leq \alpha \leq n
$$

over $U_{j}$ and its transition matrices $s_{k j}$ have the form

$$
s_{k j, \beta}^{\alpha}\left(z_{j}\right):=\left.\frac{\partial z_{k}^{\alpha}}{\partial z_{j}^{\beta}}\right|_{U_{j} \cap U_{k}} .
$$

Thus for $\eta_{k}^{\alpha} \frac{\partial}{\partial z_{k}^{\alpha}}=\eta_{j}^{\alpha} \frac{\partial}{\partial z_{j}^{\alpha}}$ on $U_{j} \cap U_{k}$, we have $\eta_{k}=s_{k j}\left(z_{j}\right) \eta_{j}$. As to the normal bundle $N_{C}$, its transition matrices $t_{k j, \nu}^{\mu}\left(z_{j}\right):=\left.\frac{\partial w_{k}^{\mu}}{\partial w_{j}^{\nu}}\right|_{U_{j} \cap U_{k}}$ on $U_{j} \cap U_{k}$ are for the basis

$$
f_{j, \mu}:=\frac{\partial}{\partial w_{j}^{\mu}} \quad \bmod T C, \quad 1 \leq \mu \leq d .
$$

Thus for $\xi_{k}^{\mu} f_{j, \mu}=\xi_{j}^{\mu} f_{k, \mu}$, we have $\xi_{k}=t_{k j}\left(z_{j}\right) \xi_{j}$. With notation $(2.1)$, the transition matrices of $\left.T M\right|_{C}$ are then of the form

$$
g_{k j}:=\left(\begin{array}{cc}
s_{k j} & l_{k j} \\
0 & t_{k j}
\end{array}\right)\left(z_{j}\right) \quad \text { on } U_{j} \cap U_{k}
$$

for some $n \times d$ matrices $l_{j k}$. Note that $\left.\frac{\partial w_{j}}{\partial z_{k}}\right|_{C}=0$.

Throughout the paper, $\tau_{k j}\left(z_{j}\right)$ are the transition matrices of $N_{C}^{*}$ for the base $d w_{j}$. Note that

$$
\tau_{k j}=\left(t_{k j}^{-1}\right)^{t} .
$$

More specifically, if $w_{j, \mu}^{*}:=\left.d w_{j}^{\mu}\right|_{U_{j}}$ and $\zeta_{j}^{\mu} w_{j, \mu}^{*}=\zeta_{k}^{\mu} w_{k, \mu}^{*}$, then (2.5) becomes

$$
\zeta_{k}^{*}=\left(t_{k j}^{-1}\left(z_{j}\right)\right)^{t} \zeta_{j}^{*}, \quad w_{k}^{*}=t_{k j}\left(z_{j}\right) w_{j}^{*}
$$

We remark that the cocycle conditions (2.4) for $N_{C}$ now takes the form

$$
t_{k j}\left(z_{j}\right) t_{j k}\left(z_{k}\right)=\operatorname{Id} \text { on } U_{j} \cap U_{k}, \quad t_{k j}\left(z_{j}\right) t_{j \ell}\left(z_{\ell}\right)=t_{k \ell}\left(z_{\ell}\right) \text { on } U_{j} \cap U_{k} \cap U_{\ell} \text {. }
$$

We say that $T M$ splits on $C$, if there is a (non-canonical) decomposition

$$
\left.T M\right|_{C}=T C \oplus \tilde{N}_{C}, \quad \tilde{N}_{C} \cong N_{C} .
$$

Equivalently, there exists a system of coordinate charts such that on $C$, the transitions matrices of $\left.T M\right|_{C}$ are of the form

$$
g_{k j}=\left(\begin{array}{cc}
s_{k j} & 0 \\
0 & t_{k j}
\end{array}\right)\left(z_{j}\right) \quad \text { on } U_{j} \cap U_{k} .
$$


In other words, $\left.\frac{\partial z_{j}}{\partial w_{k}}\right|_{C}=0$.

Throughout the paper, we assume that $T M$ splits on $C$ and we fix a splitting (2.10). Then the change of bases of the normal bundle $N_{C}$ has a simple form

$$
z_{k}=\varphi_{k j}\left(z_{j}\right), \quad \frac{\partial}{\partial w_{k}^{\nu}}=t_{j k, \nu}^{\mu}\left(z_{k}\right) \frac{\partial}{\partial w_{j}^{\mu}}, \quad \text { on } U_{j} \cap U_{k}
$$

In summary, for a neighborhood of the embedded manifold $C$ in $M$ with splitting $T_{C} M$, we can find a covering $\mathcal{V}=\left\{V_{i}\right\}$, with $\Phi_{j}\left(V_{j}\right)=\tilde{U}_{i} \times \tilde{W}_{i}$, by open sets on $M$ and coordinates $\left(z_{i}, w_{i}\right)$ defined on $V_{i}$. We assume that $U_{j}:=C \cap V_{i}$ is defined by $\left\{w_{i}=0\right\}$. A neighborhood of $C$ will then be described by transition functions on $V_{k j}$ of the form

$$
\begin{aligned}
z_{k} & =\Phi_{k j}^{h}\left(z_{j}, w_{j}\right):=\varphi_{k j}\left(z_{j}\right)+\phi_{k j}^{h}\left(z_{j}, w_{j}\right) \\
w_{k} & =\Phi_{k j}^{v}\left(z_{j}, w_{j}\right):=t_{k j}\left(z_{j}\right) w_{j}+\phi_{k j}^{v}\left(z_{j}, w_{j}\right) .
\end{aligned}
$$

Here, $\phi_{k j}^{h}\left(\right.$ resp. $\left.\phi_{k j}^{v}\right)$ are holomorphic functions of vanishing order $\geq 2$ along $w_{j}=0$ :

$$
\phi_{k j}^{h}\left(z_{j}, w_{j}\right)=O\left(\left|w_{j}\right|^{2}\right), \quad \phi_{k j}^{v}\left(z_{j}, w_{j}\right)=O\left(\left|w_{j}\right|^{2}\right) .
$$

That $\phi_{k j}^{h}$ vanishes at order $\geq 2$ follows from the fact that $\left.T M\right|_{C}$ splits as $T C \oplus N_{C}$ (see above and [30, proposition 2.9]). Define

$$
N_{k j}\left(h_{j}, v_{j}\right):=\left(\varphi_{k j}\left(z_{j}\right), t_{k j}\left(h_{j}\right) v_{j}\right) .
$$

Our goals are to apply changes of coordinates to simplify $\phi_{k j}^{h}, \phi_{k j}^{v}$, or one of them, according to the problem we study.

2.2. The equivalence of transition functions. The germ of neighborhood of an embedded manifold is well-defined. For the formal normalization, we need to introduce (semi) formal charts and formal neighborhoods of an embedded manifold in a (semi) formal manifold.

Definition 2.1. We call $\hat{M}$ an (admissible and splitting) formal neighborhood of $C$ if there are holomorphic coordinate charts $\varphi_{j}$ on $U_{j}$ where $\left\{U_{j}\right\}$ is a covering of $C$ and there are formal power series

$$
\left(z_{j}, w_{j}\right)=\hat{\Phi}_{j}(p, w):=\left(\varphi_{j}(p), t_{j}(p) w\right)+\sum_{|Q| \geq 2} \Phi_{j, Q}(p) w^{Q},
$$

where $\Phi_{j, Q}$ are holomorphic functions in $U_{j}$ and each $t_{j}$ is an invertible holomorphic $d \times d$ matrix on $U_{j}$. Note that the formal transition functions $\hat{\Phi}_{k j}=\hat{\Phi}_{k} \hat{\Phi}_{j}^{-1}$ have the form

$$
\hat{\Phi}_{k j}\left(z_{j}, w_{j}\right)=\left(\varphi_{k j}\left(z_{j}\right), t_{k j}\left(z_{j}\right) w_{j}\right)+\sum_{|Q|>1} \hat{\Phi}_{k j, Q}\left(z_{j}\right) w_{j}^{Q}, \quad z_{j} \in \varphi_{j}\left(U_{j} \cap U_{k}\right) .
$$

(a) When all $\Phi_{j}$ are holomorphic, the formal neighborhood $\hat{M}$ is called the germ of a (holomorphic) neighborhood of $C$.

(b) $\hat{M}$ is called a linear neighborhood of $C$ if additionally

$$
\hat{\Phi}_{k j}\left(z_{j}, v_{j}\right)=\left(\varphi_{k j}\left(z_{j}\right), t_{k j}\left(z_{j}\right) v_{j}\right)
$$


and each $t_{k j}$ is an invertible holomorphic matrix in $U_{k} \cap U_{j}$. The terminology is meaningful since the $\hat{\Phi}_{k j}$ can be realized as the transition functions of a holomorphic vector bundle over $C$, namely the normal bundle of $C$ in $M$.

We are mainly interested in the classification of a neighborhood of $C$ for a given $C$. Therefore, it is reasonable to assume that the local trivialization of $C$ are fixed. In other words, $\varphi_{k j}$ are fixed and we will only consider mappings sending a neighborhood of $C$ into another neighborhood of $C$ that fix $C$ pointwise.

Definition 2.2. We shall say that $N_{C}$ is a flat (resp. unitary flat), if we can find constant (resp. with values in group of unitary matrices $U_{d}$ ) transition functions in a possibly refined covering. If $T_{C} M:=\left.(T M)\right|_{C}$ is holomorphically flat, or flat, i.e. in some coordinates both transition functions $N_{C}$ and $T C$ are constant matrices, then by (2.7)

$$
\varphi_{k j}\left(z_{j}\right)=s_{k j} z_{j}+c_{k j}
$$

where $s_{k j}$ are constant matrices and $c_{k j}$ are constant vectors. Then, the transition functions of a neighborhood of the zero section of the normal bundle, $\hat{\Phi}_{k j}$ as defined in (2.13) read

$$
A_{k j}\left(z_{j}, w_{j}\right):=\left(s_{k j} z_{j}+c_{k j}, t_{k j} w_{j}\right) .
$$

We will use the following notation: When $N_{C}$ is flat, we write its transition matrices $t_{k j}\left(z_{j}\right)$ as $t_{k j}$, indicating that they are independent of $z_{j}$.

Definition 2.3. We shall say that a change of coordinates $\left\{F_{j}\right\}$ preserves the germ of a neighborhood of the zero section of $N_{C}$ with transition maps $\left\{N_{k j}\right\}$ if each $F_{j}$ is biholomorphic and fixes $v_{j}=0$ pointwise and $F_{k} N_{k j}=N_{k j} F_{j}$, in which case we say that $\left\{F_{j}\right\}$ preserves $\left\{N_{k j}\right\}$ for simplicity.

We further observe the following.

Lemma 2.4. Let $M, \hat{M}$ be two (admissible) neighborhoods of $C$, of which coordinate charts are $\left\{\Phi_{j}\right\},\left\{\hat{\Phi}_{j}\right\}$, respectively. Let $\Phi_{k j}=\Phi_{k} \Phi_{j}^{-1}$ and $\hat{\Phi}_{k j}=\hat{\Phi}_{k} \hat{\Phi}_{j}^{-1}$.

(a) There is a biholomorphic mapping $F: M \rightarrow \hat{M}$, defined near $C$ and fixing $C$, if and only if there are biholomorphic mappings $F_{j}$ satisfying

$$
F_{k} \hat{\Phi}_{k j}\left(z_{j}, w_{j}\right)=\Phi_{k j} F_{j}\left(z_{j}, w_{j}\right), \quad F_{j}\left(z_{j}, 0\right)=\left(z_{j}, 0\right) .
$$

(b) If $F_{j}$ satisfies (2.14), then

$$
\begin{gathered}
F_{j}\left(z_{j}, w_{j}\right)=L F_{j}\left(z_{j}, w_{j}\right)+O\left(\left|w_{j}\right|^{2}\right), \quad L F_{j}=\left(z_{j}+s_{j}\left(z_{j}\right) w_{j}, u_{j}\left(z_{j}\right) w_{j}\right), \\
s_{k}\left(\varphi_{k j}\left(z_{j}\right)\right) t_{k j}\left(z_{j}\right)=D \varphi_{k j}\left(z_{j}\right) s_{j}\left(z_{j}\right), \\
u_{k}\left(\varphi_{k j}\left(z_{j}\right)\right) t_{k j}\left(z_{j}\right)=t_{k j}\left(z_{j}\right) u_{j}\left(z_{j}\right) .
\end{gathered}
$$

Assume further that $F$ preserves the splitting. Then $s_{j}=0$.

(c) Let $T C$ and $N_{C}$ be flat and let $F_{j}$ be (semi) formal biholomorphism fixing $C$ pointwise. Suppose that $F_{k}^{-1} \Phi_{k j} F_{j}=N_{k j}+O\left(|v|_{j}^{2}\right)$. Then $\left\{L F_{j}\right\}$ preserves $\left\{N_{k j}\right\}$, i.e. $L F_{k} N_{k j}\left(L F_{j}\right)^{-1}=N_{k j}$, where

$F_{j}\left(h_{j}, v_{j}\right)=L F_{j}\left(h_{j}, v_{j}\right)+O\left(\left|v_{j}\right|^{2}\right), \quad L F_{j}\left(h_{j}, v_{j}\right)=\left(h_{j}+s_{j}\left(h_{j}\right) v_{j}, u_{j}\left(h_{j}\right) v_{j}\right)$. 
Proof. The points $(a),(b)$ can be verified easily. For $(c)$, let us expand $F_{k} \Phi_{k j}\left(h_{j}, v_{j}\right)=$ $N_{k j} \circ F_{j}\left(h_{j}, v_{j}\right)+O\left(\left|v_{j}\right|^{2}\right)$ and compare the constant and linear terms in $v_{j}$. We obtain

$$
\begin{gathered}
\varphi_{k j}\left(h_{j}\right)+s_{j}\left(\varphi_{k j}\left(h_{j}\right)\right) t_{k j} v_{j}=\varphi_{k j}\left(h_{j}+s_{j}\left(h_{j}\right) v_{j}\right)+O\left(\left|v_{j}\right|^{2}\right), \\
u_{k}\left(\varphi_{k j}\left(h_{j}\right)\right) t_{k j} v_{j}=t_{k j} u_{j}\left(h_{j}\right) v_{j}+O\left(\left|v_{j}\right|^{2}\right) .
\end{gathered}
$$

Here we have used the assumption that $t_{k j}$ are constant. Since $\varphi_{k j}$ are affine, the two identities still hold if we drop $O\left(\left|v_{j}\right|^{2}\right)$ from them. This shows that $L F_{k} N_{k j}=N_{k j} L F_{j}$, again using the fact that $t_{k j}$ are constant and $\varphi_{k j}$ are affine.

Finally, we mention that we will choose the atlas of $C$ so that each $\varphi_{j}$ is a biholomorphism from $U_{j}$ onto the unit polydisc $\Delta_{n}$ in $\mathbf{C}^{n}$ and from a neighborhood $\tilde{U}_{j}$ of $\overline{U_{j}}$ onto another larger polydisc. When $C$ is embedded in a complex manifold $M$, we can extend $\varphi_{j}$ to $V_{j}$ to get a coordinate chart $\Phi_{j}$ on $V_{j}$ such that $\Phi_{j}$ maps $V_{j}$ onto $U_{j} \times \Delta_{\delta}^{d}$. This can be achieved since any holomorphic vector bundle over $\tilde{U}_{j}$ is holomorphically trivial. Thus $\left.N_{C}\right|_{U_{j}}$ splits. Consequently, we can use a flow box of holomorphic normal vector fields to construct the required $\Phi_{j}$. Therefore, if $C$ is embedded into another complex manifold $\tilde{M}$, we will choose the atlas of a neighborhood of $C$ in $\tilde{M}$ such that the restriction of the chart on $U_{j}$ agrees with $\varphi_{j}$.

Therefore, we introduce the following.

Definition 2.5. We say that a formal neighborhood $\left\{\Phi_{k j}\right\}$ of $C$ is equivalent to a neighborhood $\left\{\hat{\Phi}_{k j}\right\}$ of $C$ in $M$ by a formal mapping $F$ that is tangent to the identity and preserves the splitting of $T_{C} M$, if there are formal maps $F_{j}\left(z_{j}\right)=\left(z_{j}, w_{j}\right)+\sum_{|Q|>1} F_{j, Q}\left(z_{j}\right) w_{j}^{Q}$ such that $F_{j, Q}\left(z_{j}\right)$ are holomorphic functions in $U_{j}$ and as power series in $w_{j}$

$$
F_{k} \hat{\Phi}_{k j}\left(z_{j}, w_{j}\right)=\Phi_{k j} F_{j}\left(z_{j}, w_{j}\right) .
$$

We take $F=\hat{\Phi}_{j}^{-1} F_{j} \Phi_{j}$, which is well-defined, when $\Phi_{k j}=\Phi_{k} \Phi_{j}^{-1}$ and $\hat{\Phi}_{k j}=\hat{\Phi}_{k} \hat{\Phi}_{j}^{-1}$.

2.3. The full Linearization of a neighborhood. In this case, our goal is to seek new coordinates $\left(h_{k}, v_{k}\right)$ so that all $\phi_{k j}^{h}, \phi_{k j}^{v}$ are 0 .

Let us consider a change of coordinates in a neighborhood of $C$ by modifying the old coordinate charts $\left(z_{k}, w_{k}\right)$ via $F_{k}$. We write it as

$$
\begin{aligned}
F_{k}: & =F_{k}^{h}\left(h_{k}, v_{k}\right):=h_{k}+f_{k}^{h}\left(h_{k}, v_{k}\right), \\
w_{k} & =F_{k}^{v}\left(h_{k}, v_{k}\right):=v_{k}+f_{k}^{v}\left(h_{k}, v_{k}\right) .
\end{aligned}
$$

Here, $f_{k}^{h}\left(h_{k}, v_{k}\right)$ and $f_{k}^{v}\left(h_{k}, v_{k}\right)$ are holomorphic functions vanishing at order $\geq 2$ at $v_{k}=0$. In particular, $C$ is pointwise fixed by the change as $z_{k}=h_{k}$ on $C$ (i.e. for $v_{k}=0$ ). We require that the inverse of $F_{k}$ is defined in a possibly smaller open sets $\hat{V}_{k} \subset \varphi_{k}\left(U_{k}\right)$ such that the union of $\Phi_{k}^{-1}\left(\hat{V}_{k}\right)$ remains a neighborhood of $C$ in $M$.

We recall that the cocyle condition (2.9) on the transition matrices $t_{k j}$ has the form

$$
\begin{aligned}
t_{k j}\left(z_{j}\right) t_{j k}\left(\varphi_{k j}\left(z_{j}\right)\right) & =\mathrm{Id}, \\
t_{k j}\left(\varphi_{j \ell}\left(z_{\ell}\right)\right) t_{j \ell}\left(z_{\ell}\right) & =t_{k \ell}\left(z_{\ell}\right) .
\end{aligned}
$$

Let us assume that the (a priori formal) change of coordinates (2.15), maps a neighborhood $C$ to a neighborhood of the zero section in the normal bundle. This means that, in 
these new coordinates, we have

$$
N_{k j}:=F_{k}^{-1} \Phi_{k j} F_{j}: \begin{aligned}
h_{k} & =\varphi_{k j}\left(h_{j}\right) \\
v_{k} & =t_{k j}\left(z_{j}\right) v_{j}
\end{aligned}
$$

Let us write down the above "conjugacy equations". We first consider the horizontal equation of

$$
F_{k} N_{k j}=\Phi_{k j} F_{j}
$$

On the left side of the equation, we have

$$
z_{k}=h_{k}+f_{k}^{h}\left(h_{k}, v_{k}\right)=\varphi_{k j}\left(h_{j}\right)+f_{k}^{h}\left(\varphi_{k j}\left(h_{j}\right), t_{k j}\left(h_{j}\right) v_{j}\right)
$$

On the other side, we have

$$
z_{k}=\varphi_{k j}\left(h_{j}+f_{j}^{h}\left(h_{j}, v_{j}\right)\right)+\phi_{k j}^{h}\left(h_{j}+f_{j}^{h}, v_{j}+f_{j}^{v}\right) .
$$

Let us define the horizontal cohomological operator to be

$$
\mathcal{L}_{k j}^{h}\left(f_{j}^{h}\right):=f_{k}^{h}\left(\varphi_{k j}\left(h_{j}\right), t_{k j}\left(h_{j}\right) v_{j}\right)-s_{k j}\left(h_{j}\right) f_{j}^{h}\left(h_{j}, v_{j}\right)
$$

Recall that $s_{k j}\left(h_{j}\right)=D \varphi_{k j}\left(h_{j}\right)$ is the Jacobian matrix of $\varphi_{k j}$. Hence, we can write the previous horizontal equation as

$$
\begin{aligned}
\mathcal{L}_{k j}^{h}\left(f_{j}^{h}\right)= & \phi_{k j}^{h}\left(h_{j}+f_{j}^{h}, v_{j}+f_{j}^{v}\right) \\
& +\varphi_{k j}\left(h_{j}+f_{j}^{h}\left(h_{j}, v_{j}\right)\right)-\varphi_{k j}\left(h_{j}\right)-D \varphi_{k j}\left(h_{j}\right) f_{j}^{h}\left(h_{j}, v_{j}\right) .
\end{aligned}
$$

Let us consider the vertical equation. We have, on one side of the equation,

$$
w_{k}=v_{k}+f_{k}^{v}\left(h_{k}, v_{k}\right)=t_{k j}\left(h_{j}\right) v_{j}+f_{k}^{v}\left(\varphi_{k j}\left(h_{j}\right), t_{k j}\left(h_{j}\right) v_{j}\right) .
$$

On the other side, we have

$$
w_{k}=t_{k j}\left(h_{j}+f_{j}^{h}\right)\left(v_{j}+f_{j}^{v}\right)+\phi_{k j}^{v}\left(h_{j}+f_{j}^{h}, v_{j}+f_{j}^{v}\right) .
$$

Let us define the vertical cohomological operator to be

$$
\mathcal{L}_{k j}^{v}\left(f_{j}^{v}\right):=f_{k}^{v}\left(\varphi_{k j}\left(h_{j}\right), t_{k j}\left(h_{j}\right) v_{j}\right)-t_{k j}\left(h_{j}\right) f_{j}^{v} .
$$

Hence, we can write the previous vertical equation as

$$
\begin{aligned}
\mathcal{L}_{k j}^{v}\left(f_{j}^{v}\right)= & \phi_{k j}^{v}\left(h_{j}+f_{j}^{h}, v_{j}+f_{j}^{v}\right) \\
& +\left(t_{k j}\left(h_{j}+f_{j}^{h}\left(h_{j}, v_{j}\right)\right)-t_{k j}\left(h_{j}\right)\right) f_{j}^{v} \\
& +\left(t_{k j}\left(h_{j}+f_{j}^{h}\left(h_{j}, v_{j}\right)\right)-t_{k j}\left(h_{j}\right)\right) v_{j} .
\end{aligned}
$$

2.4. Horizontal foliations and vertical trivializations. Let us assume that there exists a non singular holomorphic foliation having $C$ as a leaf. We seek holomorphic functions $f_{j}=\left(f_{j, 1}, \ldots, f_{j, d}\right)$ defined in a neighborhood $V_{j}$ of $U_{j}$ such that $f_{j}=0$ on $U_{j}$ and $d f_{j, 1} \wedge \cdots \wedge$ $d f_{j, d} \neq 0$. Then, we may use $\left(h_{j}, v_{j}\right)=\left(z_{j}, f_{j}\left(z_{j}, w_{j}\right)\right)$ as a coordinate mapping on $V_{j}$, which changes variables in vertical components. We then prove that in these new coordinates, the transition functions of a neighborhood of $C$ are of the form $\hat{\Phi}_{k j}=\left(\hat{\Phi}_{k j}^{h}, \hat{\Phi}_{k j}^{v}\right)$ such that $\Phi_{k j}^{v}$ are independent of $h_{j}$. We remark that $N_{C}$ must be flat if a horizontal foliation exists. 
Proposition 2.6. Assume that there is smooth holomorphic horizontal foliation defined in a neighborhood $V$ of $C$ in $M$. By a refinement of $U_{j}$, then there exists a change of variables of the form

$$
z_{k}=h_{k} \quad w_{k}=s\left(h_{j}\right) v_{j}+O\left(\left|v_{j}\right|^{2}\right)
$$

so that in the new variables, we have

$$
\begin{aligned}
h_{k} & =\varphi_{k j}\left(h_{j}\right)+\phi_{k j}^{h}\left(h_{j}, v_{j}\right), \\
v_{k} & =\tilde{t}_{k j} v_{j}+\sum_{|Q|>1} c_{k j, Q} v_{j}^{Q},
\end{aligned}
$$

where $\tilde{t}_{k j}, c_{k j, Q}$ are constants.

Proof. By a refinement, we may assume that the foliation on $V_{j}$ is given $W_{j}\left(h_{j}, v_{j}\right)=c s t$ by holomorphic functions $W_{j}=\left(W_{j, 1}, \ldots, W_{j, d}\right)$ such that $W_{j}=0$ on $U_{j}$ and $d W_{j, 1} \wedge$ $\cdots \wedge d W_{j, d} \neq 0$. We have $W_{k}=\tilde{\Phi}_{k j}^{v} W_{j}$, where $\tilde{\Phi}_{k j}^{v}$ is a biholomorphism of $\left(\mathbb{C}^{d}, 0\right)$ with $\tilde{\Phi}_{k j}^{v}(0)=0$. Then $\tilde{W}_{j}=\left(z_{j}, W_{j}\right)$ is a biholomorphism defined on $V_{j}$ and fixing $C \cap V_{j}$ pointwise, by shrinking $V_{j}$ if necessary in the vertical direction. Since $\tilde{W}_{j}$ is invertible, we can define $\tilde{\Phi}_{k j}^{h}=z_{k} \tilde{W}_{j}^{-1}$ Then we have $\tilde{\Phi}_{k j}^{h} \tilde{W}_{j}=z_{k}$. Therefore,

$$
\tilde{W}_{k} \tilde{W}_{j}^{-1}\left(h_{j}, v_{j}\right)=\left(\tilde{\Phi}_{k j}^{h}\left(h_{j}, v_{j}\right), \tilde{\Phi}_{k j}^{v}\left(v_{j}\right)\right) .
$$

Set $F_{j}=\Phi_{j} \tilde{W}_{j}^{-1}$. We have $F_{j}^{h}\left(h_{j}, v_{j}\right)=h_{j}$. We now get

$$
F_{k}^{-1} \Phi_{k} \Phi_{j}^{-1} F_{j}=\tilde{W}_{k} \tilde{W}_{j}^{-1}=\tilde{\Phi}_{k j}
$$

In this paper, we will approach the horizontal foliation problem via the following vertical linearization when $N_{C}$ is unitary.

2.5. The vertical linearization. Here we seek new coordinates $\left(h_{j}, v_{j}\right)$ from $\left(z_{j}, w_{j}\right)$ such that the vertical component of the new $\Phi_{k j}$ agrees with the vertical component of $N_{k j}$. In Lemma 2.17 we will show that if such formal coordinates exist, then the vertical linearization can be achieved by changing vertical coordinates only, i.e. a coordinate change of the form

$$
w_{k}=F_{k}^{v}\left(h_{k}, v_{k}\right):=v_{k}+f_{k}^{v}\left(h_{k}, v_{k}\right), \quad z_{k}=h_{k}
$$

For the vertical linearization, we only need to consider the vertical part of transition functions so that in the new variables, we have

$$
\begin{aligned}
h_{k} & =\hat{\Phi}_{k j}^{h}\left(h_{j}, v_{j}\right):=\varphi_{k j}\left(h_{j}\right)+\hat{\phi}_{k j}^{h}\left(h_{j}, v_{j}\right) \\
v_{k} & =t_{k j}\left(h_{j}\right) v_{j} .
\end{aligned}
$$

Here, $\hat{\phi}_{k j}^{h}\left(h_{j}, v_{j}\right)$ vanishes up to order 2 at $v_{j}=0$. The vertical equation reads

$$
t_{k j}\left(h_{j}\right)\left(v_{j}+f_{j}^{v}\right)+\phi_{k j}^{v}\left(h_{j}, v_{j}+f_{j}^{v}\right)=w_{k}=t_{k j}\left(h_{j}\right) v_{j}+f_{k}^{v}\left(\hat{\Phi}_{k j}^{h}\left(h_{j}, v_{j}\right), t_{k j}\left(h_{j}\right) v_{j}\right) .
$$

Using the previous notation, we finally obtain the following "conjugacy equations"

$$
\mathcal{L}_{k j}^{v}\left(f_{j}^{v}\right)=\phi_{k j}^{v}\left(h_{j}, v_{j}+f_{j}^{v}\right)-\left(f_{k}^{v}\left(\hat{\Phi}_{k j}^{h}\left(h_{j}, v_{j}\right), t_{k j}\left(h_{j}\right) v_{j}\right)-f_{k}^{v}\left(\varphi_{k j}\left(h_{j}\right), t_{k j}\left(h_{j}\right) v_{j}\right)\right) .
$$


Having determined the coordinate change, let us find the horizontal component $\hat{\phi}_{k j}^{h}$ from the horizontal equation

$$
\varphi_{k j}\left(h_{j}\right)+\phi_{k j}^{h}\left(h_{j}, v_{j}+f_{j}^{v}\right)=z_{k}=\hat{\Phi}_{k j}^{h}\left(h_{j}, v_{j}\right)=\varphi_{k j}\left(h_{j}\right)+\hat{\phi}_{k j}^{h}\left(h_{j}, v_{j}\right) .
$$

We get

$$
\hat{\phi}_{k j}^{h}\left(h_{j}, v_{j}\right)=\phi_{k j}^{h}\left(h_{j}, v_{j}+f_{j}^{v}\right)
$$

2.6. Coboundary operators in symmetric powers and coordinates. In this subsection, we establish the connections between coordinate changes and formal obstructions to the full linearization and vertical linearization via cohomological groups. In local dynamics, the resonant terms play an important role in the construction of normal forms at least at the formal level, while non-resonant terms play another important role in coordinate changes. In all problems, obstructions are described via the first cohomological groups, while the coordinate changes are described via solutions to the cohomological equations of first order approximation.

Let $E^{\prime}$ be a vector bundle of rank $\tau$ over $C$. Let $\mathcal{U}=\left\{U_{i}\right\}$ be a covering of $C$ as above. Let $e_{j}:=\left\{e_{j, 1}, \ldots, e_{j, \tau}\right\}$ be a basis over $U_{j}$ and let $\xi_{j}:=\left(\xi_{j}^{1}, \ldots, \xi_{j}^{\tau}\right)^{t}$ be coordinates in $e_{j}$. Let $s_{k j}\left(z_{j}\right)$ be the transition matrices of $E^{\prime}$ over $U_{k} \cap U_{j}$. Using notation in (2.3), we have

$$
\begin{gathered}
\xi_{k}^{\alpha}=s_{k j, \beta}^{\alpha}\left(z_{j}\right) \xi_{j}^{\beta}, \quad e_{k ; \alpha}=s_{j k, \alpha}^{\beta}\left(z_{k}\right) e_{j, \beta}, \\
z_{k}=\varphi_{k j}\left(z_{j}\right), \quad \xi_{k}=s_{k j}\left(z_{j}\right) \xi_{j}, \quad e_{k}=\left(s_{k j}^{-1}\left(z_{j}\right)\right)^{t} e_{j},
\end{gathered}
$$

where $\varphi_{k j}$ are the transition functions of $C$. For $N_{C}^{*}$, by (2.8) we have

$$
\zeta_{k}=\left(t_{k j}^{-1}\right)^{t}\left(z_{j}\right) \zeta_{j}, \quad w_{k}^{*}=t_{k j}\left(z_{j}\right) w_{j}^{*}, \quad z_{k}=\varphi_{k j}\left(z_{j}\right)
$$

The following fact is well-known. We provide a proof for the reader's convenience. Let us first introduce

$$
\tilde{f}_{i_{0} \cdots i_{q}}^{\lambda}\left(z_{i_{q}}, \zeta_{i_{q}}\right):=\sum_{|Q|=L} f_{i_{0} \cdots i_{q} ; Q}^{\lambda}\left(z_{i_{q}}\right) \zeta_{i_{q}}^{Q},
$$

for a cochain $\left\{f_{I}\right\} \in C^{q}\left(\left\{U_{j}\right\}, \mathcal{O}\left(E \otimes S^{L}\left(N_{C}^{*}\right)\right)\right)$ given by

$$
f_{i_{0} \cdots i_{q}}(p)=\sum_{\lambda=1}^{\tau} \sum_{|Q|=L} f_{i_{0} \cdots i_{q} ; Q}^{\lambda}\left(z_{i_{q}}(p)\right) e_{i_{0}, \lambda}(p) \otimes\left(w_{i_{q}}^{*}(p)\right)^{Q}
$$

where each $f_{i_{0} \ldots i_{q} ; Q}^{\lambda}$ is a holomorphic function on $\varphi_{i_{q}}\left(U_{i_{0} \cdots i_{q}}\right)$, and $U_{i_{0} \cdots i_{q}}$ denotes as usual $U_{i_{0}} \cap \cdots \cap U_{i_{q}}$. Here we have chosen a representation of cochains in bases that arise from the linearized equations for the problems described above. 
Let $f_{i_{0} \cdots \hat{i}_{\ell} \cdots i_{q+1}}$ denote $f_{i_{0} \cdots i_{\ell-1} i_{\ell+1} \cdots i_{q+1}}$. Then $(\delta f)_{i_{0} \cdots i_{q+1}}=\sum(-1)^{\ell} f_{i_{0} \cdots \hat{i}_{\ell} \cdots i_{q+1}}$ becomes

$$
\begin{aligned}
(\delta f)_{i_{0} \cdots i_{q+1}}= & \sum_{\ell=1}^{q}(-1)^{\ell} \sum_{\lambda=1}^{\tau} \sum_{|Q|=L} f_{i_{0} \cdots \hat{i}_{\ell} \cdots i_{q+1} ; Q}^{\lambda}\left(z_{i_{q+1}}(p)\right) e_{i_{0}, \lambda}(p) \otimes\left(w_{i_{q+1}}^{*}(p)\right)^{Q} \\
& +\sum_{\lambda=1}^{\tau} \sum_{|Q|=L} f_{i_{1} \cdots i_{q+1} ; Q}^{\lambda}\left(z_{i_{q+1}}(p)\right) e_{i_{1}, \lambda}(p) \otimes\left(w_{i_{q+1}}^{*}(p)\right)^{Q} \\
& -(-1)^{q} \sum_{\lambda=1}^{\tau} \sum_{|Q|=L} f_{i_{0} \cdots i_{q} ; Q}^{\lambda}\left(z_{i_{q}}(p)\right) e_{i_{0}, \lambda}(p) \otimes\left(w_{i_{q}}^{*}(p)\right)^{Q} \\
= & : \sum_{\lambda=1}^{\tau} \sum_{|Q|=L} g_{i_{0} \cdots i_{q+1}}^{\lambda}\left(z_{q+1}\right) e_{i_{0}, \lambda}(p) \otimes\left(w_{i_{q+1}}^{*}(p)\right)^{Q} .
\end{aligned}
$$

By (2.22), we have $e_{i_{1}, \lambda}=s_{i_{0} i_{1}, \lambda}^{\mu} e_{i_{0}, \mu}$. In notation (2.24), we can express

$$
\begin{aligned}
\tilde{g}_{i_{0} \cdots i_{q+1}}^{\lambda}\left(z_{i_{q+1}}, \zeta_{i_{q+1}}\right)= & \sum_{\ell=1}^{q}(-1)^{\ell} \tilde{f}_{i_{0} \cdots \hat{i}_{\ell} \cdots i_{q+1}}^{\lambda}\left(z_{i_{q+1}}, \zeta_{i_{q+1}}\right) \\
& +s_{i_{0} i_{1}, \mu}^{\lambda}\left(\varphi_{i_{1} i_{q+1}}\left(z_{q+1}\right)\right) \tilde{f}_{i_{1} \cdots i_{q+1}}^{\mu}\left(z_{i_{q+1}}, \zeta_{i_{q+1}}\right) \\
& \left.-(-1)^{q} f_{i_{0} \cdots i_{q}}^{\lambda}\left(\varphi_{i_{q} i_{q+1}}\left(z_{i_{q+1}}\right), t_{i_{q} i_{q+1}}\left(z_{i_{q+1}}\right) \zeta_{i_{q+1}}\right)\right) .
\end{aligned}
$$

The above computation especially gives us the following formulae for 0 and 1-cochains.

Lemma 2.7. Let $\left\{U_{j}\right\}$ be an open covering of $C$. Let $t_{k j}$ be the transition matrices for $N_{C}$ with respect to basis $w_{j}$ and let $s_{k j}$ be the transitions functions of $E$ with respect to base $e_{j}$. Let

$$
\begin{aligned}
f_{i j}(p) & =\sum_{\lambda=1}^{d} \sum_{|Q|=L} f_{i j ; Q}^{\lambda}\left(z_{j}(p)\right) e_{i, \lambda}(p) \otimes\left(w_{j}^{*}(p)\right)^{Q}, \quad \tilde{f}_{i j}^{\lambda}\left(z_{j}, \zeta_{j}\right):=\sum_{|Q|=L} f_{i j ; Q}^{\lambda}\left(z_{j}\right) \zeta_{j}^{Q}, \\
u_{j}(p) & =\sum_{\lambda=1}^{d} \sum_{|Q|=L} u_{j, Q}^{\lambda}\left(z_{j}(p)\right) e_{j, \lambda}(p) \otimes\left(w_{j}^{*}(p)\right)^{Q}, \quad \tilde{u}_{j}^{\lambda}\left(z_{j}, \zeta_{j}\right):=\sum_{|Q|=L} u_{j ; Q}^{\lambda}\left(z_{j}\right) \zeta_{j}^{Q} .
\end{aligned}
$$

The following hold:

(a) $f:=\left\{f_{i j}\right\} \in Z^{1}\left(\mathcal{U}, \mathcal{O}\left(E \otimes S^{L}\left(N_{C}^{*}\right)\right)\right)$ if and only if

$$
\tilde{f}_{i j}^{\lambda}\left(\varphi_{j k}\left(z_{k}\right), t_{j k}\left(z_{k}\right) \zeta_{k}\right)-\tilde{f}_{i k}^{\lambda}\left(z_{k}, \zeta_{k}\right)+s_{i j, \ell}^{\lambda}\left(z_{j}\right) \tilde{f}_{j k}^{\lambda}\left(z_{k}, \zeta_{k}\right)=0 .
$$

(b) $u:=\left\{u_{j}\right\}$ solves the first order cohomological equation $\delta u=f$ if and only if

$$
s_{i j, \ell}^{\lambda}\left(z_{j}\right) \tilde{u}_{j}^{\ell}\left(z_{j}, \zeta_{j}\right)-\tilde{u}_{i}^{\lambda}\left(\varphi_{i j}\left(z_{j}\right), t_{i j}\left(z_{j}\right) \zeta_{j}\right)=\tilde{f}_{i j}^{\lambda}\left(z_{j}, \zeta_{j}\right) .
$$

We notice that according to (2.16) and (2.18), we have

$$
-\mathcal{L}(f)=-\left(\mathcal{L}^{h}\left(f^{h}\right), \mathcal{L}^{v}\left(f^{v}\right)\right)=\delta(f):=\left(\delta^{h}\left(f^{h}\right), \delta^{v}\left(f^{v}\right)\right) .
$$


2.7. Formal obstructions in cohomology groups. Recall that

$$
N_{k j}\left(h_{j}, v_{j}\right):=\left(\varphi_{k j}\left(z_{j}\right), t_{k j}\left(h_{j}\right) v_{j}\right) .
$$

Let us denote the properties depending on an order $m \geq 1$ :

$\left(L_{m}(\mathcal{U})\right)$ : the neighborhood of $C$ matches the neighborhood of zero section of the normal bundle up to order $m$.

$\left(V_{m}(\mathcal{U})\right)$ : the vertical components of the transition functions of neighborhoods of $C$ in $M$ and in $N_{C}$ match up to order $m$.

That embedding of $C$ has property $\left(L_{m}\right)$ (resp. $\left(V_{m}\right)$ ) means that the order along $v_{j}=0$ of $\left(\phi_{k j}^{h}\left(h_{j}, v_{j}\right), \phi_{k j}^{v}\left(h_{j}, v_{j}\right)\right)$ (resp. $\left.\phi_{k j}^{v}\left(h_{j}, v_{j}\right)\right)$ as defined in $(2.11)$ is $\geq m+1$.

Definition 2.8. We shall say that $N_{C}$ is a flat (resp. unitary flat), if we can find constant (resp. with values in group of unitary matrices $U_{d}$ ) transition functions in a possibly refined covering.

We will use the following notation: When $N_{C}$ is flat, we write its transition matrices $t_{k j}\left(z_{j}\right)$ as $t_{k j}$, indicating that they are independent of $z_{j}$.

Definition 2.9. We shall say that a change of coordinates $\left\{F_{j}\right\}$ preserves the germ of a neighborhood of the zero section of $N_{C}$ with transition maps $\left\{N_{k j}\right\}$ if $F_{k} N_{k j}=N_{k j} F_{j}$, in which case we says that $\left\{F_{j}\right\}$ preserves $\left\{N_{k j}\right\}$ for simplicity.

Lemma 2.10. Let the transition functions $\Phi_{k j}$ of a neighborhood of $C$ be given by (2.11)$(2.12)$.

(a) Assume that $C$ satisfies $L_{m}$. Then the horizontal and vertical components satisfy

$$
\begin{array}{ll}
{\left[\phi_{k j}^{h}\right]^{\ell} \in Z^{1}\left(\mathcal{U}, T C \otimes S^{\ell}\left(N_{C}^{*}\right)\right),} & \text { if } m<\ell \leq 2 m ; \\
{\left[\phi_{k j}^{v}\right]^{\ell} \in Z^{1}\left(\mathcal{U}, N_{C} \otimes S^{\ell}\left(N_{C}^{*}\right)\right),} & \text { if } \ell=m+1 .
\end{array}
$$

If $N_{C}$ is flat, then the vertical component of $\Phi_{k j}$ further satisfies

$$
\left[\phi_{k j}^{v}\right]^{\ell} \in Z^{1}\left(\mathcal{U}, N_{C} \otimes S^{\ell}\left(N_{C}^{*}\right)\right), \quad m+1<\ell \leq 2 m .
$$

(b) Let $C$ satisfy $V_{m}$. Assume that $N_{C}$ is flat. Then

$$
\left[\phi_{k j}^{v}\right]^{\ell} \in Z^{1}\left(\mathcal{U}, N_{C} \otimes S^{\ell}\left(N_{C}^{*}\right)\right), \quad \ell=m+1 .
$$

Proof. When $\ell=m+1,(a)$ is in Griffiths [15], Morrow-Rossi [30] and (b) is proved in Ueda [40] for flat line bundle $N_{C}^{*}$ over a compact curve $C$.

(a) The general case can be verified by using Lemma 2.7 to compare coefficients of $w_{j}^{\alpha}$ on both sides of $\Phi_{i j}\left(z_{j}, w_{j}\right)=\Phi_{i k} \circ \Phi_{k j}\left(z_{j}, w_{j}\right)$ for $|\alpha| \leq 2 m$. Indeed, we have $\Phi_{i k}=$ $N_{i k}+\left(\phi_{i k}^{h}, \phi_{i k}^{v}\right)$ and $\left(\phi_{i k}^{h}, \phi_{i k}^{v}\right)\left(z_{k}, w_{k}\right)=O\left(\left|w_{k}\right|^{m+1}\right)$ with $m \geq 1$. Thus

$$
\begin{aligned}
N_{i k} \circ \Phi_{k j}\left(z_{j}, w_{j}\right)= & \left\{N_{i k} \circ N_{k j}+D N_{i k} \circ N_{k j} \cdot\left(\phi_{k j}^{h}, \phi_{k j}^{v}\right)\right\}\left(z_{j}, w_{j}\right)+O\left(\left|w_{j}\right|^{2 m+1}\right) \\
= & N_{i k} \circ N_{k j}\left(z_{j}, w_{j}\right)+\left(s_{i k}\left(\varphi_{k j}\left(z_{j}\right)\right) \phi_{k j}^{h}, t_{i k}\left(\varphi_{k j}\left(z_{j}\right)\right) \phi_{k j}^{v}\right) \\
& +\left(0, D t_{i k}\left(\varphi_{k j}\left(z_{j}\right)\right) \phi_{k j}^{h}\left(z_{j}\right) t_{k j}\left(z_{j}\right) w_{j}\right)+O\left(\left|w_{j}\right|^{2 m+1}\right) .
\end{aligned}
$$


Here $s_{k j}$ are the transition matrices of $T C$ given by (2.7). Therefore,

$$
\begin{aligned}
\Phi_{i k} \circ \Phi_{k j}\left(z_{j}, w_{j}\right)= & N_{i k} \circ \Phi_{k j}\left(z_{j}, w_{j}\right)+\left(\phi_{i k}^{h}, \phi_{i k}^{v}\right) \circ \Phi_{k j}\left(z_{j}, w_{j}\right) \\
= & \left\{N_{i k} \circ N_{k j}+\left(\phi_{i k}^{h}, \phi_{i k}^{v}\right) \circ N_{k j}\right\}\left(z_{j}, w_{j}\right) \\
& +\left(s_{i k}\left(\varphi_{k j}\left(z_{j}\right)\right) \phi_{k j}^{h}\left(z_{j}, w_{j}\right), t_{i k}\left(\varphi_{k j}\left(z_{j}\right)\right) \phi_{k j}^{v}\left(z_{j}, w_{j}\right)\right) \\
& +\left(0, D t_{i k}\left(\varphi_{k j}\left(z_{j}\right)\right) \phi_{k j}^{h}\left(z_{j}\right) t_{k j}\left(z_{j}\right) w_{j}\right)+O\left(\left|w_{j}\right|^{2 m+1}\right) .
\end{aligned}
$$

Comparing both sides of $\Phi_{i j}\left(z_{j}, w_{j}\right)=\Phi_{i k} \circ \Phi_{k j}\left(z_{j}, w_{j}\right)$ for the coefficients in $w_{j}$ of order $\ell=m+1$, we obtain the desired conclusion by Lemma 2.7.

(b) We have $\Phi_{k j}\left(z_{j}, w_{j}\right)=\left(\varphi_{k j}\left(z_{j}\right)+\phi_{k j}^{h}\left(z_{j}, w_{j}\right), t_{k j} w_{j}+\phi_{k j}^{v}\left(z_{j}, w_{j}\right)\right)$ with $\phi_{k j}^{v}\left(z_{j}, w_{j}\right)=$ $O\left(\left|w_{j}\right|^{m+1}\right)$. Here $t_{k j}$ are constant. We get from the vertical components of $\Phi_{k j}=\Phi_{k i} \Phi_{i j}$ that

$$
\begin{aligned}
\phi_{k j}^{v}\left(z_{j}, w_{j}\right) & =t_{k i} \phi_{i j}^{v}\left(z_{j}, w_{j}\right)+\phi_{k i}^{v}\left(\Phi_{i j}\left(z_{j}, w_{j}\right)\right) \\
& =t_{k i} \phi_{i j}^{v}\left(z_{j}, w_{j}\right)+\varphi_{k i}\left(N_{i j}\left(z_{j}, w_{j}\right)\right)+O\left(\left|w_{j}\right|^{m+2}\right),
\end{aligned}
$$

since $\left(\Phi_{i j}-N_{i j}\right)\left(z_{j}, w_{j}\right)=O\left(\left|w_{j}\right|^{2}\right)$. This shows that $\left\{\left[\phi_{k j}^{v}\right]^{\ell}\right\} \in Z^{1}\left(\mathcal{U}, N_{C} \otimes N_{C}^{* \ell}\right)$ for $\ell=m+1$ by Lemma $2.7(a)$. This gives us (2.27).

2.8. Automorphisms of neighborhood of the zero section of flat vector bundles. Let $\phi_{k j}$ defined on $U_{k} \cap U_{j}$ be the transition functions of $C$. Let $\Phi_{k j}$, defined on $V_{k} \cap V_{j}$, be the transition functions of $M$, and let $N_{k j}$, defined on $\tilde{V}_{k} \cap \tilde{V}_{j}$ be the transition functions of $N_{C}$, with $\tilde{V}_{k}=\pi^{-1} U_{k}$. We identify $\left(C, U_{j}\right)$ as subsets of $\tilde{V}_{j}$ via the zero-section. Recall $\Phi_{k j}, N_{k j}$, and $\phi_{k j}$ are the same on $U_{k} \cap U_{j}$. By Cartan-Serre theorem, for any integer $m$, the space of global sections, $H^{0}\left(C, T_{C} M \otimes S^{m} N_{C}^{*}\right)$, is finite dimensional.

We say that a vector bundle is flat if its transition matrices are locally constant.

Definition 2.11. (1) A formal tangent vector field $Y_{j}$ on $\tilde{V}_{j}$ vanishing at $U_{j}$ is identified with $Y_{j}=\sum_{\ell \geq 1} Y_{j}^{\ell}$ with $Y_{j}^{\ell} \in \Gamma\left(U_{j}, T_{C} M \otimes S^{\ell} N_{C}^{*}\right)$ via

$$
\sum_{|Q|=\ell} a_{Q}^{\alpha}\left(h_{j}\right) v_{j}^{Q} \frac{\partial}{\partial h_{j}^{\alpha}}+b_{Q}^{\lambda}\left(h_{j}\right) v_{j}^{Q} \frac{\partial}{\partial v_{j}^{\lambda}} \mapsto \sum_{|Q|=\ell} a_{Q}^{\alpha}\left(z_{j}\right)\left(w_{j}^{*}\right)^{Q} \frac{\partial}{\partial z_{j}^{\alpha}}+b_{Q}^{\lambda}\left(z_{j}\right)\left(w_{j}^{*}\right)^{Q} \frac{\partial}{\partial w_{j}^{\lambda}} .
$$

Here $\left(h_{j}, v_{j}\right)$ is the coordinate map for $v_{j}^{\lambda} \frac{\partial}{\partial w_{j}^{\lambda}} \in\left(N_{C}\right)_{p}$ and we identity $h_{j}$ with $\left.z_{j}\right|_{U_{j}}$ and $\frac{\partial}{\partial v_{j}}$ with $\left.\frac{\partial}{\partial w_{j}}\right|_{U_{j}}$.

(2) A formal automorphism of $\tilde{V}_{j}$ at $U_{j}$ that is tangent to the identity is an automorphism of a formal neighborhood of the 0 -section of $\tilde{V}_{j}$, fixing $U_{j}$ pointwise.

Lemma 2.12. Let $\left\{F_{j}\right\}_{j}$ be a collection of formal automorphisms of $\tilde{V}_{j}$ fixing $U_{j}$ pointwise. Let $\left\{Y_{j}\right\}_{j}$ be a collection of formal tangent vector fields of $\tilde{V}_{j}$ vanishing at $U_{j}$. We have

(1) $\left\{F_{j}\right\}_{j}$ defines an automorphism $F$ of a formal neighborhood of the 0 -section in $N_{C}$ if and only $F_{k} \circ N_{k j}=N_{k j} \circ F_{j}$ for all $k, j$

(2) Suppose that $N_{C}$ is flat. $\left\{Y_{j}\right\}_{j}$ defines a vector field $Y$ on a formal neighborhood of the 0-section in $N_{C}$ if and only if $\left\{Y_{j}^{\ell}\right\} \in H^{0}\left(C, T_{C} M \otimes S^{\ell} N_{C}^{*}\right)$ for all $\ell$. 
(3) Suppose that $N_{C}$ is not flat. $\left\{Y_{j}\right\}_{j}$ defines a vector field on a formal neighborhood of the 0-section in $N_{C}$ if and only if $\left\{Y_{j}\right\} \in H_{\text {twisted }}^{0}\left(C, T_{C} M \otimes \oplus_{\ell \geq 2} S^{\ell} N_{C}^{*}\right)$ with respect to the linear operator $\delta_{n f}\left(\left\{\left(Y_{j}^{h}, Y_{j}^{v}\right)\right\}\right)=\left\{\left(\tilde{Y}_{k j}^{h}, \tilde{Y}_{k j}^{v}\right)\right\}$ with

$$
\begin{gathered}
\tilde{Y}_{k j}^{h}=Y_{k}^{h}\left(N_{k j}\left(h_{j}, v_{j}\right)\right)-D \phi_{k j}\left(h_{j}\right) Y_{j}^{h}\left(h_{j}, v_{j}\right), \\
\tilde{Y}_{k j}^{v}=Y_{k}^{v}\left(N_{k j}\left(h_{j}, v_{j}\right)\right)-t_{k j}\left(h_{j}\right) Y_{j}^{v}\left(h_{j}, v_{j}\right)-D t_{k j}\left(h_{j}\right) v_{j} \cdot Y_{j}^{h}\left(h_{j}, v_{j}\right) .
\end{gathered}
$$

Proof. Let $\left(h_{j}, v_{j}\right)$ be the coordinates in $N_{C}$ over $U_{j}$. Note that $\left\{Y_{j}\right\}$ defines a global tangent vector filed of $N_{C}$ if and only if $D N_{k j}\left(Y_{j}\right)=Y_{k}$. A homogeneous vector field of degree $\ell$ on $\tilde{V}_{j}$ is an element $Y_{j}^{\ell} \in C^{0}\left(U_{j}, T_{C} M \otimes S^{\ell} N_{C}^{*}\right)$ defined by

$$
Y_{j}^{\ell}\left(h_{j}, v_{j}\right)=\sum_{m=1}^{n} Y_{j, m}^{\ell, h}\left(h_{j}, v_{j}\right) \frac{\partial}{\partial h_{j, m}}+\sum_{r=1}^{d} Y_{j, r}^{\ell, v}\left(h_{j}, v_{j}\right) \frac{\partial}{\partial v_{j, r}}=: Y_{j}^{\ell, h}+Y_{j}^{\ell, v} .
$$

Recall that $N_{k j}\left(h_{j}, v_{j}\right)=\left(\phi_{k j}\left(h_{j}\right), t_{k j}\left(h_{j}\right) v_{j}\right)$. Thus

$$
\begin{aligned}
D N_{k j}\left(Y_{j}^{\ell, h}+Y_{j}^{\ell, v}\right)= & D \phi_{k j}\left(h_{j}\right) Y_{j}^{\ell, h}\left(h_{j}, v_{j}\right)+t_{k j}\left(h_{j}\right) Y_{j}^{\ell, v}\left(h_{j}, v_{j}\right) \\
& +\sum_{j=1}^{n} \sum_{r, s=1}^{d} \frac{\partial t_{k j, r s}\left(h_{j}\right)}{\partial h_{j, m}} Y_{j, m}^{\ell, h}\left(h_{j}, v_{j}\right) v_{j, s} \frac{\partial}{\partial v_{k, r}},
\end{aligned}
$$

where the last term is in $C^{0}\left(U_{k} \cap U_{j}, N_{C} \otimes S^{\ell+1} N_{C}^{*}\right)$. When $N_{C}$ is flat, we see that $D N_{k j} Y_{j}=$ $Y_{k}$ if and only if $D N_{k j} Y_{j}^{\ell}=Y_{k}^{\ell}$ for each $\ell$ and that the latter holds if and only if

$$
Y_{k}^{\ell, h}\left(\phi_{k j}\left(h_{j}\right), t_{k j} v_{j}\right)=D \phi_{k j}\left(h_{j}\right) Y_{j}^{\ell, h}\left(h_{j}, v_{j}\right), \quad Y_{k}^{\ell, v}\left(\phi_{k j}\left(h_{j}\right), t_{k j} v_{j}\right)=t_{k j} Y_{j}^{\ell, v}\left(h_{j}, v_{j}\right) .
$$

In other words, $\left\{Y_{j}^{\ell}\right\}_{j}$ defines a global section of $T_{C} M \otimes S^{\ell} N_{C}^{*}$.

Lemma 2.13. Let $F_{j}$ be a formal automorphism of $\tilde{V}_{j}$ in $N_{C}$, which is tangent to identity and preserves the splitting of $T_{C}\left(N_{C}\right)$ along $U_{j}$. Then, $F_{j}$ is the time-1 map of a unique formal vector field $Y_{j}$ in $\tilde{V}_{j}$, vanishing on $U_{j}$ up to order $\geq 2$.

Proof. Let $F_{j}$ be given by

$$
\tilde{h}_{j}=h_{j}+\sum_{|\alpha| \geq 2} A_{j, \alpha}\left(h_{j}\right) v_{j}^{\alpha}, \quad \tilde{v}_{j}=v_{j}+\sum_{|\beta| \geq 2} B_{j, \beta}\left(h_{j}\right) v_{j}^{\beta} .
$$

Drop the index $j$. We want to express it as the time- 1 map of a tangent vector field

$$
Y=\sum_{\ell \geq 2}\left\{\sum_{m=1}^{n} Y_{m}^{\ell, h}(h, v) \frac{\partial}{\partial h_{m}}+\sum_{r=1}^{d} Y_{r}^{\ell, v}(h, v) \frac{\partial}{\partial v_{r}}\right\},
$$

where $Y_{m}^{\ell, h}(h, v), Y_{r}^{\ell, v}(h, v)$ are homogeneous polynomials in $v$ of degree $\ell$. The flow of $Y$ with time $\theta$ is given by

$$
h_{m}^{\theta}=h_{m}+\sum_{|\alpha| \geq 2} A_{m, \alpha}^{\theta}(h) v^{\alpha}, \quad v_{r}^{\theta}=v_{r}+\sum_{|\alpha| \geq 2} B_{r, \alpha}^{\theta}(h) v^{\alpha},
$$


where $A^{\theta}, B^{\theta}$ satisfy $A^{0}=B^{0}=0$ and

$$
\sum_{|\alpha| \geq 2} v_{j}^{\alpha} \frac{d A_{m, \alpha}^{\theta}\left(h_{j}\right)}{d \theta}=\sum_{\ell \geq 2} Y_{m}^{\ell, h}\left(h^{\theta}, v^{\theta}\right), \quad \sum_{|\alpha| \geq 2} v_{j}^{\alpha} \frac{d B_{r, \alpha}^{\theta}(h)}{d \theta}=Y_{r}^{\ell, v}\left(h^{\theta}, v^{\theta}\right) .
$$

Inductively, we can verify that $A_{m, \alpha}^{1}-Y_{m, \alpha}^{h}, B_{m, \alpha}^{1}-Y_{r, \alpha}^{v}$ are uniquely determined by $Y_{m^{\prime}, \beta}^{\ell, h}, Y_{r^{\prime}, \beta}^{\ell, v}$ with $\ell<|\alpha|$.

Note that the formal time-1 mapping of $D N_{k j}\left(Y_{j}\right)$ on $\tilde{V}_{k} \cap \tilde{V}_{j}$ can also be defined and it equals $N_{k j} F_{j} N_{k j}^{-1}$ where $F_{j}$ is the time-1 map of $Y_{j}$. Thus the uniqueness assertion in the lemma implies the following.

Proposition 2.14. Any automorphism $F$ of a formal neighborhood of $C$ in $N_{C}$, which is tangent to identity and preserves the splitting of $T_{C}\left(N_{C}\right)$, is the time-1 map of a unique vector field defined on a formal neighborhood of $C$ in $N_{C}$ and vanishing on $C$. Assume further that $N_{C}$ is flat. Then any tangent vector field $Y$ of $N_{C}$ that vanishes on $C$ to order two admits a decomposition

$$
Y=\sum_{\ell \geq 2} Y^{\ell}, \quad Y^{\ell} \in H^{0}\left(C, T_{C} M \otimes S^{\ell} N_{C}^{*}\right) .
$$

We write $\delta_{m}=\left(\delta_{m}^{h}, \delta_{m}^{v}\right)$ corresponding to the splitting $T_{C} M=T C \oplus N_{C}$. Let us set $\mathcal{G}_{m}:=$ Range $\left(\delta_{m}\right)$. We have a decomposition

$$
Z^{1}\left(\mathcal{U}, T_{C} M \otimes S^{m} N_{C}^{*}\right)=\mathcal{G}_{m} \oplus \mathcal{N}_{m}
$$

where $\mathcal{N}_{m} \simeq H^{1}\left(\mathcal{U}, T M_{C} \otimes S^{m} N_{C}^{*}\right)$. Let $C^{0}\left(\mathcal{U}, T M_{C} \otimes S^{m} N_{C}^{*}\right)=\mathcal{R}_{m} \oplus \operatorname{ker} \delta_{m}$ with $\delta_{m}\left(\mathcal{R}_{m}\right)=\mathcal{G}_{m}$. We emphasize that the decomposition (2.29) is not unique. For our convergence result, a natural decomposition will be given via a possibly non-unique minimizing solution. Consequently, $\oplus$ is interpreted as merely a decomposition suitable for convergence proof.

Lemma 2.15. Suppose that $N_{C}$ is flat. Any formal transformation $F_{j}$ of $\tilde{V}_{j}$ which is tangent to identity and preserves the splitting of $T_{C}\left(N_{C}\right)$ can be uniquely factorized as

$$
F_{j}=G_{j}^{-1} \circ H_{j}
$$

where $H_{j}-I \in \sum_{m \geq 2} \mathcal{R}^{m}, G_{j}$ is an automorphism of $\tilde{V}_{j}$, and terms of order $m$ in $G_{j}, H_{j}$ are uniquely determined by the terms of order at most $m$ in $F_{j}$. Furthermore, $G_{i} N_{i k}=N_{i k} G_{k}$ for all $i, k$.

Proof. We know that $F_{j}=\exp \sum_{m} C_{j}^{m}$ is the time-1 map of $\sum_{m \geq 2} C_{j}^{m}$.

We want to decompose

$$
\exp \sum_{m} C_{j}^{m}=\left(\exp \sum_{m} A_{j}^{m}\right)\left(I+\sum_{m} H_{j}^{m}\right) .
$$

By Campbell-Hausdorff formula, we are led to the equation

$$
H_{j}^{m}=C_{j}^{m}-A_{j}^{m}+E_{j}^{m}
$$

where $E_{j}^{m}$ depends only on $C_{j}^{\ell}, A_{j}^{\ell}$ for $\ell<m$. We determine $A_{j}^{m}, B_{j}^{m}$ by decomposing $C_{j}^{m}$ and $E_{j}^{m}$ as follow : Let $\pi$ be the (non-canonical) projection from $C^{0}\left(\mathcal{U}, T M_{C} \otimes S^{m} N_{C}^{*}\right)$ onto ker $\delta_{m}$. Let $\left\{A_{j}^{m}\right\}_{j}:=\pi\left(\left\{C_{j}^{m}+E_{j}^{m}\right\}\right)$. Then $\left\{H_{j}^{m}\right\} \in \mathcal{R}_{m}$. 
Next, we study the dependence of cohomology classes of $\left[\phi_{k j}^{h}\right]^{\ell},\left[\phi_{k j}^{v}\right]^{\ell}$ in coordinates. We first consider the full set of linear cohomological equations.

2.9. Formal coordinates in the absence of formal obstructions. For a power series $u\left(z_{j}, w_{j}\right)$, let $u^{\leq m}\left(z_{j}, w_{j}\right)$ be the Taylor polynomial of $u$ about $w_{j}=0$ with degree $m$. Thus we can define

$$
u=u^{\leq m}+u^{>m}, \quad u^{>m}\left(z_{j}, w_{j}\right)=O\left(\left|w_{j}\right|^{m+1}\right), \quad[u]^{m}=u^{\leq m}-u^{<m}, \quad[u]_{\ell}^{m}=u^{\leq m}-u^{<\ell} .
$$

In order to describe the coboundary operator in next lemma, we define the linear operator $\widetilde{D}$ by

$$
((\widetilde{D} u) f)\left(h_{j}, v_{j}\right):=\frac{\partial u}{\partial h_{j}}\left(h_{j}, 0\right) f^{h}\left(h_{j}, v_{j}\right)+\frac{\partial u}{\partial v_{j}}\left(h_{j}, 0\right) f^{v}\left(h_{j}, v_{j}\right),
$$

for a function $u\left(h_{j}, v_{j}\right)$. The standard differential $D$ is given by

$$
((D u) f)\left(h_{j}, v_{j}\right)=\frac{\partial u}{\partial h_{j}}\left(h_{j}, v_{j}\right) f^{h}\left(h_{j}, v_{j}\right)+\frac{\partial u}{\partial v_{j}}\left(h_{j}, v_{j}\right) f^{v}\left(h_{j}, v_{j}\right) .
$$

Thus

$$
(D u-\widetilde{D} u) f\left(h_{j}, v_{j}\right)=\left(D u\left(h_{j}, v_{j}\right)-D u\left(h_{j}, 0\right)\right) f\left(h_{j}, v_{j}\right) .
$$

For a multiindex $\alpha=\left(\alpha_{h}, \alpha_{v}\right)$, define

$$
\left(\tilde{D}^{\alpha} u\right)\left(h_{j}\right)=\left\{\frac{\partial^{|\alpha|} u}{\partial h_{j}^{\alpha_{h}} \partial v_{j}^{\alpha_{v}}}\right\}\left(h_{j}, 0\right) .
$$

Lemma 2.16. Let $\Phi_{k j}=N_{k j}+\phi_{k j}$ satisfy condition $L_{m}$ with $m \geq 1$. Suppose that $F_{j}\left(h_{j}, v_{j}\right)=\left(h_{j}, v_{j}\right)+f_{j}\left(h_{j}, v_{j}\right)$ with $f_{j}\left(h_{j}, v_{j}\right)=O\left(\left|v_{j}\right|^{2}\right)$ are formal mappings such that $\left\{F_{k}^{-1} \Phi_{k j} F_{j}\right\} \in L_{m}$. Then, on $U_{j} \cap U_{k}, l=2, \ldots, m$,

$$
\begin{aligned}
\left(\delta \left\{\left[f_{j}\right]\right.\right. & \leq l\})_{k j}\left(h_{j}, v_{j}\right)=-\left[N_{k j}\left(\left(I+\left[f_{j}\right] \leq l-2\right)\left(h_{j}, v_{j}\right)\right)-N_{k j}\left(h_{j}, v_{j}\right)\right. \\
& \left.-D N_{k j}\left(h_{j}, v_{j}\right)\left[f_{j}\right]^{\leq l-2}\left(h_{j}, v_{j}\right)\right]^{\leq l}-\left(0,\left(D t_{k j}\left(h_{j}\right)\left[f_{j}^{h}\right]^{\leq l-1}\left(h_{j}, v_{j}\right)\right) v_{j}\right) .
\end{aligned}
$$

(a) If $f_{j}\left(h_{j}, v_{j}\right)=O\left(\left|v_{j}\right|^{m+1}\right)$ for all $j$, then $N_{k j}+\tilde{\phi}_{k j}=F_{k}^{-1} \Phi_{k j} F_{j}+O\left(\left|v_{j}\right|^{2 m+1}\right)$ hold if and only if on $U_{j} \cap U_{k}$

$$
\left(\delta\left\{\left[f_{i}\right]^{\leq 2 m}\right\}\right)_{k j}=\left[\tilde{\phi}_{k j}-\phi_{k j}\right]^{\leq 2 m}-\left(0,\left(D t_{k j}\left(h_{j}\right)\left[f_{j}^{h}\right]^{\leq 2 m-1}\right) v_{j}\right) .
$$

(b) If $\left\{F_{j}\right\}$ defines a germ of biholomorphism of order $m$ at the zero section of the normal bundle, i.e.

$$
F_{k}^{-1} N_{k j} F_{j}\left(h_{j}, v_{j}\right)=N_{k j}\left(h_{j}, v_{j}\right)+O\left(\left|v_{j}\right|^{m+1}\right)
$$

and if $f_{j}^{h}\left(h_{j}, v_{j}\right)=O\left(\left|v_{j}\right|^{m}\right)$, then $\mathcal{V}_{j}^{\leq m}\left(h_{j}, v_{j}\right):=\left(h_{j}, v_{j}+\left[f_{j}^{v}\right]^{\leq m}\right)$ preserves $\left\{N_{k j}\right\}$.

(c) Suppose $F_{k}^{-1} \Phi_{k j} F_{j} \in L_{2 m}$. Assume further that either $N_{C}$ is flat or

$$
H^{0}\left(C, T C \otimes S^{p} N_{C}^{*}\right)=0, \quad 2 \leq p \leq 2 m .
$$


Then there exist $\hat{F}_{j}=I+O\left(\left|v_{j}\right|^{m+1}\right)$ where $\left[\hat{F}_{j}^{h}\right]_{m+1}^{2 m}$ are uniquely determined by $\left[\Phi_{k j}\right]_{m+1}^{2 m}$ such that $\hat{F}_{k}^{-1} \Phi_{k j} \hat{F}_{j} \in L_{2 m}$. There exists a unique decomposition $\left\{\hat{F}_{j}=\right.$ $\left.\mathcal{H}_{j} \circ \mathcal{V}_{j} \circ \tilde{F}_{j}\right\}$ in the form

$$
\begin{gathered}
\mathcal{H}_{j}\left(h_{j}, v_{j}\right)=\left(h_{j}+H_{j}\left(h_{j}, v_{j}\right), v_{j}\right), \\
\mathcal{V}_{j}\left(h_{j}, v_{j}\right)=\left(h_{j}, v_{j}+V_{j}\left(h_{j}, v_{j}\right)\right), \\
{\left[\tilde{F}_{j}\right]^{i}=0, \quad \forall 2 \leq i \leq 2 m, \quad\left[H_{j}\right]^{\ell}=\left[V_{j}\right]^{\ell}=0, \quad \forall \ell>2 m .}
\end{gathered}
$$

Furthermore, $\left[H_{j}\right]^{\ell}=\left[V_{j}\right]^{\ell}=0$ for $\ell \leq m$, and $H_{j}$ are uniquely determined by

$$
\left(\delta^{h}\left\{H_{i}\right\}\right)_{k j}=-\left[\phi_{k j}^{h}\right]^{\leq 2 m} .
$$

Moreover, $\tilde{\phi}_{k j}=\mathcal{H}_{k}^{-1} \Phi_{k j} \mathcal{H}_{j}-N_{k j}$ satisfy $\tilde{\phi}_{k j}^{h}\left(h_{j}, v_{j}\right)=O\left(\left|v_{j}\right|^{2 m+1}\right)$ and $\tilde{\phi}_{k j}^{v}\left(h_{j}, v_{j}\right)=$ $O\left(\left|v_{j}\right|^{m+1}\right)$, and $V_{i}$ satisfy

$$
\left(\delta^{v}\left\{V_{i}\right\}\right)_{k j}=-\left[\tilde{\phi}_{k j}^{v}\right]^{\leq 2 m} .
$$

Proof. Let $\Phi_{k j}=N_{k j}+\phi_{k j}$ and $\tilde{\Phi}_{k j}=N_{k j}+\tilde{\phi}_{k j}$. Suppose that both $\phi_{k j}$ and $\tilde{\phi}_{k j}$ are of order $\geq m+1$ (i.e. $\left.O\left(\left|v_{j}\right|^{m+1}\right)\right)$ and $F_{k} \Phi_{k j}=\tilde{\Phi}_{k j} F_{j}$. Recall that $F_{k}=I+f_{k}$. To use the coboundary operator, we write

$$
\begin{aligned}
f_{k}\left(N_{k j}\right)-\widetilde{D} N_{k j} f_{j}+\phi_{k j}-\tilde{\phi}_{k j}= & \underbrace{\left(f_{k}\left(N_{k j}-f_{k}\left(N_{k j}+\phi_{k j}\right)\right)\right)}_{A} \\
& +\underbrace{\left(\tilde{\phi}_{k j}\left(I+f_{j}\right)-\tilde{\phi}_{k j}\right)}_{B} \\
& +\underbrace{\left(N_{k j}\left(I+f_{j}\right)-N_{k j}-\tilde{D} N_{k j} f_{j}\right)}_{C} .
\end{aligned}
$$

Since $f_{j}$ has order $\geq 2$ at $v_{j}=0$, by the Taylor expansion at $N_{k j}$ and at $I$ respectively, both $A$ and $B$ are of order $\geq m+2$ (w.r.t $v_{j}$ ) at the origin. For the same reason, the $C$ is of order $\geq 4$. We recall that, for each $\ell \in \mathbb{N}^{*}$, the coboundary operator $\delta$ sends $C^{0}\left(\mathcal{U}, T_{C} M \otimes S^{\ell}\left(N_{C}^{*}\right)\right)$ into $C^{1}\left(\mathcal{U}, T_{C} M \otimes S^{\ell}\left(N_{C}^{*}\right)\right)$ as sections. It is defined in coordinates by

$$
(\delta f)_{k j}=\widetilde{D} N_{k j} f_{j}\left(h_{j}, v_{j}\right)-f_{k}\left(N_{k j}\left(h_{j}, v_{j}\right)\right)
$$

on $U_{j} \cap U_{k}$ when $f=\left\{f_{j}\right\} \in C^{0}\left(\mathcal{U}, T_{C} M \otimes S^{\ell}\left(N_{C}^{*}\right)\right.$ ). As $\delta$ preserves the degree $\ell$ of $f_{j}$ in $v_{j}$, we shall omit its dependence in $\ell$. Truncating the Taylor expansion of (2.39) at $v_{j}=0$ up to degree $m$ will lead to the first point.

Since $f_{j}\left(h_{j}, v_{j}\right)=O\left(\left|v_{j}\right|^{2}\right)$, then $A, B$ are of order $\geq m+1$. Using (2.30), we obtain

$$
\begin{aligned}
C= & N_{k j}\left(I+f_{j}\left(h_{j}, v_{j}\right)\right)-N_{k j}\left(h_{j}, v_{j}\right)-D N_{k j}\left(h_{j}, v_{j}\right) f_{j}\left(h_{j}, v_{j}\right) \\
& +\left(D N_{k j}\left(h_{j}, v_{j}\right)-D N_{k j}\left(h_{j}, 0\right)\right) f_{j}\left(h_{j}, v_{j}\right) .
\end{aligned}
$$

We have $\left(D N_{k j}\left(h_{j}, v_{j}\right)-D N_{k j}\left(h_{j}, 0\right)\right) f_{j}\left(h_{j}, v_{j}\right)=\left(0, D t_{k j}\left(h_{j}\right) f_{j}^{h}\left(h_{j}, v_{j}\right) v_{j}\right)$. Thus,

$$
C=\left(0,\left(D t_{k j}\left(h_{j}\right) f_{j}^{h}\left(h_{j}, v_{j}\right) v_{j}\right)+a(1)-a(0)-a^{\prime}(0)\right.
$$


with $a(\lambda)=N_{k j}\left(h_{j}+\lambda f_{j}^{h}, v_{j}+\lambda f_{j}^{v}\right)$. Note that

$$
\begin{aligned}
a(1)-a & (0)-a^{\prime}(0)=\int_{0}^{1}(1-\lambda) a^{\prime \prime}(\lambda) d \lambda \\
= & \sum_{|\alpha|=2} \frac{|\alpha| !}{\alpha !} \int_{0}^{1}(1-\lambda) D^{\alpha} N_{k j}\left(I+\lambda f_{j}\right) f_{j}^{\alpha} d \lambda \\
= & \sum_{|\alpha|=2} \frac{|\alpha| !}{\alpha !} \int_{0}^{1}(1-\lambda) D^{\alpha} N_{k j}\left(I+\lambda\left[f_{j}\right]^{\leq m-2}\right)\left(\left[f_{j}\right]^{\leq m-2}\right)^{\alpha} d \lambda+O\left(\left|v_{j}\right|^{m+1}\right) \\
= & b(1)-b(0)-b^{\prime}(0)+O\left(\left|v_{j}\right|^{m+1}\right)
\end{aligned}
$$

for $b(\lambda)=N_{k j}\left(I+\lambda\left[f_{j}\right]^{\leq m-2}\right)$. This proves $(2.31)$.

For point (a), we use (2.39) again. This time, we have $A\left(h_{j}, v_{j}\right)=O\left(\left|v_{j}\right|^{2 m+1}\right)$ and $B\left(h_{j}, v_{j}\right)=O\left(\left|v_{j}\right|^{2 m+1}\right)$, while $C=\left(0, D N_{k j}\left(h_{j}\right)\left[f_{j}^{h}\right]^{\leq 2 m-1} v_{j}\right)+O\left(\left|v_{j}\right|^{2 m+1}\right)$. We have derived (2.32).

For point (b), note that $F_{k}^{-1} N_{k j} F_{j}=N_{k j}+O\left(\left|v_{j}\right|^{m+1}\right)$ is equivalent to $F_{k} N_{k j}=N_{k j} F_{j}+$ $O\left(\left|v_{j}\right|^{m+1}\right)$. From the vertical components, we obtain

$$
t_{k j}\left(h_{j}\right) v_{j}+f_{k}^{v}\left(\varphi_{k j}\left(h_{j}\right), t_{k j}\left(h_{j}\right) v_{j}\right)=t_{k j}\left(h_{j}+f_{j}^{h}\right)\left(v_{j}+f_{j}^{v}\left(h_{j}, v_{j}\right)\right)+O\left(\left|v_{j}\right|^{m+1}\right) .
$$

Since $f_{j}^{h}=O\left(\left|v_{j}\right|^{m}\right)$ and $f_{j}^{v}=O\left(\left|v_{j}\right|^{2}\right)$, the $m$-jet (w.r.t. $\left.v_{j}\right)$ above reads

$$
t_{k j}\left(h_{j}\right) v_{j}+\left[f_{k}^{v}\right]^{\leq m}\left(\varphi_{k j}\left(h_{j}\right), t_{k j}\left(h_{j}\right) v_{j}\right)=t_{k j}\left(h_{j}\right)\left(v_{j}+\left[f_{j}^{v}\right]^{\leq m}\left(h_{j}, v_{j}\right)\right) .
$$

That is that $\mathcal{V}_{k}^{\leq m} N_{k j}=N_{k j} \mathcal{V}_{j}^{\leq m}$, as $\mathcal{V}_{j}^{\leq m}\left(h_{j}, v_{j}\right)=\left(h_{j}, v_{j}+\left[f_{j}\right]^{\leq m}\left(h_{j}, v_{j}\right)\right)$.

The point (c) follows from Proposition 2.14 when $N_{C}$ is flat. For the remaining case, it follows from points (a) and (b) as follows.

By $(2.32)$ and $H^{0}\left(C, T C \otimes S^{\ell} N_{C}^{*}\right)=0$, we obtain $\left[f_{j}^{h}\right]_{2}^{m}=0$. By $(b)$, we know that $\left[F_{j}\right] \leq m$ preserve $N_{k j}$. Then $\hat{F}_{j}=F_{j}\left(\left[F_{j}\right]^{\leq m}\right)^{-1}$ meet the requirement. The uniqueness of $\left[\hat{F}_{j}^{h}\right]^{\ell}$ for $m<\ell \leq 2 m$ follows from the assumption on $H^{0}$ too.

We are seeking a unique decomposition $F_{j}=\mathcal{H}_{j} \circ \mathcal{V}_{j} \circ \tilde{F}_{j}$. Let us write $F_{k}^{-1} \Phi_{k j} F_{j}=$ $N_{k j}+\tilde{\phi}_{k j}$ with $\tilde{\phi}_{k j}=O\left(\left|v_{j}\right|^{2 m+1}\right)$. From the horizontal component of (2.32) in which $\left[\tilde{\phi}_{k j}^{h}\right] \leq 2 m=0$ and condition (2.33), we uniquely determine $\left\{\left[f_{j}^{h}\right]^{\leq 2 m}\right\}$. Take $\mathcal{H}_{j}\left(h_{j}, v_{j}\right)=$ $\left(h_{j}+\left[f_{j}\right] \leq 2 m\left(h_{j}, v_{j}\right), v_{j}\right)$. Then

$$
\mathcal{H}_{k}^{-1} \Phi_{k j} \mathcal{H}_{j}\left(h_{j}, v_{j}\right)=\left(\varphi_{k j}\left(h_{j}\right), t_{k j}\left(h_{j}\right) v_{j}+\tilde{\phi}_{k j}^{v}\left(h_{j}, v_{j}\right)\right)+O\left(\left|v_{j}\right|^{2 m+1}\right) .
$$

We still have $\left(\mathcal{H}_{k}^{-1} F_{k}\right)^{-1}\left(\mathcal{H}_{k}^{-1} \Phi_{k j} \mathcal{H}_{j}\right)\left(\mathcal{H}_{j}^{-1} F_{j}\right) \in L_{2 m}$. We have

$$
\mathcal{H}_{j}^{-1} F_{j}\left(h_{j}, v_{j}\right)=\mathcal{V}_{j}\left(h_{j}, v_{j}\right)+O\left(\left|v_{j}\right|^{2 m+1}\right), \quad \mathcal{V}_{j}\left(h_{j}, v_{j}\right)=\left(h_{j}, v_{j}+V_{j}\left(h_{j}, v_{j}\right)\right),
$$

where $\tilde{\phi}_{k j}^{v}, V_{j}$ contain only terms of orders $\ell$ in $v_{j}$ for $m+1 \leq \ell \leq 2 m$.

Since $F_{j}=\mathcal{H}_{j} \mathcal{V}_{j}+O\left(\left|v_{j}\right|^{2 m+1}\right)$, we have

$$
\mathcal{V}_{k}^{-1}\left(\mathcal{H}_{k}^{-1} \Phi_{k j} \mathcal{H}_{j}\right) \mathcal{V}_{j} \in L_{2 m}
$$

From the vertical components of (2.40)-(2.41), and (2.32) in which we take $D t_{k j}\left[f_{j}^{h}\right] \leq 2 m-1=$ 0 , we see that $(2.32)$ becomes $(2.38)$, i.e. $\left(\delta^{v}[V]^{\ell}\right)_{k j}=-\left[\tilde{\phi}_{k j}^{v}\right]^{\ell}$ for $\ell=m+1, \ldots, 2 m$. To 
show the uniqueness of $\left[F_{j}\right] \leq 2 m$, we may assume that $\Phi_{k j}=N_{k j}+O\left(\left|v_{j}\right|^{2 m+1}\right)$. Then the uniqueness follows from the above arguments.

The following is in Ueda [40], when both the dimension and codimension of $C$ are one.

Lemma 2.17. Let $\Phi_{k j}$ satisfy condition $V_{m}$ with $m \geq 1$. Suppose that $N_{C}$ is flat and $H^{0}\left(C, N_{C} \otimes S^{\ell}\left(N_{C}^{*}\right)\right)=0$ for $1<\ell \leq m$. Then $\left[\phi_{k j}^{v}\right]^{m+1} \in H^{1}\left(\mathcal{U}, N_{C} \otimes S^{m+1}\left(N_{C}^{*}\right)\right)$ is independent of coordinates of the neighborhoods of $C$. Furthermore, there are formal biholomorphic mappings $F_{j}=I+\left(f_{j}^{h}, f_{j}^{v}\right)$ with $f_{j}\left(h_{j}, v_{j}\right)=O\left(\left|v_{j}\right|^{2}\right)$ satisfy

$$
\left\{F_{k}^{-1} \Phi_{k j} F_{j}\right\} \in V_{m+1}
$$

if and only if $\left[\phi_{k j}^{v}\right]^{m+1}=0$ in $H^{1}\left(\mathcal{U}, N_{C} \otimes S^{m+1}\left(N_{C}^{*}\right)\right)$. When (2.42) holds, $\left\{\tilde{F}_{k}^{-1} \Phi_{k j} \tilde{F}_{j}\right\}$ is still in $V_{m+1}$, for

$$
\tilde{F}_{j}\left(h_{j}, v_{j}\right)=\left(h_{j}, v_{j}+\left[f_{j}^{v}\right]^{m+1}\left(h_{j}, v_{j}\right)\right) .
$$

Proof. Let $\tilde{\Phi}_{k j}:=F_{k}^{-1} \Phi_{k j} F_{j}$. We want to show that

$$
\left[\tilde{\phi}_{k j}^{v}\right]^{m+1}=\left[\phi_{k j}^{v}\right]^{m+1} \quad \text { in } H^{1}\left(\mathcal{U}, N_{C} \otimes S^{m+1}\left(N_{C}^{*}\right)\right),
$$

provided that $\tilde{\Phi}_{k j}\left(h_{j}, v_{j}\right)=N_{k j}\left(h_{j}, v_{j}\right)+\left(\tilde{\phi}_{k j}^{h}, \tilde{\phi}_{k j}^{v}\right), \Phi_{k j}\left(h_{j}, v_{j}\right)=N_{k j}\left(h_{j}, v_{j}\right)+\left(\phi_{k j}^{h}, \phi_{k j}^{v}\right)$, and

$$
\tilde{\phi}_{k j}^{v}\left(h_{j}, v_{j}\right)=O\left(\left|v_{j}\right|^{m+1}\right), \quad \phi_{k j}^{v}\left(h_{j}, v_{j}\right)=O\left(\left|v_{j}\right|^{m+1}\right) .
$$

First, we have $F_{j}\left(h_{j}, v_{j}\right)=\left(h_{j}, v_{j}\right)+O\left(\left|v_{j}\right|^{2}\right)$. Suppose that $\left[f_{j}^{v}\right]^{\leq m_{*}-1}=0$ for $2 \leq m_{*} \leq$ $m$. Comparing vertical components of $\Phi_{k j} \circ F_{j}=F_{k} \circ \tilde{\Phi}_{k j}$, we obtain

$$
\begin{aligned}
& {\left[t_{k j} \cdot\left(v_{j}+f_{j}^{v}\left(h_{j}, v_{j}\right)\right)\right]^{\leq m_{*}}=\left(\Phi_{k j}^{v} \circ F_{j}\right) \leq m_{*}\left(h_{j}, v_{j}\right)} \\
& \quad=\left(F_{k}^{v} \circ \tilde{\Phi}_{k j}\right)^{\leq m_{*}}\left(h_{j}, v_{j}\right)=\left(F_{k}^{v}\right)^{\leq m_{*}} \circ N_{k j}\left(h_{j}, v_{j}\right) .
\end{aligned}
$$

Here the last identity is obtained from $\tilde{\Phi}_{k j}\left(h_{j}, v_{j}\right)-N_{k j}\left(h_{j}, v_{j}\right)=O\left(\left|v_{j}\right|^{2}\right),\left[F_{j}^{v}\right] \leq m_{*}\left(h_{j}, v_{j}\right)=$ $v_{j}+\left[f_{j}^{v}\right]^{m_{*}}$, and (2.43). Looking at terms of order $m_{*}$ in $w_{j}$, we see that $\left\{\left[f_{j}^{v}\right]^{\ell}\right\}$ is a global section of $N_{C} \otimes S^{\ell}\left(N_{C}^{*}\right)$ for $\ell=m_{*}$. This shows that $\left[f_{j}^{v}\right] \leq m_{*}=0$ and we can take $m_{*}=m$, i.e. $\left[f_{j}^{v}\right]^{\leq m}=0$.

We also have $\left[\Phi_{k j}^{v} F_{j}\right]^{m+1}=t_{k j}\left[f_{j}^{v}\right]^{m+1}+\left[\phi_{k j}^{v}\right]^{m+1}$ and $\left[F_{k}^{v} \tilde{\Phi}_{k j}\right]^{m+1}=\left[f_{k}^{v}\right]^{m+1} \circ N_{k j}+$ $\left[\tilde{\phi}_{k j}^{v}\right]^{m+1}$. This shows that

$$
\left[\tilde{\phi}_{k j}^{v}\right]^{m+1}-\left[\phi_{k j}^{v}\right]^{m+1}=t_{k j}\left[f_{j}^{v}\right]^{m+1}-\left[f_{k}^{v}\right]^{m+1} \circ N_{k j} .
$$

The latter is equivalent to $\left[\tilde{\phi}_{k j}^{v}\right]^{m+1}=\left[\phi_{k j}^{v}\right]^{m+1}$ in $H^{1}\left(\mathcal{U}, N_{C} \otimes S^{m+1}\left(N_{C}^{*}\right)\right)$, which follows from Lemma $2.7(b)$. The last assertion is equivalent to $(2.44)$ with $\left[\tilde{\phi}_{k j}^{v}\right]^{m+1}=0$.

\section{A MAJORANT METHOD FOR THE VERTICAL LINEARIZATION}

Let $C$ be an $n$-dimensional complex compact manifold embedded in an $(n+d)$-dimensional complex manifold. We assume that the normal bundle $N_{C}$ is flat and unitary. Let $\left\{t_{k j}\right\}$ be its transition (constant) matrices in a suitable covering $\mathcal{U}=\left\{U_{j}\right\}$ of $C$, we have 
$t_{k j} t_{k j}^{*}=\mathrm{Id}$. Let $K\left(N_{C} \otimes S^{m}\left(N_{C}^{*}\right)\right)$ be the "norm" of the cohomological operator acting on $C^{0}\left(\mathcal{U}, N_{C} \otimes S^{m}\left(N_{C}^{*}\right)\right)$ as defined in Theorem A.12. Let us consider the sequence of numbers $\left\{\eta_{m}\right\}_{m \geq 1}$ with $\eta_{1}=1$ and

$$
\eta_{m}=K\left(N_{C} \otimes S^{m}\left(N_{C}^{*}\right)\right)_{m_{1}+\cdots+m_{p}+s=m} \eta_{m_{1}} \cdots \eta_{m_{p}}, \quad m>1,
$$

where $1 \leq m_{i}<m$ for all $i$ and $s \in \mathbb{N}$.

In this section, we shall prove the following

Theorem 3.1. Let $C$ be a compact complex submanifold in $M$ with $T_{C} M=T C \oplus N_{C}$. Assume that the embedding is vertically linearizable by a formal holomorphic mapping which is tangent to the identity and preserves the splitting of $T_{C} M$ or that $H^{1}\left(C, N_{C} \otimes S^{\ell}\left(N_{C}^{*}\right)\right)=0$ for all $\ell \geq 2$. We also assume that $N_{C}$ is unitary flat and that $H^{0}\left(C, N_{C} \otimes S^{\ell}\left(N_{C}^{*}\right)\right)=0$ for all $\ell \geq 2$. Assume that for the $\eta_{m}$ defined above, there are positive constants $L_{0}, L$ such that $\eta_{m} \leq L_{0} L^{m}$ for all $m$. Then the embedding is actually holomorphically vertically linearizable.

Remark 3.2. In the previous Theorem 3.1, if a neighborhood of $C$ is formally vertically linearizable by a minimizing vertical mapping which is tangent to the identity and preserves the splitting of $T_{C} M$, then the assumption " $H^{0}\left(C, N_{C} \otimes S^{\ell}\left(N_{C}^{*}\right)\right)=0, \ell>1$ " is not necessary. Here by a formal minimizing vertical mapping it means a map of the form $\left(h_{j}, v_{j}+f_{j}^{v}\left(h_{j}, v_{j}\right)\right)$ with $\left\{f_{j}^{v}\right\} \in C^{0}\left(C, \bigoplus_{\ell \geq 2} N_{C} \otimes S^{\ell}\left(N_{C}^{*}\right)\right)$ such that each $\left\{\left[f_{j}^{v}\right]^{\ell}\right\}_{j}$ is a possibly non-unique Donin (minimizing) solution of a suitable cohomology equation.

Corollary 3.3. Under assumptions of Theorem 3.1, there exists, in a neighborhood of $C$ in $M$, a smooth holomorphic d-dimensional foliation having $C$ as a leaf.

Proof. According to Theorem 3.1, there is a neighborhood of the $C$ in $M$ with suitable holomorphic coordinates patches $\left(V_{j},\left(h_{j}, v_{j}\right)\right)$ with $\left(h_{j}, v_{j}\right) \in \mathbb{C}^{n} \times \mathbb{C}^{d}$ and $C \cap V_{j}=\left\{v_{j}=0\right\}$, such that, on $V_{j} \cap V_{k}$, we have

$$
v_{k}=t_{k j} v_{j}, \quad h_{k}=\tilde{\varphi}_{k j}\left(h_{j}, v_{j}\right) .
$$

We then define the foliation in chart $V_{j}$ by $d v_{j}=0$.

The rest of the section is devoted to the proof of Theorem 3.1. We follow the method of majorant developed by T. Ueda [40] for 1-dimensional unitary normal bundle over compact complex curve.

3.1. Conjugacy equations and cohomological equations. Let us first recall (2.21) and $(2.20)$ :

$$
\mathcal{L}_{k j}^{v}\left(f_{j}^{v}\right)=\phi_{k j}^{v}\left(h_{j}, v_{j}+f_{j}^{v}\right)-\left(f_{k}^{v}\left(\hat{\Phi}_{k j}^{h}\left(h_{j}, v_{j}\right), t_{k j} v_{j}\right)-f_{k}^{v}\left(\varphi_{k j}\left(h_{j}\right), t_{k j} v_{j}\right)\right)
$$

where

$$
\begin{aligned}
\hat{\Phi}_{k j}^{h}\left(h_{j}, v_{j}\right) & =\varphi_{k j}\left(h_{j}\right)+\phi_{k j}^{h}\left(h_{j}, v_{j}+f_{j}^{v}\right), \\
\mathcal{L}_{k j}^{v}\left(f_{j}^{v}\right) & =f_{k}^{v}\left(\varphi_{k j}\left(h_{j}\right), t_{k j} v_{j}\right)-t_{k j} f_{j}^{v} .
\end{aligned}
$$


Let us expand $\phi_{k j}^{h}\left(h_{j}, v_{j}+f_{j}^{v}\right)$ in power of $v_{j}$ by using

$$
\begin{aligned}
\phi_{k j}^{h}\left(h_{j}, w_{j}\right) & =: \sum_{Q \in \mathbb{N}_{2}^{d}} \phi_{k j, Q}^{h}\left(h_{j}\right) w_{j}^{Q} \\
\phi_{k j}^{h}\left(h_{j}, v_{j}+f_{j}^{v}\left(h_{j}, v_{j}\right)\right)=: & \sum_{Q \in \mathbb{N}_{2}^{d}} h_{k j, Q}^{\prime}\left(h_{j}\right) v_{j}^{Q}=: h_{k j}^{\prime}\left(h_{j}, v_{j}\right) .
\end{aligned}
$$

We have

$$
\sum_{Q \in \mathbb{N}_{2}^{d}} h_{k j, Q}^{\prime}\left(h_{j}\right) v_{j}^{Q}=\sum_{Q \in \mathbb{N}_{2}^{d}} \phi_{k j, Q}^{h}\left(h_{j}\right)\left(v_{j}+f_{j}^{v}\left(h_{j}, v_{j}\right)\right)^{Q}
$$

Let us also set

$$
\sum_{Q \in \mathbb{N}_{2}^{d}} h_{k j, Q}^{\prime \prime}\left(h_{j}\right) v_{j}^{Q}:=f_{k}^{v}\left(\hat{\Phi}_{k j}^{h}\left(h_{j}, v_{j}\right), t_{k j} v_{j}\right)-f_{k}^{v}\left(\varphi_{k j}\left(h_{j}\right), t_{k j} v_{j}\right) .
$$

As we shall see below, the functions $\left[h^{\prime}\right]^{m}$ and $\left[h^{\prime \prime}\right]^{m}$ are defined by induction on $m \geq 2$ as they depend on $[f]^{l}, l=2, \ldots, m-1$.

Therefore, the homogeneous polynomial of degree $m \geq 2$ of the Taylor expansion of solution of the conjugacy equation satisfies

$$
\mathcal{L}_{k j}^{v}\left(\left[f_{j}^{v}\right]^{m}\right)=\left[h_{k j}^{\prime}\right]^{m}+\left[h_{k j}^{\prime \prime}\right]^{m} .
$$

According to Lemma 2.17, there is a solution to the above equation either by the formal assumption or by the assumption that the cohomology class of $\left[h_{k j}^{\prime}\right]^{m}+\left[h_{k j}^{\prime \prime}\right]^{m}$ is 0 , i.e. it is a coboundary. Indeed, since the normal bundle is flat, this class is independent of the coordinates system and the neighborhood is formally vertically linearizable.

3.2. A modified Fischer norm for symmetric powers. We define a scaler product on the space of polynomials $\mathbb{C}\left[x_{1}, \ldots, x_{d}\right]$ as follows. First, we set

$$
\left\langle x^{R}, x^{Q}\right\rangle_{\mathrm{mf}}:=\left\{\begin{array}{ll}
\frac{\left(r_{1} !\right) \cdots\left(r_{d} !\right)}{|R| !} & \text { if } R=Q \\
0 & \text { otherwise }
\end{array}, \quad\left|\sum_{Q} C_{Q} x^{Q}\right|_{\mathrm{mf}}^{2}:=\sum_{Q}\left|C_{Q}\right|^{2} \frac{Q !}{|Q| !},\right.
$$

where $R=\left(r_{1}, \ldots, r_{d}\right)$ and $|R|=r_{1}+\cdots+r_{d}$, and $C_{Q}$ are constants. The subscript mf stands for "modified Fischer". The associated norm will be denoted by $|\cdot|_{k}$. The Fischer (resp. modified Fischer) scalar product has been used in [9,23,38] (resp. [28]). Let $\omega$ be an open set on $\mathbb{C}^{n}$. For a vector of polynomials $g=\left(g_{1}, \ldots, g_{k}\right) \in \mathcal{O}^{k}(\omega) \otimes \mathbb{C}\left[x_{1}, \ldots, x_{d}\right]$, we set

$$
|g|_{\mathrm{mf}, \omega}^{2}:=\sup _{z \in \omega}|g(z, \cdot)|_{\mathrm{mf}}^{2}:=\sup _{z \in \omega} \sum_{j=1}^{k} \sum_{Q \in \mathbb{N}^{d}} \frac{Q !}{|Q| !}\left|g_{j, Q}(z)\right|^{2} .
$$

We now apply the Fischer norm (resp. modified Fischer norm) to $f \in C^{q}\left(\mathcal{U}, E \otimes S^{L} N_{C}^{*}\right)$. Returning to notation in (2.25), we write

$$
f_{i_{0} \ldots i_{q}}(p)=\sum_{\lambda=1}^{\operatorname{rank} E} \sum_{|Q|=L} f_{i_{0} \ldots i_{q} ; Q}^{\lambda}\left(z_{i_{q}}(p)\right) e_{i_{0}, \lambda}(p) \otimes\left(w_{i_{q}}^{*}(p)\right)^{Q},
$$


where $e_{i_{0}}$ is the base of $E$ over $U_{i_{0}}$ and $w_{i_{q}}^{*}$ is the base of $N_{C}^{*}$ on $U_{i_{q}}$. Define

$$
|f|_{\mathrm{mf}, \mathcal{U}}^{2}:=\max _{\left(i_{0}, \ldots, i_{q}\right) \in \mathcal{I}^{q+1}} \sup _{z_{i_{q}} \in \varphi_{i_{q}}\left(U_{i_{0} \ldots i_{q}}\right)} \sum_{\lambda=1}^{\operatorname{rank} E} \sum_{Q} \frac{Q !}{|Q| !}\left|f_{i_{0} \cdots i_{q} ; Q}^{\lambda}\left(z_{i_{q}}\right)\right|^{2} .
$$

When there is no confusion, we shall in the sequel write "f" instead of " $m f$ ". The following two propositions are a "version with parameters" of [28, propositions 3.6-3.7] (see also [23]). We only give the proof of the last two points of next proposition.

Proposition 3.4. Let $\mathcal{O}_{n}(\omega) \otimes \mathbb{C}\left[x_{1}, \ldots, x_{d}\right]$ be the set of polynomials $f(x, z)$ in $x$ with coefficients holomorphic in $z \in \omega \subset \mathbb{C}^{n}$.

(a) Let $f, g \in \mathcal{O}_{n}(\omega) \otimes \mathbb{C}\left[x_{1}, \ldots, x_{d}\right]$ be homogeneous polynomials of degree $k, k^{\prime}$ respectively. Then

$$
|f g|_{f, \omega} \leq|f|_{f, \omega}|g|_{f, \omega} .
$$

(b) Let $f \in \mathcal{O}_{n}(\omega) \otimes \mathbb{C}\left[x_{1}, \ldots, x_{d}\right]$ and let $\tilde{f}_{P}(z, x)=\frac{1}{P !} \partial_{z}^{P} f(z, x)$. Then

$$
\left|\tilde{f}_{P}\right|_{f, \omega^{\prime}} \leq \frac{|f|_{f, \omega}}{\left(\operatorname{dist}_{*}\left(\omega^{\prime}, \partial \omega\right)\right)^{|P|}}, \quad \forall \omega^{\prime} \subset \omega, \operatorname{dist}_{*}\left(\omega^{\prime}, \partial \omega\right):=\operatorname{dist}\left(\omega^{\prime}, \partial \omega\right) / \sqrt{n} .
$$

(c) Let $T$ be a $d \times d$ unitary matrix. Let $f \in \mathcal{O}_{n}^{d}(\omega) \otimes \mathbb{C}\left[x_{1}, \ldots, x_{d}\right]$. Then,

$$
|T f|_{f, \omega}=|f|_{f, \omega}
$$

(d) Let $T$ be a $d \times d$ unitary matrix. Let $f \in \mathcal{O}_{n}(\omega) \otimes \mathbb{C}\left[x_{1}, \ldots, x_{d}\right]$ and $f^{T}(z, x):=$ $f(z, T x)$. Then,

$$
\left|f^{T}\right|_{f, \omega}=|f|_{f, \omega}
$$

Proof. We only prove the last two points. Fix $z \in \omega^{\prime}$. The polydisc center at $z$ with radius $\delta:=\operatorname{dist}\left(\omega^{\prime}, \partial \omega\right) / \sqrt{n}$ is contained in $\omega$.

By the Cauchy formula, we have

$$
\begin{aligned}
\tilde{f}_{P}(z, x) & =\frac{1}{\delta^{|P|}} \int_{[0,2 \pi]^{n}} f\left(z+\delta\left(e^{i \theta_{1}}, \ldots, e^{i \theta_{n}}\right), x\right)\left(e^{i \theta_{1}}, \ldots, e^{i \theta_{n}}\right)^{-P} \frac{d \theta_{1}}{2 \pi} \cdots \frac{d \theta_{n}}{2 \pi} \\
& =\frac{1}{\delta^{|P|}} \sum_{Q \in \mathbf{N}^{d}} x^{Q} \int_{[0,2 \pi]^{n}} f_{Q}\left(z+\delta\left(e^{i \theta_{1}}, \ldots, e^{i \theta_{n}}\right)\right)\left(e^{i \theta_{1}}, \ldots, e^{i \theta_{n}}\right)^{-P} \frac{d \theta_{1}}{2 \pi} \cdots \frac{d \theta_{n}}{2 \pi} .
\end{aligned}
$$

We emphasize that the sum is finite. By the Cauchy-Schwarz inequality applied to the integral, we have

$$
\begin{aligned}
\left|\tilde{f}_{P}(z, \cdot)\right|_{\mathrm{mf}}^{2} & =\frac{1}{\delta^{2|P|}} \sum_{Q \in \mathbf{N}^{d}}\left|x^{Q}\right|_{\mathrm{mf}}^{2}\left|\int_{[0,2 \pi]^{n}} f_{Q}\left(z+\delta\left(e^{i \theta_{1}}, \ldots, e^{i \theta_{n}}\right)\right)\left(e^{i \theta_{1}}, \ldots, e^{i \theta_{n}}\right)^{-P} \frac{d \theta_{1}}{2 \pi} \cdots \frac{d \theta_{n}}{2 \pi}\right|^{2} \\
& \leq \frac{1}{\delta^{2|P|}} \sum_{Q \in \mathbf{N}^{d}}\left|x^{Q}\right|_{\mathrm{mf}}^{2} \int_{[0,2 \pi]^{n}}\left|f_{Q}\left(z+\delta\left(e^{i \theta_{1}}, \ldots, e^{i \theta_{n}}\right)\right)\right|^{2} \frac{d \theta_{1}}{2 \pi} \cdots \frac{d \theta_{n}}{2 \pi} \\
& =\frac{1}{\delta^{2|P|}} \int_{[0,2 \pi]^{n}} \sum_{Q \in \mathbf{N}^{d}}\left|x^{Q}\right|_{\mathrm{mf}}^{2}\left|f_{Q}\left(z+\delta\left(e^{i \theta_{1}}, \ldots, e^{i \theta_{n}}\right)\right)\right|^{2} \frac{d \theta_{1}}{2 \pi} \cdots \frac{d \theta_{n}}{2 \pi} \\
& \leq \frac{1}{\delta^{2|P|}} \int_{[0,2 \pi]^{n}}|f|_{\omega}^{2} \frac{d \theta_{1}}{2 \pi} \cdots \frac{d \theta_{n}}{2 \pi}=\frac{1}{\delta^{2|P|}}|f|_{\omega}^{2} .
\end{aligned}
$$


For the last point, we have, for a homogeneous polynomial $f$ in $x$ of degree $m$ with holomorphic coefficients in $\omega$ the identity:

$$
\left|f_{m}\right|_{\omega}^{2}=\frac{1}{\pi^{d} m !} \sup _{z \in \omega} \int_{\mathbb{C}^{d}}|f(z, x)|^{2} e^{-|x|^{2}} d V(x) .
$$

In particular, the integral is invariant under the transformation $x \rightarrow T x$ when $T$ is unitary (and constant).

Proposition 3.5. For a formal power series $f(h, v)=\sum_{k} f_{k}(z, v)$ with $f_{k}(z, v)$ being a homogeneous polynomial in $v$ of degree $k$ of which the coefficients are functions holomorphic in $z \in U$, the following properties are equivalent:

(a) $f$ is uniformly convergent for $v$ in a neighborhood of the origin, uniformly in $U$.

(b) There exist $M, R>0$ such that for every $k,\left|f_{k}\right|_{m f, U} \leq \frac{M}{R^{k}}$.

For convenience, we will use the following orthonormal Fischer base of $S^{L} N_{C}^{*}$ :

$$
e_{j, Q}^{*}=\sqrt{\frac{|Q| !}{Q !}}\left(w_{j}^{*}\right)^{Q}, \quad|Q|=L, \quad Q \in \mathbf{N}^{d} .
$$

The transition matrices $t_{k j}^{L}$ of $S^{L} N_{C}^{*}$ is then determined in the following way : Let $F_{k}=$ $\sum_{|P|=L} F_{k, P} e_{k, P}^{*}$. We have

$$
\left(F_{k, P}\right)_{|P|=L}=t_{k j}^{L}\left(F_{j, P}\right)_{|P|=L} .
$$

This can be computed from the transition matrices of $N_{C}^{*}$ by expressing the basis $w_{k, 1}^{*}, \ldots, w_{k, d}^{*}$ in terms of $w_{j, 1}^{*}, \ldots, w_{j, d}^{*}$. Since $t_{k j}^{L}$ maps orthonormal basis into orthonormal basis, by Proposition 3.4 we know that $t_{k j}^{L}$ are unitary matrices, i.e. in operator norm defined in (A.4),

$$
\left|t_{k j}^{L}\right|=1, \quad L=1,2, \ldots
$$

We will apply results in the appendix to the transition matrices $t_{k j}^{L}$.

\subsection{A majorization in the modified Fischer norm for the vertical linearization.}

Let $\left\{f_{j}^{v}\right\}$ be the formal solution of (3.2). We use notation (3.7). Let $\varphi_{j}\left(U_{j}\right)=\Delta_{n}$ and $U_{k j}:=U_{k} \cap U_{j}$. Define $\hat{U}_{k j}=\varphi_{j}\left(U_{k j}\right)$. Then, $\varphi_{k j}\left(\hat{U}_{k j}\right)=\hat{U}_{j k}$. Let us first assume that $H^{0}\left(C, N_{C} \otimes S^{\ell}\left(N_{C}^{*}\right)\right)=0$ for all $\ell \geq 2$. We shall see later on how to get rid of this assumption to prove the general result.

Let us assume that there exists a vertical formal transformation $F:=\left\{F_{j}\right\}$ fixing $C$, being tangent to identity on it, and preserving the splitting of $T_{C} M$ that linearizes vertically a neighborhood of $C$ in $M$. Let us write

$$
F_{j}\left(h_{j}, v_{j}\right):=\left(h_{j}, v_{j}+f_{j}\right), \quad f_{j}=\sum_{k \geq 2}\left[f_{j}\right]^{k}, \quad\left\{\left[f_{j}\right]^{k}\right\} \in C^{0}\left(C, N_{C} \otimes S^{k}\left(N_{C}^{*}\right)\right) .
$$

Assume that there is a sequence $\left\{A_{k}\right\}_{k \geq 2}$ of positive numbers such that

$$
\forall k<m \quad\left|\left[f_{j}\right]^{k}\right|_{\hat{U}_{j}} \leq \eta_{k} A_{k} .
$$

Let us set

$$
A(t)=\sum_{k \geq 2} A_{k} t^{k}
$$


with $t \in \mathbb{C}$. Let us first estimate both $\left|\left[h_{k j}^{\prime}\right]^{m}\right|_{\hat{U}_{k j}}$ and $\left|\left[h_{k j}^{\prime \prime}\right]^{m}\right|_{\hat{U}_{k j}}$ in term of $J^{m-1} A(t):=$ $A_{2} t^{2}+\cdots+A_{m-1} t^{m-1}$.

Since $\phi_{k j}^{h}$ is holomorphic in $h_{j} \in \hat{U}_{k j}$ and $v_{j}$ in a neighborhood of the origin. We can assume that there is a positive $R$ such that

$$
\sup _{h_{j} \in \hat{U}_{k j}}\left|\phi_{k j, Q}^{h}\left(h_{j}\right)\right| \leq R^{|Q|}
$$

for all $Q \in \mathbb{N}_{2}^{d}$, where $\phi_{k j, Q}^{h}$ is defined by (3.3) and $\mathbb{N}_{k}^{d}:=\left\{Q \in \mathbb{N}^{d}:|Q| \geq k\right\}$.

For $Q \in \mathbb{N}_{2}^{d}$, we have

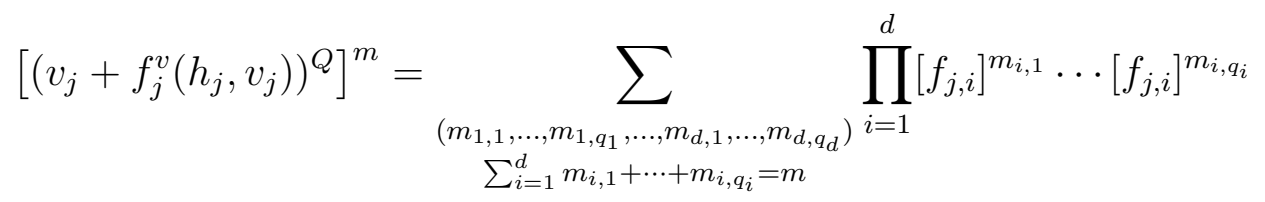

where we have set $f_{j}^{v}=\left(f_{j, 1}, \ldots, f_{j, d}\right),\left[f_{j, i}\right]^{1}=v_{j, i}$ and $\left[f_{j, i}\right]^{0}=0$. In the following, all $m_{i, j}$ are positive integers. Hence, by the first point of Proposition 3.4, we have $(3.10)$

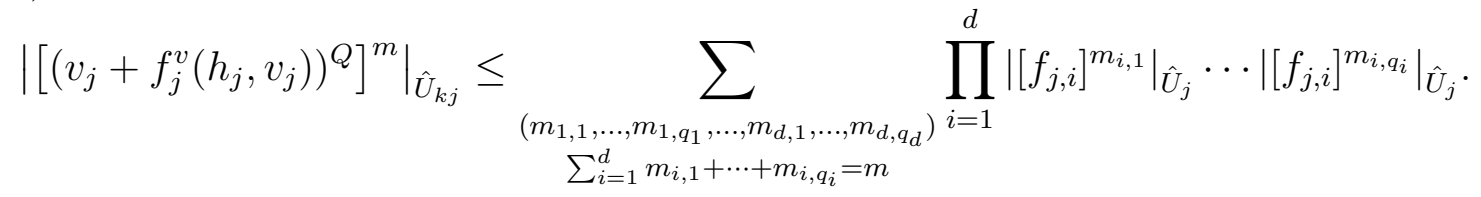

Let $m \geq 2$, for $Q \in \mathbb{N}_{2}^{d},|Q| \leq m$, let us set

$$
E_{Q, m}=\left\{\left(m_{1,1}, \ldots, m_{1, q_{1}}, \ldots, m_{d, 1}, \ldots, m_{d, q_{d}}\right) \in \mathbb{N}_{1}^{|Q|}: \sum_{i=1}^{d} m_{i, 1}+\cdots+m_{i, q_{i}}=m\right\}
$$

Let $M_{i}=\left(m_{1,1}^{(i)}, \ldots, m_{1, q_{1}^{(i)}}^{(i)}, \ldots, m_{d, 1}^{(i)}, \ldots, m_{d, q_{d}^{(i)}}^{(i)}\right) \in \mathbb{N}_{1}^{\left|Q^{(i)}\right|}$ with $\left|Q^{(i)}\right| \leq m_{i}$ and $m_{i}=$ $\sum_{j=1}^{d} m_{j, 1}^{(i)}+\cdots+m_{j, q_{j}^{(i)}}^{(i)}, i=1,2$. Define the concatenation $M_{1} \sqcup M_{2}$ to be $\left(M_{1}, M_{2}\right)$. We also have $\sum_{j=1}^{2} \sum_{i=1}^{d} m_{i, 1}^{(j)}+\cdots+m_{i, q_{i}^{(j)}}^{(j)}=m_{1}+m_{2}$. Hence, we emphasize that the concatenation

$$
\left(\bigcup_{2 \leq\left|Q_{1}\right| \leq m_{1}} E_{Q_{1}, m_{1}}\right) \sqcup\left(\bigcup_{2 \leq\left|Q_{2}\right| \leq m_{2}} E_{Q_{2}, m_{2}}\right) \subset \bigcup_{2 \leq|Q| \leq m_{1}+m_{2}} E_{Q, m_{1}+m_{2}} .
$$


As a consequence, according to (3.3) and (4.4), we have

$$
\begin{aligned}
\left|\left[\sum_{Q \in \mathbb{N}^{d},|Q|=m} h_{k j, Q}^{\prime}\left(h_{j}\right) v_{j}^{Q}\right]^{m}\right| & \leq \sum_{|Q|=2}^{m} R^{|Q|} \sum_{M \in E_{Q, m}} \prod_{i=1}^{d}\left|\left[f_{j, i}\right]^{m_{i, 1}}\right|_{\hat{U}_{j}} \cdots\left|\left[f_{j, i}\right]^{m_{i, q_{i}}}\right|_{\hat{U}_{j}} \\
& \leq \sum_{|Q|=2}^{m} R^{|Q|} \sum_{M \in E_{Q, m}} \prod_{i=1}^{d} \eta_{m_{i, 1}} A_{m_{i, 1}} \cdots \eta_{m_{i, q_{i}}} A_{m_{i, q_{i}}} \\
& \leq\left[\sum_{|Q|=2}^{m} \eta_{Q, m} R^{|Q|}\left(t+J^{m-1}(A(t))^{|Q|}\right]^{m}\right. \\
& \leq E_{m}\left[g_{m}(t)\right]^{m},
\end{aligned}
$$

where we have set

$$
\begin{aligned}
& \eta_{Q, m}:=\max _{M \in E_{Q, m}}\left(\prod_{i=1}^{d} \eta_{m_{i, 1}} \cdots \eta_{m_{i, q_{i}}}\right), \quad E_{m}:=\max _{\substack{Q \in \mathbb{N}^{d} \\
2 \leq|Q| \leq m}} \eta_{Q, m}, \\
& g_{m}(t):=\sum_{|Q|=2}^{m} R^{|Q|}\left(t+J^{m-1}(A(t))^{|Q|}, \quad g(t):=\sum_{|Q| \geq 2} R^{|Q|}(t+A(t))^{|Q|} .\right.
\end{aligned}
$$

Hence, as formal power series, we have

$$
g(t)=\left(\frac{1}{1-R(t+A(t))}\right)^{d}-d R(t+A(t))-1
$$

Let $\mathcal{U}^{*}=\left\{U_{i}^{*}\right\}$ be an open covering of $C$ such that $U_{i}^{*}$ is relatively compact in $U_{i}$. We shall write $\hat{U}_{k}^{*}:=\varphi_{k}\left(U_{k}^{*}\right)$. Let us consider the index $j$ as fixed and let us estimate the Fischer norm of $h_{k j}^{\prime \prime}$ on $\hat{U}_{k j}^{*}:=\varphi_{j}\left(U_{j} \cap U_{k}^{*}\right)$. We have

$$
\begin{aligned}
{\left[\sum_{Q \in \mathbb{N}^{d},|Q|=m} h_{k j, Q}^{\prime \prime}\left(h_{j}\right) v_{j}^{Q}\right]^{m} } & =\sum_{\substack{P \in \mathbb{N}_{1}^{n} \\
m_{1}+m_{2}=m}} \frac{1}{P !}\left[\partial_{h}^{P} f_{k}\left(\varphi_{k j}\left(h_{j}\right), t_{k j} v_{j}\right)\right]^{m_{1}}\left[\left(\phi_{k j}^{h}\left(h_{j}, v_{j}+f_{j}^{v}\right)\right)^{P}\right]^{m_{2}} \\
& =\sum_{\substack{P \in \mathbb{N}_{1}^{n} \\
m_{1}+m_{2}=m}} \frac{1}{P !}\left[\partial_{h}^{P} f_{k}\left(\varphi_{k j}\left(h_{j}\right), t_{k j} v_{j}\right)\right]^{m_{1}}\left[\left(h_{k j}^{\prime}\left(h_{j}, v_{j}\right)\right)^{P}\right]^{m_{2}} .
\end{aligned}
$$

Here, both indices $m_{1}$ and $m_{2}$ are $\geq 2$. Since the Fischer norm is submultiplicative, we have

$$
\left|\left[\left(h_{k j}^{\prime}\left(h_{j}, v_{j}\right)\right)^{P}\right]^{m_{2}}\right|_{\hat{U}_{k j}^{*}} \leq E_{m_{2}}\left[\left(\sum_{|Q|=2}^{\frac{m}{2}} R^{|Q|}\left(t+J^{m-1}(A(t))^{|Q|}\right)^{|P|}\right]^{m_{2}}\right.
$$


Indeed,

$$
\begin{aligned}
{\left[\left(h_{k j}^{\prime}\left(h_{j}, v_{j}\right)\right)^{P}\right]^{m_{2}} } & =\left[\prod_{i=1}^{n}\left(h_{k j, i}^{\prime}\right)^{p_{i}}\right]^{m_{2}} \\
& =\sum_{\sum_{i}\left(m_{i, 1}+\cdots+m_{i, p_{i}}\right)=m_{2}} \prod_{i=1}^{n}\left[h_{k j, i}^{\prime}\right]^{m_{i, 1}} \cdots\left[h_{k j, i}^{\prime}\right]^{m_{i, p_{i}}} .
\end{aligned}
$$

According to (3.11) and by (3.12), we have

$$
\begin{aligned}
\left|\prod_{i=1}^{n}\left[h_{k j, i}^{\prime}\right]^{m_{i, 1}} \cdots\left[h_{k j, i}^{\prime}\right]^{m_{i, p_{i}}}\right|_{\hat{U}_{k j}^{*}} & \leq \prod_{i=1}^{n} E_{m_{i, 1}}\left[g_{m_{i, 1}}(t)\right]^{m_{i, 1}} \cdots E_{m_{i, p_{i}}}\left[g_{m_{i, p_{i}}}(t)\right]^{m_{i, p_{i}}} \\
& \leq \max _{2 \leq|Q| \leq m_{2}} \eta_{Q, m_{2}} \prod_{i=1}^{n}\left[g_{m_{i, 1}}(t)\right]^{m_{i, 1}} \cdots\left[g_{m_{i, p_{i}}}(t)\right]^{m_{i, p_{i}}} .
\end{aligned}
$$

Hence, we have

$$
\sum_{\sum_{i}\left(m_{i, 1}+\cdots+m_{i, p_{i}}\right)=m_{2}}\left|\prod_{i=1}^{n}\left[h_{k j, i}^{\prime}\right]^{m_{i, 1}} \cdots\left[h_{k j, i}^{\prime}\right]^{m_{i, p_{i}}}\right|_{\hat{U}_{k j}^{*}} \leq E_{m_{2}}\left[g(t)^{|P|}\right]^{m_{2}} .
$$

We have, by definition $\left[\partial_{h}^{P} f_{k}\left(\varphi_{k j}\left(h_{j}\right), t_{k j} v_{j}\right)\right]^{m_{1}}=\partial_{h}^{P}\left[f_{k}\right]^{m_{1}}\left(\varphi_{k j}\left(h_{j}\right), t_{k j} v_{j}\right)$.

Recall that the Fischer norm is unitary invariant and by Proposition 3.4, we have

$$
\begin{aligned}
\left|\partial_{h}^{P}\left[f_{k}\right]^{m_{1}}\left(\varphi_{k j}\left(h_{j}\right), t_{k j} v_{j}\right)\right|_{\hat{U}_{k j}^{*}}^{2} & =\left|\partial_{h}^{P}\left[f_{k}\right]^{m_{1}}\left(\varphi_{k j}\left(h_{j}\right), v_{j}\right)\right|_{\hat{U}_{k j}^{*}}^{2} \\
& \leq\left(\frac{P !}{\operatorname{dist}_{*}\left(\hat{U}_{k}^{*}, \partial \hat{U}_{k}\right)^{|P|}}\right)^{2}\left|\left[f_{k}\right]^{m_{1}}\right|_{\hat{U}_{k}}^{2} .
\end{aligned}
$$

Let us set $M:=\inf _{k} \operatorname{dist}\left(\hat{U}_{k}^{*}, \partial \hat{U}_{k}\right)$. As a consequence, we have

$$
\begin{aligned}
\left|\left[\sum_{Q \in \mathbb{N}^{d},|Q|=m} h_{k j, Q}^{\prime \prime}\left(h_{j}\right) v_{j}^{Q}\right]^{m}\right|_{\hat{U}_{k j}^{*}} & \leq \sum_{m_{1}+m_{2}=m} \sum_{\substack{P \in \mathbb{N}^{n} \\
|P| \geq 1}} \frac{1}{M^{|P|} \mid}\left|\left[f_{k}\right]^{m_{1}}\right|_{\hat{U}_{k}} E_{m_{2}}\left[g(t)^{|P|}\right]^{m_{2}} \\
& \leq \sum_{m_{1}+m_{2}=m}\left|\left[f_{k}\right]^{m_{1}}\right|_{\hat{U}_{k}}\left[E_{m_{2}} \sum_{\substack{P \in \mathbb{N}^{n} \\
|P| \geq 1}}\left(\frac{g(t)}{M}\right)^{|P|}\right]^{m_{2}} \\
& \leq\left(\max _{m_{1}+m_{2}=m} \eta_{m_{1}} E_{m_{2}}\right)\left[A(t)\left(\left(\frac{M}{M-g(t)}\right)^{n}-1\right)\right]^{m} .
\end{aligned}
$$

Collecting estimates (3.12) and (3.14), we obtain

$$
\left|\mathcal{L}_{k j}^{v}\left(\left[f_{j}^{v}\right]^{m}\right)\right|_{\hat{U}_{k j}^{*}} \leq\left[E_{m} g(t)+\left(\max _{m_{1}+m_{2}=m} \eta_{m_{1}} E_{m_{2}}\right) A(t)\left(\left(\frac{M}{M-g(t)}\right)^{n}-1\right)\right]^{m} .
$$

Let us extend this to an estimate on $\hat{U}_{k j}=\varphi_{j}\left(U_{j} \cap U_{k}\right)$. Following again Ueda's argument [40] let us express the fact that $[h]^{m}:=\left[h^{\prime}\right]^{m}+\left[h^{\prime \prime}\right]^{m}$ is a 1 -cocycle with values in 
$N_{C} \otimes S^{m}\left(N_{C}^{*}\right)$. Let $p \in U_{k} \cap U_{j}$. Then $p \in U_{k} \cap U_{j} \cap U_{i}^{*}$ for some $i$. According to (3.4) and Lemma 2.7, at $p \in U_{k} \cap U_{j} \cap U_{i}^{*}$ we have

$$
t_{k i} \sum_{|Q|=m} h_{i k, Q}\left(z_{k}(p)\right)\left(t_{k j} v_{j}\right)^{Q}-t_{k i} \sum_{|Q|=m} h_{i j, Q}\left(z_{j}(p)\right)\left(v_{j}\right)^{Q}+\sum_{|Q|=m} h_{k j, Q}\left(z_{j}(p)\right)\left(v_{j}\right)^{Q}=0 .
$$

Here by (3.7) the Fischer norms of $h_{k j}$ on all subdomains must be computed in the base $e_{k}^{v}$ of $N_{C}$ on $U_{k}$ and the base $w_{j}^{*}$ of $N_{C}^{*}$ on $U_{j}$. We can apply the previous estimates (3.12) and (3.14) to the first two sums respectively on $\hat{U}_{i k}^{*}$ and $\hat{U}_{i j}^{*}$. To estimate the first sum, we need to change coordinates. From section 2, $t_{k j}$ (resp. $s_{k j}$ ) are transition matrices of $N_{C}$ (resp. TC). Recall that $\left\{\left[h_{k j}\right]^{m}\right\} \in Z^{1}\left(\mathcal{U}^{r_{*}}, N_{C} \otimes S^{m} N_{C}^{*}\right)$ and

$$
\begin{aligned}
h_{i k}(p) & =\sum_{\lambda=1}^{d} \sum_{|Q|=m} h_{i k ; Q}^{\lambda}\left(z_{k}(p)\right) e_{i, \lambda}^{v}(p) \otimes\left(w_{k}^{*}(p)\right)^{Q} \\
& =\sum_{\lambda^{\prime}=1}^{d} \sum_{\lambda=1}^{d} \sum_{|Q|=m} h_{i k ; Q}^{\lambda}\left(z_{k}(p)\right) t_{k i, \lambda}^{\lambda^{\prime}}\left(z_{k}(p)\right) e_{k, \lambda^{\prime}}^{v}(p) \otimes\left(t_{k j} w_{j}^{*}(p)\right)^{Q}=: \tilde{h}_{k j}\left(z_{k}(p), w_{j}^{*}\right) .
\end{aligned}
$$

Thus, $\sum_{|Q|=m} h_{i k, Q}\left(z_{k}(p)\right)\left(t_{k j} v_{j}\right)^{Q}=\tilde{h}_{k j}\left(z_{k}(p), v_{j}\right)$. By the unitary invariance by multiplication and composition of the Fischer norm and by definition (3.7), we have for fixed $z_{k}(p) \in \hat{U}_{i k}^{*}$,

$$
\begin{aligned}
\left|\tilde{h}_{k j}\left(z_{k}(p), v_{j}\right)\right|_{\mathrm{mf}}^{2} & =\sum_{\lambda^{\prime}=1}^{d}\left|\sum_{|Q|=m}\left(\sum_{\lambda=1}^{d} t_{k i, \lambda}^{\lambda^{\prime}}\left(z_{k}\right) h_{i k ; Q}^{\lambda}\left(z_{k}\right)\right)\left(t_{k j} v_{j}\right)^{Q}\right|_{\mathrm{mf}}^{2} \\
& =\sum_{\lambda^{\prime}=1}^{d}\left|\sum_{|Q|=m}\left(\sum_{\lambda=1}^{d} t_{k i, \lambda}^{\lambda^{\prime}}\left(z_{k}\right) h_{i k ; Q}^{\lambda}\left(z_{k}\right)\right) v_{j}^{Q}\right|_{\mathrm{mf}}^{2} \\
& =\sum_{\lambda^{\prime}=1}^{d} \sum_{|Q|=m} \frac{Q !}{|Q| !}\left|\sum_{\lambda=1}^{d} t_{k i, \lambda}^{\lambda^{\prime}}\left(z_{k}\right) h_{i k ; Q}^{\lambda}\left(z_{k}\right)\right|^{2} \\
& \leq \sum_{\lambda^{\prime}} \sum_{|Q|=m} \frac{Q !}{|Q| !} \sum_{\lambda=1}^{d}\left|h_{i k ; Q}^{\lambda}\left(z_{k}\right)\right|^{2} \leq d\left|h_{i k}\right|_{\hat{U}_{i k}^{*}}^{2}
\end{aligned}
$$

where the second last inequality is obtained by the Cauchy-Schwarz inequality. In a similar way, we have a similar estimate for the second sum in (3.15) on $\varphi_{j}\left(U_{k} \cap U_{j} \cap U_{i}^{*}\right)$. For the third sum in (3.15), we note that the entries of the unitary matrix $t_{k i}$ have modulus at most one. Thus, there exist constants $M^{\prime}, \tilde{M}$ such that the third sum in (3.15) satisfies

$$
\begin{aligned}
\left|h_{k j}\right|_{\hat{U}_{k j}} & \leq M^{\prime} \max _{i}\left(\left|h_{i k}\right|_{\hat{U}_{i k}^{*}}+\left|h_{i j}\right|_{\hat{U}_{i j}^{*}}\right) \\
& \leq \tilde{M} \max \left(E_{m}, \max _{\substack{m_{1}+m_{2}=m \\
m_{1}, m_{2} \geq 2}} \eta_{m_{1}} E_{m_{2}}\right)\left[g(t)+A(t)\left(\left(\frac{M}{M-g(t)}\right)^{n}-1\right)\right]^{m} .
\end{aligned}
$$


We now adapt the estimate in Lemma A.2 (see also Theorem A.12). Recall that $\left[h_{k j}\right] \leq m$ depends only on $[f]^{\leq m-1}$ and the hypothesis (3.9). By the formal assumption, we have a solution to $(3.4)$ :

$$
\mathcal{L}_{k j}\left(\left[f_{j}^{v}\right]^{m}\right)=\left[h_{k j}\right]^{m} .
$$

By assumptions, $H^{0}\left(C, N_{C} \otimes S^{\ell}\left(N_{C}^{*}\right)\right)=0$, for all $\ell \geq 2$. Hence, the solution of the previous equation is unique. By Lemma A.2, (A.5) and (3.8), the solution satisfies the estimate:

$$
\left|\left\{\left[f_{j}^{v}\right]^{m}\right\}\right|_{\mathcal{U}} \leq\left. C\left(1+K_{*}\left(N_{C} \otimes S^{m} N_{C}^{*}\right) \mid\right)\left\{\left[h_{k j}\right]^{m}\right\}\right|_{\mathcal{U}} .
$$

Here, $C$ depends neither on $N_{C}$ nor on $S^{m} N_{C}^{*}$. Therefore, we have

$$
\left|\left[f_{j}^{v}\right]^{m}\right|_{\hat{U}_{j}} \leq K\left(N_{C} \otimes S^{m}\left(N_{C}^{*}\right)\right) \max _{k}\left|\mathcal{L}_{k j}^{v}\left(\left[f_{j}^{v}\right]^{m}\right)\right|_{\hat{U}_{k j}^{*}} .
$$

By definition (3.1), we have

$$
K\left(N_{C} \otimes S^{m}\left(N_{C}^{*}\right)\right) \max \left(E_{m}, \max _{\substack{m_{1}+m_{2}=m \\ m_{1}, m_{2} \geq 2}} \eta_{m_{1}} E_{m_{2}}\right) \leq \eta_{m} .
$$

Hence, we have

$$
\left|\left[\left\{f^{v}\right\}\right]^{m}\right|_{\hat{U}} \leq \tilde{M} \eta_{m}\left[g(t)+A(t)\left(\left(\frac{M}{M-g(t)}\right)^{n}-1\right)\right]^{m} .
$$

Let us consider the functional equation

$$
A(t)=\mathcal{F}(t, A(t)):=\tilde{M}\left(g(t)+A(t)\left(\left(\frac{M}{M-g(t)}\right)^{n}-1\right)\right),
$$

where $g(t)$ is a function of $A$ by (3.13). This equation has a unique analytic solution vanishing at the origin at order 2 .

We now can prove the theorem. Indeed by assumption, there are positive constants $M, L$ such that $\eta_{m} \leq M L^{m}$ for all $m \geq 2$. Since $A(t)$ converges at the origin, then $A_{m} \leq D^{m}$ for some positive $D$. According to (3.16), we have also proved

$$
\left|\left[\left\{f^{v}\right\}\right]^{m}\right|_{\hat{U}} \leq \eta_{m} A_{m},
$$

so that, finally, $\left|\left[\left\{f^{v}\right\}\right]^{m}\right|_{\hat{U}} \leq M(D L)^{m}$ for all $m \geq 2$. Hence, $f^{v}=\sum_{m \geq 2}\left[\left\{f^{v}\right\}\right]^{m}$ converges at the origin and this proves the theorem.

Let us see how we can prove Remark 3.2. The issue is that, when considering a solution $\left[f_{j}^{v}\right]^{m}$ of the cohomological equation $\mathcal{L}_{k j}\left(\left[f_{j}^{v}\right]^{m}\right)=R^{m}$, the estimate given by Lemma A.2 and Proposition A.4 might be obtained by another solution. Hence, the formal solution might not be the good one for the estimate. Furthermore, we cannot replace a solution at degree $m$ as we wish to ensure that higher order terms in the vertical component can be eliminated formally. We now explain the general result as formulated in the theorem. We will assume that there are formal mappings

$$
\tilde{F}_{j}\left(h_{j}, v_{j}\right)=\left(h_{j}, v_{j}\right)+\left(0, \sum_{\ell>2} \tilde{f}_{j, \ell}^{v}\left(h_{j}, v_{j}\right)\right)
$$

satisfying the following 
(1) $\left\{\tilde{F}_{k}^{-1} \Phi_{k j} \tilde{F}_{j}-N_{k j}\right\}^{v}=0$ for all $k, j$. In other words, $\left\{\tilde{F}_{j}\right\}$ formally linearizes $\Phi_{k j}$ vertically. In particular,

$$
\left\{\left(\tilde{F}_{k}^{m}\right)^{-1} \Phi_{k j} \tilde{F}_{j}^{m}-N_{k j}\right\}^{v}=\left[\phi_{k j}^{v}\right]^{m}+R_{k j}^{m}\left(\left\{\left[\phi_{k j}\right]^{\ell},\left[\tilde{f}_{k}^{v}\right]^{\ell}\right\}_{2 \leq \ell<m}\right)+O\left(\left|v_{j}\right|^{m+1}\right)
$$

for

$$
\tilde{F}_{j}^{m}=\left(h_{j}, v_{j}\right)+\left(0, \sum_{2 \leq \ell \leq m} \tilde{f}_{j, \ell}^{v}\left(h_{j}, v_{j}\right)\right) .
$$

(The last assertion can be check easily since $\left.\left(\tilde{F}_{j}^{m}\right)^{-1} \tilde{F}_{j}\left(h_{j}, v_{j}\right)=\left(h_{j}, v_{j}\right)+O\left(\left|v_{j}\right|^{m+1}\right)\right)$.

(2) Each $\left\{\tilde{f}_{j, m}^{v}\right\}_{j}$ is a "minimizer" in the sense that it satisfies the equation

$$
\left\{\delta^{v} \tilde{f}_{m}^{v}\right\}_{k j}=\left[\phi_{k j}^{v}\right]^{m}+\left[R^{m}\left(\left\{\left[\phi_{k j}\right]^{\ell},\left[\tilde{f}_{k}^{v}\right]^{\ell}\right\}_{2 \leq \ell<m}\right)\right]^{m}
$$

and the estimate

$$
\left|\tilde{f}_{m}^{v}\right| \leq K\left(N_{C} \otimes S^{m}\left(N_{C}^{*}\right)\right)\left|\left[\phi^{v}\right]^{m}+\left[R_{k j}^{m}\left(\left\{\left[\phi_{k j}\right]^{\ell},\left[\tilde{f}_{k}^{v}\right]^{\ell}\right\}_{2 \leq \ell<m}\right)\right]^{m}\right| .
$$

As a consequence, the scheme of convergence applies to that formal solution $\left\{\tilde{F}_{j}\right\}$ and we are done.

\section{A majorant method For the FUlL LineARizATion With a UNitARY NORMAL BUNDLE}

In this section, we shall devise a proof of Theorem 1.4, that is of the linearization of the neighborhood problem in the case $N_{C}$ is unitary (and flat) following a majorant method scheme.

Let us recall the horizontal cohomological operator

$$
\mathcal{L}_{k j}^{h}\left(f_{j}^{h}\right):=f_{k}^{h}\left(\varphi_{k j}\left(h_{j}\right), t_{k j} v_{j}\right)-s_{k j}\left(h_{j}\right) f_{j}^{h}\left(h_{j}, v_{j}\right)
$$

where $s_{k j}\left(h_{j}\right)=D \varphi_{k j}\left(h_{j}\right)$. We then have the horizontal equation (2.17)

$$
\begin{aligned}
\mathcal{L}_{k j}^{h}\left(f_{j}^{h}\right)= & \phi_{k j}^{h}\left(h_{j}+f_{j}^{h}, v_{j}+f_{j}^{v}\right) \\
& +\varphi_{k j}\left(h_{j}+f_{j}^{h}\left(h_{j}, v_{j}\right)\right)-\varphi_{k j}\left(h_{j}\right)-D \varphi_{k j}\left(h_{j}\right) f_{j}^{h}\left(h_{j}, v_{j}\right) .
\end{aligned}
$$

Let us recall the vertical cohomological operator

$$
\mathcal{L}_{k j}^{v}\left(f_{j}^{v}\right):=f_{k}^{v}\left(\varphi_{k j}\left(h_{j}\right), t_{k j} v_{j}\right)-t_{k j} f_{j}^{v},
$$

and vertical equation (2.19) (recall that $N_{C}$ is flat)

$$
\mathcal{L}_{k j}^{v}\left(f_{j}^{v}\right)=\varphi_{k j}^{v}\left(h_{j}+f_{j}^{h}, v_{j}+f_{j}^{v}\right) .
$$

By assumption, there exists a formal solution $f_{j}=\left(f_{j}^{h}, f_{j}^{v}\right)=\sum_{k \geq 2}\left[f_{j}\right]^{k}$ with $\left\{\left[f_{j}\right]^{k}\right\} \in$ $C^{0}\left(C, T_{C} M \otimes S^{k}\left(N_{C}^{*}\right)\right)$. In case we assume $H^{1}\left(C, T_{C} M \otimes S^{k}\left(N_{C}^{*}\right)\right)=0$, for all $k \geq 2$, this follows from Lemma 2.10. We now use the "norm" of the cohomological operator acting on $C^{0}\left(\mathcal{U}, T_{C} M \otimes S^{m}\left(N_{C}^{*}\right)\right)$ as defined by Theorem A.12. We have, for $m \geq 2$

$$
\tilde{K}_{m}:=\max \left(K\left(N_{C} \otimes S^{m}\left(N_{C}^{*}\right)\right), K\left(T_{C} \otimes S^{m}\left(N_{C}^{*}\right)\right)\right) .
$$

As in the foliation problem, we consider the sequence of numbers $\left\{\eta_{m}\right\}_{m \geq 1}$ with $\eta_{1}=1$ and, if $m \geq 2$

$$
\eta_{m}:=\tilde{K}_{m} \max _{m_{1}+\cdots+m_{p}+s=m} \eta_{m_{1}} \cdots \eta_{m_{p}}
$$


where, in the maximum, $1 \leq m_{i}<m$ for all $i$ and $s \in \mathbb{N}$. In what follows, $f_{j}^{\bullet}$ (resp. $\phi_{k j}^{\bullet}$ ) stands for either $f_{j}^{h}$ or $f_{j}^{v}$ (resp. $\phi_{k j}^{h}$ or $\phi_{k j}^{v}$ ). As in the previous section, let us expand $\phi_{k j}^{\bullet}\left(h_{j}+f_{j}^{h}, v_{j}+f_{j}^{v}\right)$ appeared in (4.1) and (4.2) in power series of $v_{j}$ and let us define

$$
\begin{aligned}
\phi_{k j}^{\bullet}\left(z_{j}, w_{j}\right) & =: \sum_{Q \in \mathbb{N}_{2}^{d}} \phi_{k j, Q}^{\bullet}\left(z_{j}\right) w_{j}^{Q} \\
\phi_{k j}^{\bullet}\left(h_{j}+f_{j}^{h}\left(h_{j}, v_{j}\right), v_{j}+f_{j}^{v}\left(h_{j}, v_{j}\right)\right) & =: \sum_{Q \in \mathbb{N}_{2}^{d}} h_{k j, Q}^{\bullet}\left(h_{j}\right) v_{j}^{Q}=: h_{k j}^{\bullet}\left(h_{j}, v_{j}\right) .
\end{aligned}
$$

Then we obtain

$$
\sum_{Q \in \mathbb{N}_{2}^{d}} h_{k j, Q}^{\bullet}\left(h_{j}\right) v_{j}^{Q}=\sum_{Q \in \mathbb{N}_{2}^{d}} \phi_{k j, Q}^{\bullet}\left(h_{j}+f_{j}^{h}\left(h_{j}, v_{j}\right)\right)\left(v_{j}+f_{j}^{v}\left(h_{j}, v_{j}\right)\right)^{Q} .
$$

We further expand the first expression on the right-hand side as

$$
\tilde{h}_{k j, Q}^{\bullet}:=\phi_{k j, Q}^{\bullet}\left(h_{j}+f_{j}^{h}\left(h_{j}, v_{j}\right)\right)=\sum_{P \in \mathbb{N}^{n}} \frac{1}{P !} \partial_{h}^{P} \phi_{k j, Q}^{\bullet}\left(h_{j}\right)\left(f_{j}^{h}\left(h_{j}, v_{j}\right)\right)^{P} .
$$

Hence, for any $m \geq 2$,

$$
\left[h_{k j}^{\bullet}\right]^{m}=\sum_{m_{1}+m_{2}=m} \sum_{Q \in \mathbb{N}_{2}^{d}} \sum_{P \in \mathbb{N}^{n}} \frac{1}{P !} \partial_{h}^{P} \phi_{k j, Q}^{\bullet}\left(h_{j}\right)\left[\left(f_{j}^{h}\left(h_{j}, v_{j}\right)\right)^{P}\right]^{m_{1}}\left[\left(v_{j}+f_{j}^{v}\left(h_{j}, v_{j}\right)\right)^{Q}\right]^{m_{2}} .
$$

Let $\left\{f_{j}^{\bullet}\right\}$ be the formal solution of (4.1) and (4.2). Let us first assume that $H^{0}\left(C, T_{C} M \otimes\right.$ $\left.S^{\ell}\left(N_{C}^{*}\right)\right)=0$ for all $\ell \geq 2$. We shall see later on how to get rid of the assumptions. Assume that there is a sequence $\left\{A_{k}\right\}_{k \geq 2}$ of positive numbers such that

$$
\forall k<m \quad\left|\left[f_{j}\right]^{k}\right|_{\hat{U}_{j}} \leq \eta_{k} A_{k}
$$

Let us set

$$
A(t)=\sum_{k \geq 2} A_{k} t^{k}
$$

with $t \in \mathbb{C}$.

Since $\phi_{k j}^{\bullet}$ is holomorphic in $h_{j} \in \hat{U}_{k j}$ and $v_{j}$ in a neighborhood of the origin, we can assume that there is a positive $R$ such that

$$
\sup _{h_{j} \in \hat{U}_{k j}}\left|\phi_{k j, Q}^{\bullet}\left(h_{j}\right)\right| \leq R^{|Q|} .
$$

According to (3.10) and the proof of (3.12), we obtain

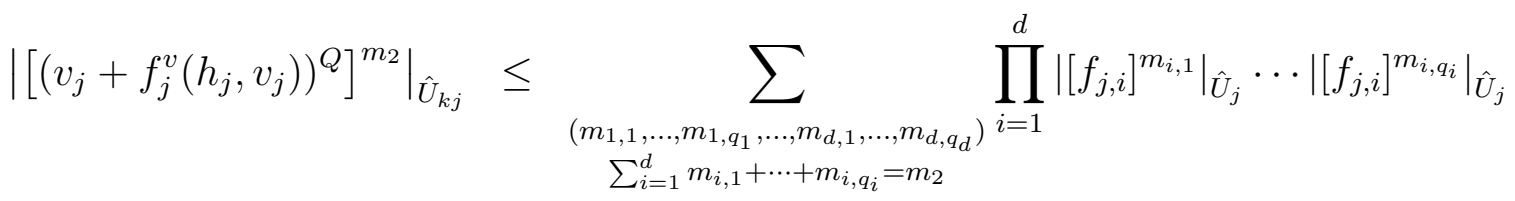

$$
\begin{aligned}
& \leq \sum_{M \in E_{Q, m_{2}}} \prod_{i=1}^{d} \eta_{m_{i, 1}} A_{m_{i, 1}} \cdots \eta_{m_{i, q_{i}}} A_{m_{i, q_{i}}} \\
& \leq \eta_{Q, m_{2}}\left[\left(t+J^{m_{2}-1} A(t)\right)^{|Q|}\right]^{m_{2}} \text {. }
\end{aligned}
$$


On the other hand, let $\mathcal{U}^{*}=\left\{U_{i}^{*}\right\}$ be an open covering of $C$ such that $U_{i}^{*}$ is relatively compact in $U_{i}$. We shall write $\hat{U}_{k}^{*}:=\varphi_{k}\left(U_{k}^{*}\right)$. Let us set

$$
M:=\min _{k} \operatorname{dist}\left(\hat{U}_{k}^{*}, \partial \hat{U}_{k}\right)
$$

Let us consider the index $j$ as fixed and let us estimate the Fischer norm of $\left[\tilde{h}_{k j}^{\bullet}\right]^{m_{1}}$ on $\hat{U}_{k j}^{*}:=\varphi_{j}\left(U_{j} \cap U_{k}^{*}\right)$. We get

$$
\begin{aligned}
\left|\left[\tilde{h}_{k j}^{\bullet}\right]^{m_{1}}\right|_{\hat{U}_{k j}^{*}} & =\sum_{P \in \mathbb{N}^{n}} \frac{1}{P !}\left|\partial_{h}^{P} \phi_{k j, Q}^{\bullet}\left(h_{j}\right)\left[\left(f_{j}^{h}\left(h_{j}, v_{j}\right)\right)^{P}\right]^{m_{1}}\right|_{\hat{U}_{k j}^{*}} \\
& \leq \sum_{P \in \mathbb{N}^{n}}\left(\frac{1}{\operatorname{dist}\left(\hat{U}_{k}^{*}, \partial \hat{U}_{k}\right)}\right)^{|P|}\left|\phi_{k j, Q}^{\bullet}\right|_{\hat{U}_{k j}}\left|\left[\left(f_{j}^{h}\left(h_{j}, v_{j}\right)\right)^{P}\right]^{m_{1}}\right|_{\hat{U}_{k j}^{*}} \\
& \leq \sum_{P \in \mathbb{N}^{n}}\left(\frac{1}{M}\right)^{|P|} R^{|Q|}\left|\left[\left(f_{j}^{h}\left(h_{j}, v_{j}\right)\right)^{P}\right]^{m_{1}}\right|_{\hat{U}_{k j}^{*}} .
\end{aligned}
$$

Since $f_{j}$ is of order $\geq 2$ at $v_{j}=0$, we have $|P| \leq \frac{m_{1}}{2}$ in the above sum. According to estimate (3.10) and following the proof of (3.12), we obtain

$$
\left|\left[\tilde{h}_{k j, Q}^{\bullet}\right]^{m_{1}}\right|_{\hat{U}_{k j}^{*}} \leq \sum_{P \in \mathbb{N}^{n},|P|=0}^{\frac{m_{1}}{2}}\left(\frac{1}{\operatorname{dist}\left(\hat{U}_{k}^{*}, \partial \hat{U}_{k}\right)}\right)^{|P|} R^{|Q|} \eta_{P, m_{1}}\left[A(t)^{|P|}\right]^{m_{1}}
$$

Combining inequalities (4.5) and (4.5), we obtain

$$
\begin{aligned}
& \left|\left[h_{k j}^{\bullet}\right]^{m}\right|_{\hat{U}_{k j}^{*}} \leq \sum_{m_{1}+m_{2}=m} \sum_{Q \in \mathbb{N}_{2}^{d}} \sum_{P \in \mathbb{N}^{n}} \frac{1}{P !}\left|\partial_{h}^{P} \phi_{k j, Q}^{\bullet}\left(h_{j}\right)\left[\left(f_{j}^{h}\left(h_{j}, v_{j}\right)\right)^{P}\right]^{m_{1}}\left[\left(v_{j}+f_{j}^{v}\left(h_{j}, v_{j}\right)\right)^{Q}\right]^{m_{2}}\right|_{\hat{U}_{k j}^{*}} \\
& \leq \sum_{m_{1}+m_{2}=m} \sum_{\substack{Q \in \mathbb{N}^{d} \\
|Q|=2}}^{m_{2}} \sum_{\substack{P \in \mathbb{N}^{n} \\
|P|=0}}^{\frac{m_{1}}{2}}\left(\frac{1}{M}\right)^{|P|} R^{|Q|} \eta_{P, m_{1}}\left[A(t)^{|P|}\right]^{m_{1}} \eta_{Q, m_{2}}\left[\left(t+J^{m_{2}-1} A(t)\right)^{|Q|}\right]^{m_{2}} \\
& \leq \sum_{m_{1}+m_{2}=m} \sum_{\substack{Q \in \mathbb{N}^{d} \\
|Q|=2}}^{m_{2}} \sum_{\substack{P\left|\mathbb{N}^{n}\\
\right| P \mid=0}}^{\frac{m_{1}}{2}}\left[\left(\frac{A(t)}{M}\right)^{|P|}\right]^{m_{1}} \eta_{P, m_{1}} \eta_{Q, m_{2}}\left[\left(R t+R J^{m_{2}-1} A(t)\right)^{|Q|}\right]^{m_{2}} \\
& \leq \tilde{E}_{m}\left[\left(\frac{1}{1-\frac{A(t)}{M}}\right)^{n}\left(\left(\frac{1}{1-(R t+R A(t))}\right)^{d}-1-d(R t+R A(t))\right)\right]^{m} .
\end{aligned}
$$

Here, we have set

$$
\tilde{E}_{m}=\max _{m_{1}+m_{2}=m} \max _{\substack{P \in \mathbb{N}^{n}, Q \in \mathbb{N}^{d} \\|P| \leq \frac{m_{1}}{2}, 2 \leq|Q| \leq m_{2}}} \eta_{P, m_{1}} \eta_{Q, m_{2}}
$$


It remains to estimate the rest of terms in (4.1). We define

$$
\begin{aligned}
B_{m}: & =\left[\varphi_{k j}\left(h_{j}+f_{j}^{h}\left(h_{j}, v_{j}\right)\right)-\varphi_{k j}\left(h_{j}\right)-D \varphi_{k j}\left(h_{j}\right) f_{j}^{h}\left(h_{j}, v_{j}\right)\right]^{m} \\
& =\sum_{l=2}^{\frac{m}{2}} \sum_{|P|=l} \frac{1}{P !} \partial_{h}^{P} \varphi_{k j}\left(h_{j}\right)\left[\left(f_{j}^{h}\right)^{P}\right]^{m} .
\end{aligned}
$$

Hence, as above, we have

$$
\begin{aligned}
\left|B_{m}\right|_{\hat{U}_{k j}^{*}} & \leq\left|\varphi_{k j}\right|_{\hat{U}_{k j}} \sum_{l=2}^{\frac{m}{2}} \sum_{|P|=l}\left(\frac{1}{M}\right)^{|P|}\left[(A(t))^{|P|}\right]^{m} \\
& \leq\left|\varphi_{k j}\right|_{\hat{U}_{k j}}\left[\left(\frac{1}{1-\frac{A(t)}{M}}\right)^{n}-1-n \frac{A(t)}{M}\right]^{m} .
\end{aligned}
$$

By the same reasoning as in the foliation section, the previous estimates on $\hat{U}_{k j}^{*}$ extend to estimates on $\hat{U}_{k j}$, by multiplication by a constant $\tilde{M}$.

Let us define constant $C_{0}:=\max _{k j}\left|\varphi_{k j}\right|_{\hat{U}_{k j}}$ Since we have

$$
\left|\left[f_{j}^{\bullet}\right]^{m}\right|_{\hat{U}_{j}} \leq \tilde{K}_{m} \max _{k}\left|\mathcal{L}_{k j}\left(\left[f_{j}^{\bullet}\right]^{m}\right)\right|_{\hat{U}_{k j}}
$$

then

$$
\begin{aligned}
\left|\left[f_{j}^{\bullet}\right]^{m}\right|_{\hat{U}_{j}} \leq & \tilde{M} \tilde{K}_{m}\left(C_{0}\left[\left(\frac{1}{1-\frac{A(t)}{M}}\right)^{n}-1-n \frac{A(t)}{M}\right]^{m}\right. \\
& \left.+\tilde{E}_{m}\left[\left(\frac{1}{1-\frac{A(t)}{M}}\right)^{n}\left(\left(\frac{1}{1-(R t+R A(t))}\right)^{d}-1-d(R t+R A(t))\right)\right]^{m}\right) .
\end{aligned}
$$

We emphasize that due to the vanishing assumption of the spaces $H^{0}\left(\mathcal{U}, T_{C} M \otimes S^{m}\left(N_{C}^{*}\right)\right)$, $m \geq 2$, the solution of cohomological equation $\mathcal{L}_{k j}\left(\left[f_{j}^{\bullet}\right]^{m}\right)=R^{m}$ is unique and is equal to the minimizing solution obtained in Lemma A.2 and Proposition A.4. Consider the following analytic functional equation :

$$
\begin{aligned}
A(t)= & \tilde{M}\left(C_{0}\left[\left(\frac{1}{1-\frac{A(t)}{M}}\right)^{n}-1-n \frac{A(t)}{M}\right]\right. \\
& \left.+\left(\frac{1}{1-\frac{A(t)}{M}}\right)^{n}\left(\left(\frac{1}{1-(R t+R A(t))}\right)^{d}-1-d(R t+R A(t))\right)\right) .
\end{aligned}
$$

It has a unique analytic solution $A$ of order $\geq 2$ at the origin. Since we have

$$
\tilde{K}_{m} \max \left(1, \tilde{E}_{m}\right) \leq \eta_{m}, \quad\left|\left[f_{j}^{\bullet}\right]^{m}\right|_{\hat{U}_{j}} \leq A_{m} \eta_{m}, \quad m \geq 2
$$

then $\sum_{m \geq 2}\left[f_{j}^{\bullet}\right]^{m}$ converges in a neighborhood of the origin.

Let us see how the general case reduces to the previous one. The issue is that, when considering a solution $\left[f_{j}^{\bullet}\right]^{m}$ of the cohomological equation $\mathcal{L}_{k j}\left(\left[f_{j}^{\bullet}\right]^{m}\right)=R^{m}$, the estimate given by Lemma A.2 and Proposition A.4 might be obtained by another solution. Hence, 
the formal solution might not be the good one for the estimates. So we will need to correct it. As we already emphasized, equations (4.1) and (4.2) read

$$
\mathcal{L}_{k j}\left(\left\{[f]_{i}^{\ell}\right\}\right)=\mathcal{R}_{k j, \ell}\left([f]^{\ell^{\prime}}, \ell^{\prime}<\ell ;[\Phi]^{l}, l \leq \ell\right)
$$

where $\mathcal{R}_{k j, \ell}$ is an analytic function of its arguments. Let us start at $\ell=2$.

(1) $\mathcal{R}_{k j, 2}$ is just a function of the $\left[\Phi_{k j}\right]^{2}$ 's and and we have $\mathcal{L}_{k j}\left([f]^{2}\right)=\mathcal{R}_{k j, 2}$. Let $\left\{\left[\tilde{f}_{j, 2}\right]^{2}\right\}$ be the minimizer solution of this equation obtained by Lemma A.2 and Proposition A.4 and let $\left[k_{j}\right]^{2}:=\left[f_{j}\right]^{2}-\left[\tilde{f}_{j, 2}\right]^{2}$. We have $\left\{\left[k_{j}\right]^{2}\right\} \in H^{0}\left(\mathcal{U}, T_{C} M \otimes\right.$ $\left.S^{2}\left(N_{C}^{*}\right)\right)$

(2) According to lemma Lemma $2.14, F_{j, 2}:=F_{j} \exp \left(-\left[k_{j}\right]^{2}\right)$ linearizes $\Phi_{k j}$ since

$$
F_{j, 2}^{-1} \Phi_{k j} F_{j, 2}=\exp \left(-\left[k_{j}\right]^{2}\right)^{-1} N_{k j} \exp \left(-\left[k_{j}\right]^{2}\right)=N_{k j}
$$

$F_{j, 2}$ is tangent to identity and its 2 nd order term is the minimizer $\left[\tilde{f}_{j}\right]^{2}$.

(3) Assume that $F_{j, \ell}$ linearizes $\Phi_{k j}$, is tangent to identity at the origin and has the minimizers solution up to degree $\ell$ as Taylor expansion at 0 . This means that $F_{j, \ell}=I d+\sum_{l=2}^{\ell}\left[\tilde{f}_{j, l}\right]^{l}+\sum_{l \geq \ell+1}\left[f_{j, \ell}\right]^{l}$. Let us write the conjugacy equation. By induction we have, for all $2 \leq l \leq \ell$,

$$
\mathcal{L}_{k j}\left(\left\{\left[\tilde{f}_{i, l}\right]^{l}\right\}\right)=\mathcal{R}_{k j, l}\left(\left\{\left[\tilde{f}_{i, l^{\prime}}\right]^{l^{\prime}}\right\}_{i}, l^{\prime}<l ;[\Phi]^{m}, m \leq l\right) .
$$

Furthermore, it satisfies at degree $\ell+1$

$$
\mathcal{L}_{k j}\left(\left\{\left[f_{i, \ell+1}\right]^{\ell+1}\right\}\right)=\mathcal{R}_{k j, \ell+1}\left(\left\{\left[\tilde{f}_{i, \ell^{\prime}}\right]^{\ell^{\prime}}\right\}_{i}, \ell^{\prime} \leq \ell ;[\Phi]^{m}, m \leq \ell+1\right) .
$$

Let $\left[\tilde{f}_{i, \ell+1}\right]^{\ell+1}$ be the minimizer solution of the above cohomological equation. Let $\left[k_{i, \ell+1}\right]^{\ell+1}=\left[f_{i, \ell+1}\right]^{\ell+1}-\left[\tilde{f}_{i, \ell+1}\right]^{\ell+1}$. As above, it defines an element of $H^{0}\left(\mathcal{U}, T_{C} M \otimes\right.$ $\left.S^{\ell+1}\left(N_{C}^{*}\right)\right)$. Let us set $F_{j, \ell+1}=F_{j, \ell} \exp \left(\left[k_{j, \ell+1}\right]^{\ell+1}\right)^{-1}$. Then it linearizes $\Phi_{k j}$ and has the minimizers solution up to degree $\ell+1$ as Taylor expansion at $0: F_{j, \ell+1}=$ $I d+\sum_{l=2}^{\ell+1}\left[\tilde{f}_{j, l}\right]^{l}+\sum_{l \geq \ell+2}\left[f_{j, \ell+1}\right]^{l}$.

(4) Since $F_{j, \ell+1} F_{j, \ell}^{-1}=I+O(\ell+1)$, the sequence $\left\{F_{j, \ell}\right\}_{\ell}$ converges in the space of formal power series to $\tilde{F}_{j}$. Furthermore, $\left\{\tilde{F}_{j}\right\}$ linearizes $\left\{\Phi_{k j}\right\}$ as each $\left\{F_{j, \ell}\right\}_{j}$ does. The Taylor expansion of $\tilde{F}_{j}$ at the origin is

$$
\tilde{F}_{j}=I d+\sum_{l \geq 2}\left[\tilde{f}_{j, l}\right]^{l} .
$$

(5) We can estimate the $\left[\tilde{f}_{j, l}\right]^{l}$ as we did above in the case of vanishing cohomology since the Taylor coefficient are minimizer solutions of the same equations.

Hence, we are done.

In summary, we have proved the following theorem.

Theorem 4.1. Let $C$ be an embedded compact manifold in $M$. Assume that the embedding is linearizable by a formal holomorphic mapping which is tangent to the identity and preserves the splitting of $T_{C} M$, and $N_{C}$ is unitary. Suppose that $\left\{\eta_{m}\right\}_{m \geq 1}$ defined by (4.3) satisfy $\eta_{m} \leq L_{0} L^{m}$, for some positive numbers $L_{0}, L$ and for all $m$. Then the embedding is actually holomorphically linearizable. 
We remark that in general there is a rigid theory on deformations in an analytic family of complex complex manifolds due to Kodaira [24]. Strengthening Corollary 3.3, we finish the section with the following corollary. This may be regarded as a rigidity for a simplify connected manifold.

Corollary 4.2. Keep the assumptions in Theorem 4.1. Assume further that $C$ is simply connected. Then a neighborhood of $C$ in $M$ is biholomorphic to $C \times B^{d}$ where $B^{d}$ is the unit ball in $\mathbf{C}^{d}$.

Proof. We already know that $M$ admits a horizontal foliation by Corollary 3.3. To show that each leaf is biholomorphic to $C$, we may assume that $M=N_{C}$ and we will use the projection $\pi: N_{C} \rightarrow C$. We fix $x_{0} \in C$. We take a point $p \in \pi^{-1}\left(x_{0}\right)$ close to $C$. Let $L$ be the (connected) leaf of the foliation containing $p$. Then $L$ intersects each fiber of $N_{C}$ at a unique point. To verify this, we connect a point in $x \in C$ to $x_{0}$ by a continuous path $\gamma$ in $C$ with $\gamma(0)=x_{0}$ and $\gamma(1)=x$. By continuation along leaves, we can find a lifted continuous path $\tilde{\gamma}$ and the germ $L_{\gamma}^{*}(t)$ at $\tilde{\gamma}(t)$ of a leaf $L_{\gamma}(t)$ such that $\pi(\tilde{\gamma}(t))=\gamma(t)$. Note that $L_{\gamma}^{*}\left(t^{\prime}\right), L_{\gamma}^{*}(t)$ are contained in the same leaf on which $\pi$ is injective, when $t^{\prime}$ is sufficiently close to $t$. The lifting $\tilde{\gamma}(1)$ is independent of $\gamma$. Indeed if $\gamma^{\theta}(a \leq \theta \leq b)$ is a continuous family of paths connecting $x_{0}$ to $x$. Let $L_{\gamma^{\theta}}$ be the leaf associated to $\gamma^{\theta}$. Then $\widetilde{\gamma^{\theta}}(t) \in L_{\gamma^{\theta_{0}}}(t)$ when $\theta$ is sufficiently close to $\theta_{0}$, as $L_{\gamma^{\theta}}(0)=L_{\gamma^{a}}(0)$ as a leaf near $p$.

Obviously, $x \mapsto \tilde{\gamma}(1)$ gives a biholomorphism from $C$ onto the leaf through $p$. And $(x, v) \rightarrow \tilde{\gamma}(1)$ defines a biholomorphisms from $C \times B$ into $N_{C}$, where $B$ is a small neighborhood of $0 \in \pi^{-1}\left(x_{0}\right)$.

\section{The FUlL LinearizATION}

The main purpose of this section is to solve the linearization problem in the general setting (i.e. $N_{C}$ not necessarily being flat) under a general hypotheses on the existence of bounds to the cohomology equations. At the end of the section we will illustrate the results with Arnold's examples [2], following computations by Arnol'd [3].

We shall devise a Newton scheme to solve the linearization of the neighborhood problem. Let us recall the condition.

$\left(L_{m}\right)$ : The neighborhood of $C$ agrees with the neighborhood of the zero section of the normal bundle up to order $m$.

That embedding of $C$ has property $\left(L_{m}\right)$ means that the order of $\left(\phi_{k j}^{h}\left(h_{j}, v_{j}\right), \phi_{k j}^{v}\left(h_{j}, v_{j}\right)\right)$ along $v_{j}=0$ as defined in (5.16) is $\geq m+1$.

Assuming that $\left(L_{m}\right)$ holds. We shall assume either that $H^{0}\left(C, T C \otimes S^{p} N_{C}^{*}\right)=0,2 \leq p \leq$ $2 m$ or that $N_{C}$ is flat. According to Lemma 2.16 (c) and (d), the following linearization step in the Newton method is fulfilled:

$\left(N_{m}\right)$ : If $\left\{\Phi_{k j}\right\} \in L_{m}$, then $\left\{F_{k}^{-1} \Phi_{k j} F_{j}\right\} \in L_{2 m}$ for some $\left\{F_{j}=I+f_{j}\right\}$ with $f_{j}\left(h_{j}, v_{j}\right)=$ $O\left(\left|v_{j}\right|^{m+1}\right)$. 
5.1. Domains for iteration and the Donin condition. Following Lemma A.6 and Proposition A.19, we shall consider a family of nested coverings $\mathcal{U}^{r}=\left\{U_{i}^{r}\right\}_{i \in I}$ of $C$ with $r_{*} \leq r \leq r^{*}$. Let us fix a trivialization of $N_{C}^{*}$ (resp. TC) over $U_{i}^{r^{*}}$ by fixing a holomorphic basis $e_{i}=\left(e_{i, 1}, \ldots, e_{i, n+d}\right)$ of $T_{C} M$ on $U_{i}^{r^{*}}$.

We first define various domains. Let $\hat{U}_{j}^{r}:=\varphi_{j}\left(U_{j}^{r}\right)=\Delta_{n}^{r}$ and $U_{k j}^{r}:=U_{k}^{r} \cap U_{j}^{r}$. We have $U_{k j}^{r}=U_{j k}^{r}$. Define $\hat{U}_{k j}^{r}=\varphi_{j}\left(U_{k j}^{r}\right)$. Then

$$
\varphi_{k j}\left(U_{k j}^{r}\right)=\hat{U}_{j k}^{r}
$$

Donin Condition. Let $\mathcal{U}^{r}$ be a family of nested covering of $C$ for $r_{*}<r<r^{*}$. Let $E^{\prime}=T C$ or $N_{C}$. Suppose that there are constants $D\left(E^{\prime} \otimes S^{m} N_{C}^{*}\right)$ for $m=2,3, \ldots$ such that for all $r^{\prime}, r^{\prime \prime}$ with $r_{*}<r^{\prime \prime}<r^{\prime}<r<r^{*}$ and $r^{\prime}-r^{\prime \prime} \leq r^{*}-r$, and all $f \in Z^{1}\left(\mathcal{U}^{r^{\prime}}, E^{\prime} \otimes S^{m} N_{C}^{*}\right)$ with $f=0$ in $H^{1}\left(\mathcal{U}^{r^{\prime}}, E^{\prime} \otimes S^{m} N_{C}^{*}\right)$, there is a solution $u \in C^{0}\left(\mathcal{U}^{r^{\prime \prime}}, E^{\prime} \otimes S^{m} N_{C}^{*}\right)$ to $\delta u=f$ such that

$$
\max _{j} \sup \left|u_{j}\right|_{L^{\infty}\left(\hat{U}_{j}^{r^{\prime \prime}}\right)} \leq \frac{D\left(E^{\prime} \otimes S^{m} N_{C}^{*}\right)}{\left(r^{\prime}-r^{\prime \prime}\right)^{\tau}} \max _{k, j}\left|f_{k j}\right|_{L^{\infty}\left(\hat{U}_{k j}^{r^{\prime}}\right)},
$$

where $D\left(E^{\prime} \otimes S^{m} N_{C}^{*}\right)$ is independent of $r^{\prime}, r^{\prime \prime}$ and $f$ and $\tau=\tau\left(N_{C}^{*}\right)$ is independent of $m$.

In what follow, we shall express sections of bundles in coordinates. It is more convenient to express domains by using the trivialization of the vector bundle $N_{C}$. Recall that the $N_{C}$ has trivializations $N_{j}$ and transition functions $N_{k j}$. Let $B_{d}^{r}$ be the ball of radius $r$ in $\mathbf{C}^{d}$ centered at the origin. Thus, we define

$$
\begin{gathered}
\hat{V}_{j}^{r}=N_{j}\left(V_{j}^{r}\right)=\hat{U}_{j}^{r} \times B_{d}^{r}, \quad V_{i_{0} \cdots i_{q}}^{r}:=V_{i_{0}}^{r} \cap \cdots \cap V_{i_{q}}^{r}, \\
\hat{V}_{i_{0} \cdots i_{q}}^{r}:=N_{i_{q}}\left(V_{i_{0} \cdots i_{q}}^{r}\right) \subset \varphi_{i_{q}}\left(U_{i_{0} \cdots i_{q}}^{r}\right) \times \mathbf{C}^{d}, \\
\hat{V}_{j k}^{r}=N_{k j}\left(\hat{V}_{k j}^{r}\right), \quad N_{k j}=N_{j k}^{-1} \quad \text { on } \hat{V}_{k j}^{r}, \\
N_{k i} N_{i j}=N_{k j} \quad \text { on } \hat{V}_{k i j}^{r} .
\end{gathered}
$$

Denote the corresponding domains by $\tilde{V}_{j}^{r}, \tilde{V}_{k j}^{r}$ when $N_{j}$ are replaced by $\Phi_{j}$. Then we still have the above relations when $N_{j}, N_{k j}$ are replaced by $\Phi_{j}, \Phi_{k j}$. We know that $\Phi_{k j}$ are perturbations of the transition functions $N_{k j}$ of the normal bundle of $C$ in $M$, which are defined on different domains but in the same space. We will however work on domains $\hat{V}_{k j}^{r}$ for $\Phi_{k j}$, instead of $\tilde{V}_{k j}^{r}$.

With notation of section 2.6, for $L \geq 1$ and for $r_{*} \leq r \leq r^{*}$, we consider a cochain $\left\{f_{I}\right\} \in C^{q+1}\left(\mathcal{U}^{r}, \mathcal{O}\left(T_{C} M \otimes S^{L}\left(N_{C}^{*}\right)\right)\right)$, given by

$$
f_{I}:=f_{i_{0} \cdots i_{q}}(p)=\sum_{\lambda=1}^{n+d} \sum_{|Q|=L} f_{i_{0} \cdots i_{q} ; Q}^{\lambda}\left(z_{i_{q}}(p)\right) e_{i_{0}, \lambda}(p) \otimes\left(w_{i_{q}}^{*}(p)\right)^{Q}
$$

where $I=\left(i_{0}, \ldots, i_{q}\right) \in \mathcal{I}^{q+1}$. Recall that $\hat{V}_{I}^{r}=N_{i_{q}}\left(V_{i_{0}}^{r} \cap \cdots \cap V_{i_{q}}^{r}\right)$. Define

$$
\left|f_{I}\right|_{r}=\sup _{\left(h_{i_{q}}, v_{i_{q}}\right) \in \hat{V}_{I}^{r}}\left|\sum_{Q} f_{I, Q}\left(h_{i_{q}}\right) v_{i_{q}}^{Q}\right| .
$$

We also set $\left|\left\{f_{I}\right\}\right|_{r}=\max _{I}\left|f_{I}\right|_{r}$. 
Note that $\hat{V}_{j}^{r}=\hat{U}_{j} \times B_{d}^{r}$ are product domains. Also,

$$
\hat{U}_{k j}^{r} \times B_{d}^{c_{*} r} \subset \hat{V}_{k j}^{r} \subset \hat{U}_{k j}^{r} \times B_{d}^{c^{*} r}, \quad c_{*} \leq 1 \leq c^{*} .
$$

Define $B_{k j}^{r}\left(h_{j}\right)$ to be $\left\{v_{j} \in B_{d}^{r}: t_{k j}\left(h_{j}\right) v_{j} \in B_{d}^{r}\right\}$. The skewed domain $\hat{V}_{k j}^{r}$ can be described as follows:

$$
\left(h_{j}, v_{j}\right) \in \hat{V}_{k j}^{r} \quad \text { if and only if } \quad h_{j} \in \hat{U}_{k j}^{r}, v_{j} \in B_{k j}^{r}\left(h_{j}\right) .
$$

Next, we note that the $d$-torus action $\left(h_{j}, v_{j}\right) \rightarrow\left(h_{j},\left(\zeta_{1} v_{1}, \ldots, \zeta_{d} v_{d}\right)\right)$ with $\zeta \in\left(S^{1}\right)^{d}$ does not preserve $\hat{V}_{k j}^{r}$ when $t_{k j}\left(h_{j}\right)$ is not diagonal. Nevertheless, the $\hat{V}_{k j}^{r}$ has a disc structure:

$$
\left(h_{j}, \zeta v_{j}\right) \in \hat{V}_{k j}^{r}, \quad \forall\left(h_{j}, v_{j}\right) \in \hat{V}_{k j}^{r}, \quad \forall \zeta \in \Delta .
$$

Indeed, suppose that $\left(h_{j}, v_{j}\right) \in \hat{V}_{k j}^{r}$. Then $h_{j} \in \hat{U}_{k j}^{r}$ and $\left(h_{j}, v_{j}\right)=N_{j}(p)$ with $p \in V_{k}^{r} \cap V_{j}^{r}$ and $N_{k}(p)=\left(h_{k}, v_{k}\right) \in \hat{V}_{k}^{r}$. By definition, $\hat{V}_{j}^{r}=\hat{U}_{j} \times B_{d}^{r}$. Take $\tilde{p}=N_{j}^{-1}\left(h_{j}, \zeta v_{j}\right)$. We have $\tilde{p} \in V_{j}^{r}$ and $N_{k}(\tilde{p})=\left(h_{k}, t_{k j}\left(\zeta v_{j}\right)\right)=\left(h_{k}, \zeta t_{k j}\left(v_{j}\right)\right) \in \hat{U}_{k j}^{r} \times B_{d}^{r}$.

Throughout this section, we use

$$
\left|u_{j}\right|_{\rho}=\sup _{\left(h_{j}, v_{j}\right) \in \hat{V}_{j}^{\rho}}\left|u_{j}\left(h_{j}, v_{j}\right)\right|, \quad\left|u_{k j}\right|_{\rho}=\sup _{\left(h_{j}, v_{j}\right) \in \hat{V}_{k j}^{\rho}}\left|u_{k j}\left(h_{j}, v_{j}\right)\right|
$$

where $u_{j}, u_{k j}$ are functions on $\hat{V}_{j}^{r}$ and $\hat{V}_{k j}^{r}$, respectively. We also define $\left|\left\{u_{I}\right\}\right|_{\rho}=\max _{I}\left|u_{I}\right|_{\rho}$.

We now prove the following.

Lemma 5.1. Let $u_{k j}$ be a holomorphic function on $\hat{V}_{k j}^{r}$ with $r_{*}<r<\tilde{r}<r^{*}$. Suppose that

$$
\hat{V}_{k j}^{r_{*}} \neq \emptyset .
$$

For $0<\theta<1$ with $\theta r>r^{*}$, we have

$$
\begin{gathered}
\left|u_{k j}\right|_{\theta r} \leq \theta^{m}\left|u_{k j}\right|_{r}, \quad \text { if } u_{k j}\left(h_{j}, v_{j}\right)=O\left(\left|v_{j}\right|^{m}\right) ; \quad\left|\left[u_{k j}\right]^{\ell}\right|_{r} \leq\left|u_{k j}\right|_{r} ; \\
\sum_{\ell=i}^{\infty}\left|\left[u_{k j}\right]^{\ell}\right|_{\theta r} \leq \frac{\theta^{i}}{1-\theta}\left|u_{k j}\right|_{r} .
\end{gathered}
$$

Proof. Let $u=u_{k j}$. The first inequality follows from the Schwarz lemma applied to the holomorphic function $\zeta \rightarrow u\left(h_{j}, \zeta v_{j}\right)$ on the unit disk for fixed $\left(h_{j}, v_{j}\right) \in \hat{V}_{k j}^{r}$. Note that $[u]^{i}\left(h_{j}, \zeta v_{j}\right)=\zeta^{i}[u]^{i}\left(h_{j}, v_{j}\right)$. Thus the second inequality follows directly by averaging,

$$
[u]^{\ell}\left(h_{j}, v_{j}\right)=\frac{1}{2 \pi i} \int_{\zeta \in \partial \Delta} u\left(h_{j}, \zeta v_{j}\right) \frac{d \zeta}{\zeta^{\ell+1}}, \quad\left(h_{j}, v_{j}\right) \in \hat{V}_{k j}^{r} .
$$

The last inequality follows from the first two inequalities.

For the rest of this section, we rename $r$ in the Donin Condition by $\tilde{r}$ which is fixed now. We will let $r$ vary in $\left(r_{*}, \tilde{r}\right)$.

Lemma 5.2. Let $r_{*}<\theta r<r<\tilde{r}<r^{*}<1$. Fix $k, j \in \mathcal{I}$. Suppose that $\left(1-\theta^{4}\right) r<r^{*}-\tilde{r}$ and (5.5) holds. 
(a) We have

$$
\operatorname{dist}\left(\hat{V}_{j}^{\theta r}, \partial \hat{V}_{j}^{r}\right) \geq r(1-\theta) / C_{0}, \quad \operatorname{dist}\left(\hat{V}_{k j}^{\theta r}, \partial \hat{V}_{k j}^{r}\right) \geq r(1-\theta) / C_{0},
$$

for some constant $C_{0}$.

(b) Assume further that $\theta^{4} r>r_{*}$. There exists a constant $C_{0}^{*}$ such that if $F_{j}=I+f_{j}$ satisfy

$$
\left|f_{j}\right|_{\theta^{2} r} \leq(1-\theta) r / C_{0}^{*}
$$

then we have

$$
\begin{aligned}
& F_{j}\left(\hat{V}_{j}^{\theta^{2} r}\right) \subset \hat{V}_{j}^{\theta r}, F_{j}\left(\hat{V}_{k j}^{\theta^{2} r}\right) \subset \hat{V}_{k j}^{\theta r}, \\
& F_{j}^{-1}\left(\hat{V}_{j}^{\theta^{4} r}\right) \subset \hat{V}_{j}^{\theta^{3} r}, \quad F_{j} F_{j}^{-1}=I \text { on } \hat{V}_{j}^{\theta^{4} r} .
\end{aligned}
$$

Proof. (a) The $\hat{V}_{j}^{r}$ is the product domain $\hat{U}_{j}^{r} \times B_{d}^{r}$. Thus the first inequality in (5.6) holds trivially since $\hat{U}_{j}^{r}$ is a polydisc. Note that $\hat{V}_{k j}^{r}$ are open sets. Then $\delta:=\operatorname{dist}((h, v),(\tilde{h}, \tilde{v}))=$ $\operatorname{dist}\left(\hat{V}_{k j}^{\theta r}, \partial \hat{V}_{k j}^{r}\right)$ is attained by

$$
(h, v) \in \partial \hat{V}_{k j}^{\theta r}, \quad(\tilde{h}, \tilde{v}) \in \partial \hat{V}_{k j}^{r} .
$$

If $\tilde{h} \in \partial \hat{U}_{k j}^{r}$, we immediately get $\delta \geq \operatorname{dist}\left(\hat{U}_{k j}^{\theta r}, \partial \hat{U}_{k j}^{r}\right) \geq(1-\theta) r / C$ by Lemma A.6. Assume that $\tilde{h} \in \hat{U}_{k j}^{r}$. Then by the continuity of the function $t_{k j}, \tilde{v}$ must be in $\partial B_{k j}^{r}(\tilde{h})$. Otherwise, both $\tilde{h} \in \hat{U}_{k j}^{r}$ and $\tilde{v} \in B_{k j}^{r}(\tilde{h})$ are interior points of the two sets, then any small perturbation of $(\tilde{h}, \tilde{v})$ still satisfies the second condition in (5.10). The last assertion implies that $(\tilde{h}, \tilde{v})$ cannot be a boundary point and we get a contradiction. Therefore, we have

$$
\tilde{v} \in \partial B_{d}^{r} \quad \text { or } \quad t_{k j}(\tilde{h}) \tilde{v} \in \partial B_{d}^{r} .
$$

The first case yields $|\tilde{v}-v| \geq \operatorname{dist}\left(B_{d}^{\theta r}, \partial B_{d}^{r}\right)=(1-\theta) r$. We now consider the second case. By assumption $t_{k j}$ is holomorphic in $\bar{\omega}$ for a neighborhood $\omega$ of $\hat{U}_{k j}$. Thus there is $\delta_{*}>0$ depending only on $\hat{U}_{k j}$ such that if $h \in \hat{U}_{k j}$ and $|\tilde{h}-h|<\delta_{*}$, then the line segment $\gamma$ connecting $h, \tilde{h}$ is contained in $\omega$. Suppose that $|\tilde{h}-h|<(1-\theta) r / C_{1}$ for $C_{1}$ to be determined so that $(1-\theta) r / C_{1}<\delta_{*}$. Applying the mean-value-theorem to $t_{k j}(\gamma)$ and using $t_{k j}(h) v \in B_{d}^{\theta r}$, we get

$$
\begin{aligned}
C_{4}|\tilde{v}-v| & \left.\geq\left|t_{k j}(\tilde{h})(\tilde{v}-v)\right| \geq|| t_{k j}(\tilde{h}) \tilde{v}-t_{k j}(h) v\right)|-|\left(t_{k j}(\tilde{h})-t_{k j}(h)\right) v|| \\
& \geq(1-\theta) r-C_{5}|\tilde{h}-h||v| \geq(1-\theta) r / 2,
\end{aligned}
$$

when $C_{1}$ is sufficiently large. Thus we get $\operatorname{dist}\left(\hat{U}_{k j}^{\theta r}, \partial \hat{U}_{k j}^{r}\right) \geq(1-\theta) r / C$ as in the first case. If $|\tilde{h}-h| \geq(1-\theta) r / C_{1}$, the required estimate is immediate.

(b) Note that $\theta>r_{*}$. By choosing a larger $C_{0}^{*}$, (5.8) follows from (5.6) immediately. We want to find $F^{-1}$. By (5.7) and the Cauchy estimate, we know that

$$
\left|\partial_{h_{j}} f_{j}\left(h_{j}, v_{j}\right)\right|+\left|\partial_{v_{j}} f_{j}\left(h_{j}, v_{j}\right)\right| \leq C_{6} / C_{0}^{*}, \quad \forall\left(h_{j}, v_{j}\right) \in \hat{V}_{j}^{\theta^{3} r} .
$$

Note that $\overline{V_{j}^{r}}=\overline{\hat{U}_{j}^{r}} \times \overline{B_{d}^{r}}$ is convex. By (5.11) and the fundamental theorem of calculus, we have

$$
\left|f_{j}\left(p_{1}\right)-f_{j}\left(p_{0}\right)\right| \leq C_{7}\left|p_{1}-p_{0}\right| / C_{0}^{*}, \quad \forall p_{0}, p_{1} \in \hat{V}_{j}^{\theta^{3} r}
$$


Suppose that $C_{0}^{*}>2 C_{7}$. Then $F_{j}: \hat{V}_{j}^{\theta^{3} r} \rightarrow \hat{V}_{j}^{\theta^{2} r}$ is injective, and $T\left(h_{j}, v_{j}\right)=\left(\tilde{h}_{j}, \tilde{v}_{j}\right)-$ $f_{j}\left(h_{j}, v_{j}\right)$ defines a contraction mapping on $\hat{V}_{j}^{\theta^{3} r}$, if $\left(\tilde{h}_{j}, \tilde{v}_{j}\right) \in \hat{V}_{j}^{\theta^{4} r}$ and $C_{0}^{*}$ is sufficiently large. This gives us (5.9).

In this section, we change notation and let

$$
f_{j}^{\bullet}=\left(f_{j}^{h}, f_{j}^{v}\right), \quad \phi_{k j}^{\bullet}=\left(\phi_{k j}^{h}, \phi_{k j}^{v}\right) .
$$

Lemma 5.3. Let $r_{*}<\theta r<r<\tilde{r}<r^{*}<1$. Suppose that $\hat{V}_{k j}$ satisfies (5.5). There exists a constant $C_{1}^{*}$ such that if

$$
\left|\phi_{k j}^{\bullet}\right|_{r} \leq(1-\theta) r / C_{1}^{*}
$$

then we have

$$
\Phi_{k j}\left(\hat{V}_{k j}^{\theta r}\right) \subset \hat{V}_{j k}^{r}
$$

Proof. Note that $\theta>r_{*}$. Since $\Phi_{k j}-N_{k j}=\phi_{k j}^{\bullet}$ and $N_{k j}\left(\hat{V}_{k j}^{\theta r}\right)=\hat{V}_{j k}^{\theta r}$, the assertion follows from (5.6) and (5.12) for sufficiently large $C_{1}^{*}$.

Proposition 5.4. Let $r_{*}<\theta^{7} r<r<\tilde{r}<r^{*}<1$. Assume that $\hat{V}_{k j}$ satisfies (5.5). Suppose that $\Phi_{k j}=N_{k j}+\phi_{k j}^{\bullet}$ satisfy (5.12). Let $F_{j}=I+f_{j}$ satisfy $f_{j}\left(h_{j}, v_{j}\right)=O\left(\left|v_{j}\right|^{2}\right)$.

Suppose $\tilde{\Phi}_{k j}=F_{k}^{-1} \Phi_{k j} F_{j}=N_{k j}+\tilde{\phi}_{k j}^{\bullet}$. There exists a constant $C_{2}^{*}$ such that if

$$
\left|\left\{f_{j}\right\}\right|_{\theta^{2} r} \leq(1-\theta) r / C_{2}^{*}
$$

and $\tilde{\phi}_{k j}^{\bullet}\left(h_{j}, v_{j}\right)=O\left(\left|v_{j}\right|^{\widetilde{m}}\right)$, then

$$
\begin{gathered}
\left|\left\{\tilde{\phi}_{k j}^{\bullet}\right\}\right|_{\theta^{7} r} \leq C_{2} \theta^{\widetilde{m}}\left(\left|\left\{f_{j}\right\}\right|_{\theta^{2} r}+\left|\left\{\phi_{k j}^{\bullet}\right\}\right|_{r},\right. \\
\left|\left\{\tilde{\phi}_{k j}^{\bullet}\right\}\right|_{\theta^{7} r} \leq C_{2} \theta^{\tilde{m}}(1-\theta) r .
\end{gathered}
$$

Proof. Let us write $\tilde{\Phi}_{k j}=N_{k j}+\tilde{\phi}_{k j}^{\bullet}$ and $F_{k}^{-1}=I+g_{k}$. Thus

$$
\begin{aligned}
\tilde{\phi}_{k j}^{h}= & g_{k}^{h} \circ \Phi_{k j} \circ F_{j}+\phi_{k j}^{h} \circ F_{j}+\left(\varphi_{k j}\left(I+f_{j}^{h}\right)-\varphi_{k j}\right), \\
\tilde{\phi}_{k j}^{v}= & g_{k}^{v} \circ \Phi_{k j} \circ F_{j}+\phi_{k j}^{v} \circ F_{j} \\
& +\left(t_{k j}\left(h_{j}+f_{j}^{h}\right)-t_{k j}\left(h_{j}\right)\right) \times\left(v_{j}+f_{j}^{v}\right)+t_{k j}\left(h_{j}\right) \times f_{j}^{v}\left(h_{j}, v_{j}\right) .
\end{aligned}
$$

According to $(5.9)$, we have $F_{k}\left(I+g_{k}\right)=I$ on $\hat{V}_{k}^{\theta^{4} r}$. Thus $g_{k}=-f_{k} \circ F_{k}^{-1}$ implies that

$$
\left|g_{k}\right|_{\theta^{4} r} \leq\left|f_{k}\right|_{\theta^{3} r}
$$

For $\left(h_{j}, v_{j}\right) \in \hat{V}_{k j}^{\theta^{6} r}$, using $\operatorname{dist}\left(\hat{U}_{k j}^{\theta^{6} r}, \partial \hat{U}_{k j}^{\theta^{5} r}\right) \geq(1-\theta) \theta^{5} r / C_{0}$, we can obtain $\mid t_{k j}\left(h_{j}+\right.$ $\left.f_{j}^{h}\left(h_{j}, v_{j}\right)\right)-t_{k j}\left(h_{j}\right)\left|\leq C_{3}\right| f^{h}\left(h_{j}, v_{j}\right) \mid$ and $\left|\varphi_{k j}\left(h_{j}+f_{j}^{h}\left(h_{j}, v_{j}\right)\right)-\varphi_{k j}\left(h_{j}, v_{j}\right)\right| \leq C_{3}\left|f_{j}\left(h_{j}, v_{j}\right)\right|$. Nesting domains and using (5.12), (5.13) and hence (5.7), we obtain by Lemma 5.2 in which $r$ is replaced by $\theta^{5} r$ :

$$
\begin{gathered}
\left|\left\{\tilde{\phi}_{k j}^{\bullet}\right\}\right|_{\theta^{6} r} \leq C_{4}\left(\left|\left\{f_{j}\right\}\right|_{\theta r}+\left|\left\{\phi_{k j}^{\bullet}\right\}\right|_{r},\right. \\
\left|\left\{\tilde{\phi}_{k j}^{\bullet}\right\}\right|_{\theta^{6} r} \leq C_{4}(1-\theta) r .
\end{gathered}
$$

Applying Schwarz inequality, we get (5.14)-(5.15). 

by

When we apply the above to iteration, the new $\Phi_{k j}$ in the sequence of iteration is defined

$$
\left(F_{k}^{(m)}\right)^{-1}\left(\cdots\left(\left(F_{k}^{(1)}\right)^{-1} \Phi_{k j} F_{j}^{(1)}\right) \cdots\right) F_{j}^{(m)}
$$

on $\hat{V}_{k j}^{r_{m+1}}$ with $F_{j}^{(m)}\left(\hat{V}_{k j}^{r_{m+1}}\right) \subset \hat{V}_{k j}^{r_{m}}$.

Let us find $\left[f_{j}\right]_{m+1}^{2 m}\left(h_{j}, v_{j}\right)$, a polynomial of order $\geq m+1$ and of degree $\leq 2 m$ in $v_{j}$ (holomorphic in $h_{j}$ ), such that $\left\{F_{k}^{-1} \Phi_{k j} F_{j}\right\} \in L_{2 m}$ holds for some $\left\{F_{j}=I+\left[f_{j}\right]_{m+1}^{2 m}\right\}$.

Let us consider the neighborhood written in the new coordinates $\left\{F_{j}\right\}$. We obtain for $\left(h_{k}, v_{k}\right)=\hat{\Phi}_{k j}\left(h_{j}, v_{j}\right)$ :

$$
\begin{aligned}
& h_{k}=\hat{\Phi}_{k j}^{h}\left(h_{j}, v_{j}\right):=\varphi_{k j}\left(h_{j}\right)+\hat{\phi}_{k j}^{h}\left(h_{j}, v_{j}\right), \\
& v_{k}=\hat{\Phi}_{k j}^{v}\left(h_{j}, v_{j}\right):=t_{k j}\left(h_{j}\right) v_{j}+\hat{\phi}_{k j}^{v}\left(h_{j}, v_{j}\right) .
\end{aligned}
$$

We assume that $\hat{\phi}_{k j}^{\bullet}:=\left(\hat{\phi}_{k j}^{h}, \hat{\phi}_{k j}^{v}\right)$ has order $\geq 2 m+1$ at $v_{j}=0$.

Let us write down the horizontal and vertical equations for the linearization problem: $F_{k} \hat{\Phi}_{k j}=\Phi_{k j} F_{j}$. We obtain the horizontal equation

$$
\begin{aligned}
\varphi_{k j}\left(h_{j}\right) & +\hat{\phi}_{k j}^{h}\left(h_{j}, v_{j}\right)+f_{k}^{h}\left(\varphi_{k j}+\hat{\phi}_{k j}^{h}, t_{k j}\left(h_{j}\right) v_{j}+\hat{\phi}_{k j}^{v}\right) \\
& =\varphi_{k j}\left(h_{j}+f_{j}^{h}\left(h_{j}, v_{j}\right)\right)+\phi_{k j}^{h}\left(h_{j}+f_{j}^{h}, v_{j}+f_{j}^{v}\right) .
\end{aligned}
$$

The vertical equation reads

$$
\begin{aligned}
t_{k j}\left(h_{j}\right) v_{j} & +\hat{\phi}_{k j}^{v}\left(h_{j}, v_{j}\right)+f_{k}^{v}\left(\varphi_{k j}+\hat{\phi}_{k j}^{h}, t_{k j}\left(h_{j}\right) v_{j}+\hat{\phi}_{k j}^{v}\right) \\
& =t_{k j}\left(h_{j}+f_{j}^{h}\right)\left(v_{j}+f_{j}^{v}\right)+\phi_{k j}^{v}\left(h_{j}+f_{j}^{h}, v_{j}+f_{j}^{v}\right) .
\end{aligned}
$$

We will interpret the above identity as power series in $v_{j}$ with coefficients being holomorphic in $\varphi_{j}\left(U_{k} \cap U_{j}\right)$. In what follows, degrees or orders of sections are considered w.r.t. $v_{j}$ at $v_{j}=0$.

5.2. A Newton method for the full linearization. For this problem, the two previous equations can be written as

$$
\mathcal{L}_{k j}\left(f_{j}\right)=\left(0, D t_{k j}\left(h_{j}\right) f_{j}^{h} v_{j}\right)+\mathcal{F}_{k j}\left(f_{j}\right)
$$

where $\mathcal{L}_{k j}\left(f_{j}\right)$ stands for $\left(\mathcal{L}_{k j}^{h}\left(f_{j}^{h}\right), \mathcal{L}_{k j}^{v}\left(f_{j}^{v}\right)\right)$ as defined by $(2.16),(2.18)$ :

$$
\begin{aligned}
& \mathcal{L}_{k j}^{h}\left(f_{j}^{h}\right):=f_{k}^{h}\left(\varphi_{k j}\left(h_{j}\right), t_{k j}\left(h_{j}\right) v_{j}\right)-s_{k j}\left(h_{j}\right) f_{j}^{h}\left(h_{j}, v_{j}\right), \\
& \mathcal{L}_{k j}^{v}\left(f_{j}^{v}\right):=f_{k}^{v}\left(\varphi_{k j}\left(h_{j}\right), t_{k j}\left(h_{j}\right) v_{j}\right)-t_{k j}\left(h_{j}\right) f_{j}^{v}\left(h_{j}, v_{j}\right) .
\end{aligned}
$$

Recall that $s_{k j}\left(h_{j}\right)=D \varphi_{k j}\left(h_{j}\right)$ is the Jacobian matrix of $\varphi_{k j}$. Furthermore, we have the horizontal error term

$$
\begin{aligned}
\mathcal{F}_{k j}^{h}\left(f_{j}\right):= & \phi_{k j}^{h}\left(h_{j}+f_{j}^{h}, v_{j}+f_{j}^{v}\right)-\hat{\phi}_{k j}^{h} \\
& +\left(f_{k}^{h}\left(\varphi_{k j}, t_{k j}\left(h_{j}\right) v_{j}\right)-f_{k}^{h}\left(\varphi_{k j}+\hat{\phi}_{k j}^{h}, t_{k j}\left(h_{j}\right) v_{j}+\hat{\phi}_{k j}^{v}\right)\right) \\
& +\varphi_{k j}\left(h_{j}+f_{j}^{h}\left(h_{j}, v_{j}\right)\right)-\varphi_{k j}\left(h_{j}\right)-D \varphi_{k j}\left(h_{j}\right) f_{j}^{h}\left(h_{j}, v_{j}\right),
\end{aligned}
$$


as well as the vertical error term

$$
\begin{aligned}
\mathcal{F}_{k j}^{v}\left(f_{j}\right):= & \phi_{k j}^{v}\left(h_{j}+f_{j}^{h}, v_{j}+f_{j}^{v}\right)-\hat{\phi}_{k j}^{v}+D t_{k j}\left(h_{j}\right) f_{j}^{h} f_{j}^{v} \\
& +\left(f_{k}^{v}\left(\varphi_{k j}, t_{k j}\left(h_{j}\right) v_{j}\right)-f_{k}^{v}\left(\varphi_{k j}+\hat{\phi}_{k j}^{h}, t_{k j}\left(h_{j}\right) v_{j}+\hat{\phi}_{k j}^{v}\right)\right) \\
& +\left(t_{k j}\left(h_{j}+f_{j}^{h}\left(h_{j}, v_{j}\right)\right)-t_{k j}\left(h_{j}\right)-D t_{k j}\left(h_{j}\right) f_{j}^{h}\right)\left(v_{j}+f_{j}^{v}\right) .
\end{aligned}
$$

We collect $2 m$ jets from $(5.17),(5.20),(5.21)$. Since $f_{j}=O(m+1)$ and $\hat{\phi}_{k j}^{\bullet}=O(2 m+1)$, this gives us

$$
\begin{aligned}
{\left[\left(\delta^{h} f^{h}\right)_{k j}\right] \leq 2 m } & =-\left[\phi_{k j}^{h}\right]^{\leq 2 m} \\
{\left[\left(\delta^{v} f^{v}\right)_{k j}\right]^{\leq 2 m} } & =-D t_{k j}\left(h_{j}\right)\left[f_{j}^{h}\right]^{\leq 2 m-1} v_{j}-\left[\phi_{k j}^{v}\right]^{\leq 2 m} .
\end{aligned}
$$

Under formal assumptions, according to Lemma 2.16 (c), equations (5.22)-(5.23) have a solution $\left(\left[f_{j}^{h}\right]_{m+1}^{2 m},\left[f_{j}^{v}\right]_{m+1}^{2 m}\right)$.

We first consider the case that $H^{0}\left(C, \oplus_{k=2}^{2 m} T C \otimes S^{k}\left(N_{C}^{*}\right)\right)=0$. Then, for any $r_{*}<r^{\prime \prime}<$ $r^{\prime}<\tilde{r}<r^{*}$ with

$$
r^{\prime \prime}=\theta r^{\prime}=\theta^{2} r, \quad r^{\prime}-r^{\prime \prime}<r^{*}-\tilde{r},
$$

the solution to (5.22) is unique and by Theorem A.12 that unique solution satisfies the estimate

$$
\left|\left\{\left[f_{k}^{h}\right]^{l}\right\}\right|_{r^{\prime}} \leq \frac{D\left(T C \otimes S^{l}\left(N_{C}^{*}\right)\right)}{\left(r-r^{\prime}\right)^{\tau}}\left|\left\{\left[\phi_{k j}^{h}\right]^{l}\right\}\right|_{r}, \quad l=m+1, \ldots, 2 m .
$$

In particular, $\left\{\left[f_{k}^{h}\right]_{m+1}^{2 m}\right\}$ has been determined. The solvability of (5.23) and Theorem A.12 imply that we can find a solution $\left\{\left[f_{k}^{v}\right]_{m+1}^{2 m}\right\}$ such that for $l=m+1, \ldots, 2 m$,

$$
\left|\left\{\left[f_{k}^{v}\right]^{l}\right\}\right|_{r^{\prime \prime}} \leq \frac{D\left(N_{C} \otimes S^{l}\left(N_{C}^{*}\right)\right)}{\left(r^{\prime}-r^{\prime \prime}\right)^{\tau}}\left\{c \frac{D\left(T C \otimes S^{l-1}\left(N_{C}^{*}\right)\right)}{\left(r-r^{\prime}\right)^{\tau}}\left|\left\{\left[\phi_{k j}^{h}\right]^{l-1}\right\}\right|_{r}+\left|\left\{\left[\phi_{k j}^{v}\right]^{l}\right\}\right|_{r}\right\} .
$$

Here $c$ depends only on the $D t_{k j}$ over the initial covering.

If $H^{0}\left(C, \oplus_{k=m+1}^{2 m} T C \otimes S^{k}\left(N_{C}^{*}\right)\right) \neq 0$, we are in the flat case, that is $D t_{k j}=0$. Thus, we can find a solution $\left\{\left[f_{k}^{v}\right]_{m+1}^{2 m}\right\}$ such that for $l=m+1, \ldots, 2 m$,

$$
\left|\left\{\left[f_{k}^{v}\right]^{l}\right\}\right|_{r^{\prime \prime}} \leq \frac{D\left(N_{C} \otimes S^{l}\left(N_{C}^{*}\right)\right)}{\left(r^{\prime}-r^{\prime \prime}\right)^{\tau}}\left|\left\{\left[\phi_{k j}^{v}\right]^{l}\right\}\right|_{r}
$$

Let us set

$$
D_{*}(2 m):=1+\max _{2 \leq l \leq 2 m}\left\{\left(1+c K\left(T C \otimes S^{l-1}\left(N_{C}^{*}\right)\right)\right) D\left(N_{C} \otimes S^{l}\left(N_{C}^{*}\right)\right\} .\right.
$$

Hence, in any case, estimates (5.24)-(5.26) lead to

$$
\left|\left\{\left[f_{k}^{\bullet}\right]^{l}\right\}\right|_{\theta^{2} r} \leq \frac{C_{1} D_{*}(2 m)}{\left(r-\theta^{2} r\right)^{2 \tau}}\left|\left\{\left[\phi_{k j}^{\bullet}\right]^{l}\right\}\right|_{r}
$$

for all $\theta$ and $r$ satisfying $r_{*} \leq \theta^{2} r<r<\tilde{r}<r^{*}$ and all $m+1 \leq l \leq 2 m$. Assume further that $\theta^{6} r>r_{*}$ and $\left(1-\theta^{7}\right) r<r^{*}-\tilde{r}$. We obtain, by Proposition 5.4 with $\tilde{m}=2 m+1$

$$
\left|\hat{\phi}_{k j}^{\bullet}\right|_{\theta^{7} r} \leq \frac{C_{1} D_{*}(2 m) \theta^{2 m+1}}{\left(r-\theta^{2} r\right)^{2 \tau}}\left|\phi_{k j}^{\bullet}\right|_{r} \leq \theta^{2 m+1}(1-\theta) r / C_{0}
$$


provided

$$
\begin{gathered}
\left|\left\{\phi_{k j}^{\bullet}\right\}\right|_{r} \leq(1-\theta) r / C_{0}, \\
\frac{D_{*}(2 m)}{\left(r-\theta^{2} r\right)^{2 \tau}}\left|\left\{\phi_{k j}^{\bullet}\right\}\right|_{r} \leq(1-\theta) r / C_{0} .
\end{gathered}
$$

Note that condition (5.28) follows from (5.29) as $D_{*}(\ell) \geq 1$.

Rename $\Phi_{k j}, \phi_{k j}^{\bullet}, F_{j}, f_{j}^{\bullet}, \hat{\Phi}_{k j}, \hat{\phi}_{k j}^{\bullet}$ respectively as $\Phi_{k j}^{(0)}, \phi_{k j}^{(0)}, F_{j}^{(0)}, f_{j}^{(0)}, \Phi_{k j}^{(1)}, \phi_{k j}^{(1)}$. Thus $\Phi_{k j}^{(1)}=$ $\left(F_{k}^{(0)}\right)^{-1} \Phi_{k j}^{(0)} F_{j}^{(0)}$. Repeating this formally, we obtain

$$
\Phi_{k j}^{(\ell+1)}=\left(F_{k}^{(\ell)}\right)^{-1} \Phi_{k j}^{(\ell)} F_{j}^{(\ell)}, \quad F_{j}^{(\ell)}=I+f_{j}^{(\ell)}, \quad \Phi_{k j}^{(\ell+1)}=N_{k j}+\phi_{k j}^{(\ell+1)} .
$$

Set $r_{\ell+1}=\theta_{\ell}^{7} r_{\ell}$ and $m_{\ell}=2^{\ell}$. We also have

$$
\begin{gathered}
F_{j}^{(\ell)}\left(\hat{V}_{j}^{r_{\ell+1}}\right) \subset \hat{V}_{j}^{r_{\ell}}, \\
\left|\phi_{k j}^{(\ell+1)}\right|_{r_{\ell+1}} \leq \theta_{\ell}^{2 m_{\ell}+1}\left(1-\theta_{\ell}\right) r_{\ell} / C_{0}
\end{gathered}
$$

provided

$$
\begin{gathered}
r_{*} \leq \theta_{\ell}^{7} r_{k}<1, \quad 0<\theta_{k}<1 ; \\
\frac{C_{1} D_{*}\left(2 m_{\ell}\right)}{\left(r_{\ell}-\theta_{\ell}^{2} r_{\ell}\right)^{2 \tau}}\left|\left\{\phi_{k j}^{(\ell)}\right\}\right|_{r_{\ell}} \leq\left(1-\theta_{\ell}\right) r_{\ell} / C_{0} .
\end{gathered}
$$

To set parameters, we follow Russmann [35]; see [4,39] for different choices of parameters. As in [35], we now use an addition assumption that

$$
D_{\ell}^{*} \geq \ell, \quad \ell \geq 1
$$

Indeed, when $\tilde{D}_{*}(k)=\max \left(D_{*}(k), k\right)$ replaces with $D_{*}(k)$, the sequence $D_{*}(k)$ still increases and $\sum 2^{-k} \log D_{*}\left(2^{k}\right)$ converges. For a constant $C_{*} \geq 1$ to be determined later, define

$$
\begin{aligned}
& m_{\ell}=2^{\ell_{0}+\ell}, \quad r_{\ell+1}=\theta_{\ell}^{7} r_{\ell}, \quad r_{0}=1, \\
& 1-\theta_{\ell}=\delta_{\ell}, \quad \delta_{\ell}=C_{*} \frac{\log D_{*}\left(m_{\ell+2}\right)}{m_{\ell+2}} .
\end{aligned}
$$

Note that in [35, Lemma 6.2] and [4,39], $\omega\left(m_{\ell+1}\right)$ is used to define $\delta_{\ell}$. Shifting the index by 1 , we use $D_{*}\left(m_{\ell+2}\right)$ to simplify the argument. We can find $\ell_{0}=\ell_{0}\left(C_{*}\right)$ such that $0<\theta_{\ell}<1$ for all $\ell$ and furthermore

$$
\prod_{\ell=0}^{\infty} \theta_{\ell}^{7}=\prod_{\ell=0}^{\infty}\left(1-\delta_{\ell}\right)^{7} \geq \exp \left\{-\sum_{\ell=0}^{\infty} \frac{7 C_{*}}{2} \frac{\log D_{*}\left(m_{\ell+2}\right)}{m_{\ell+2}}\right\} .
$$

Since $\sum 2^{-k} \log D_{*}\left(2^{k}\right)<\infty$, the latter is larger than $r_{*}$, provided $\ell_{0}>\ell_{0}\left(C_{*}\right)$. Inductively, we want to show that if $(5.33)_{\ell}$ holds, then $(5.33)_{\ell+1}$ also holds. Indeed, with $(5.33)_{\ell}$, we 
can use $(5.31)_{\ell+1}$ to obtain

$$
\begin{gathered}
\frac{C_{1} D_{*}\left(m_{\ell+2}\right)}{\left(r_{\ell+1}-\theta_{\ell+1}^{2} r_{\ell+1}\right)^{2 \tau}}\left|\left\{\phi_{k j}^{\ell+1}\right\}\right|_{r_{\ell+1}} \times \frac{C_{0}}{\left(1-\theta_{\ell+1}\right) r_{\ell+1}} \\
\leq \frac{D_{*}\left(m_{\ell+2}\right) \theta_{\ell}^{2 m_{\ell}-6}}{\left(r_{\ell+1}-\theta_{\ell+1}^{2} r_{\ell+1}\right)^{2 \tau}} \times \frac{1-\theta_{\ell}}{1-\theta_{\ell+1}} \quad(\text { by }(5.31)) \\
\leq \frac{C_{2} D_{*}\left(m_{\ell+2}\right) \theta_{\ell}^{2 m_{\ell}-6}}{\left(1-\theta_{\ell+1}\right)^{2 \tau+1}}=\frac{C_{2} D_{*}\left(m_{\ell+2}\right)\left(1-\delta_{\ell}\right)^{2 m_{\ell}-6}}{\delta_{\ell+1}^{2 \tau+1}} .
\end{gathered}
$$

We need to check that the last expression is less than one by using logarithm. Note that

$$
\log (1-\delta)<-\delta, \quad \forall \delta \in(0,1)
$$

Therefore,

$$
\begin{aligned}
\log & \frac{C_{2} D_{*}\left(m_{\ell+2}\right)\left(1-\delta_{\ell}\right)^{2 m_{\ell}-6}}{\delta_{\ell+1}^{2 \tau+1}}<\log C_{2}-\left(2 m_{\ell}-6\right) \delta_{\ell}+\log D_{*}\left(m_{\ell+2}\right)-(2 \tau+1) \log \delta_{\ell+3} \\
= & \log C_{2}-\left(2 m_{\ell}-6\right) C_{*} \frac{\log D_{*}\left(m_{\ell+2}\right)}{m_{\ell+2}}+\log D_{*}\left(m_{\ell+2}\right)-(2 \tau+1) \log \left(C_{*} \frac{\log D_{*}\left(m_{\ell+3}\right)}{m_{\ell+3}}\right) \\
= & \left\{\log C_{2}-\frac{\left(2 m_{\ell}-6\right) C_{*}}{3} \frac{\log D_{*}\left(m_{\ell+2}\right)}{m_{\ell+2}}\right\}+\left\{\log D_{*}\left(m_{\ell+2}\right)-\frac{\left(2 m_{\ell}-6\right) C_{*} \log D_{*}\left(m_{\ell+2}\right)}{m_{\ell+2}}\right\} \\
& +\left\{-\frac{\left(2 m_{\ell}-6\right) C_{*}}{3} \frac{\log D_{*}\left(m_{\ell+2}\right)}{m_{\ell+2}}-(2 \tau+1) \log \left(C_{*} \frac{\log D_{*}\left(m_{\ell+3}\right.}{m_{\ell+3}}\right)\right\} .
\end{aligned}
$$

When $\ell_{0}$ is sufficiently large, then $m_{\ell+2}>24$. This implies that if $C_{*}>12$, the sum in each of first two braces is negative. Since log increases, we have by (5.34)

$$
\begin{gathered}
-\log D_{*}\left(m_{\ell+3}\right) \leq \log \frac{1}{m_{\ell+3}} \\
-\log \left(C_{*} \frac{\log D_{*}\left(m_{\ell+3}\right)}{m_{\ell+3}}\right) \leq-\log \left(-C_{*} \frac{\log \frac{1}{m_{\ell+3}}}{m_{\ell+3}}\right) .
\end{gathered}
$$

With $m_{\ell}>6$, the difference in the last brace is bounded above by

$$
\frac{\left(2 m_{\ell}-6\right) C_{*}}{3} \frac{\log \frac{1}{m_{\ell+2}}}{m_{\ell+2}}-(2 \tau+1) \log \left(C_{*} \frac{\log m_{\ell+3}}{m_{\ell+3}}\right) \leq\left(-\frac{1}{12} C_{*}+2 \tau+1\right) \log m_{\ell+2},
$$

which is negative when $C_{*}>24 \tau+12$. We have determined $C_{*}$. This allows us to determine $\ell_{0}\left(C_{*}\right)$ so that $0<\theta_{\ell}<1$ and $\prod_{\ell=0}^{\infty} \theta_{\ell}^{7}>r_{*}$. Therefore, (5.33) $)_{\ell}$ holds if it holds for initial value $\ell=0$. Using a dilation $v_{j} \rightarrow \epsilon v_{j}$ for $\epsilon>0$, we may replace $\Phi_{k j}\left(h_{j}, v_{j}\right)$ by $\left(\varphi_{k j}\left(h_{j}\right)+\phi_{k j}^{h}\left(h_{j}, \epsilon v_{j}\right), t_{k j}\left(h_{j}\right) v_{j}+\epsilon^{-1} \phi_{k j}^{v}\left(h_{j}, \epsilon v_{j}\right)\right)$. This yields $(5.33)_{0}$ when $\epsilon$ is sufficiently small, as $\phi_{k j}^{\bullet}\left(h_{j}, v_{j}\right)=O\left(\left|v_{j}\right|^{2}\right)$.

To finish the proof, we set $\Psi_{j}^{(\ell)}:=F_{j}^{(0)} \circ \cdots \circ F_{j}^{(\ell)}$. We have

$$
\Psi_{j}^{(\ell)}\left(\hat{V}_{j}^{r_{\ell+1}}\right) \subset \hat{V}_{j}^{r_{\ell}}, \quad \Psi_{j}^{(\ell+1)}\left(h_{j}, v_{j}\right)-\Psi_{j}^{(\ell)}\left(h_{j}, v_{j}\right)=O\left(\left|v_{j}\right|^{\ell}\right) .
$$


Consequently, the sequence $\Psi_{j}^{(\ell)}$ is bounded in $\hat{V}_{j}^{r_{\infty}}$. Fix $0<\theta<1$. By the Schwarz lemma, we get

$$
\sup _{\hat{U}_{j}^{r \infty} \times B_{d}^{\theta r_{\infty}}}\left|\Psi_{j}^{(\ell+1)}-\Psi_{j}^{(\ell)}\right| \leq C \theta^{\ell} .
$$

Therefore, of $\Psi_{j}^{(\ell)}$ converges uniformly on $\hat{U}_{j}^{r_{\infty}} \times B_{d}^{\theta r_{\infty}}$ to a holomorphic mapping $\Psi_{j}^{\infty}$. Then $F:=N_{j}^{-1} \Psi_{j}^{\infty} \Phi_{j}$ is well defined. Indeed, $N_{k}^{-1} \Psi_{k}^{\infty} \Phi_{k}=N_{j}^{-1} \Psi_{j}^{\infty} \Phi_{j}$ is equivalent to $\Psi_{k}^{\infty}\left(\Phi_{k} \Phi_{j}^{-1}\right)=\left(N_{k} N_{j}^{-1}\right) \Psi_{j}^{\infty}$. Since $\Psi_{j}^{\infty}$ are tangent to the identity, they are germs of biholomorphisms. Therefore, $F$ linearizes a small neighborhood of $C$ in $M$.

Therefore, we have proved the following full linearization result.

Theorem 5.5. Let a neighborhood of the compact manifold $C$ in $M$ be equivalent to a neighborhood of the zero section of normal bundle $N_{C}$ of $C$ in $M$ by a formal holomorphic mapping which is tangent to the identity and preserves the splitting of $T_{C} M$. Assume that $H^{0}\left(C, T C \otimes S^{\ell}\left(N_{C}^{*}\right)\right)=0$ for all $\ell>1$ or that the normal bundle $N_{C}$ is flat. If $\left\{D_{*}\left(2^{k}\right)\right\}$ defined by (5.1) and (5.27) satisfies

$$
\sum_{k \geq 1} \frac{\log D_{*}\left(2^{k+1}\right)}{2^{k}}<+\infty,
$$

there is a neighborhood of the compact manifold $C$ in $M$ that is biholomorphic to a neighborhood of the zero section of normal bundle of $C$ in $M$.

When $C$ is affine and $N_{C}$ is flat, the formal equivalence assumption can be relaxed by assuming that the neighborhoods are equivalent under a formal biholomorphisms fixing $C$ pointwise. This follows from Lemma $2.4(c)$.

We now present two examples to illustrate the results in this paper.

5.3. An example of Arnol'd. This is originally studied by Arnold [2], [3, §27] for linearization of a neighborhood. See also Ilyashenko-Pyartli [22] for linearization for flat tori in higher dimensions.

Example 5.6. [3, §27]. Let $C$ be defined by identifying points in $\mathbf{C}$ via

$$
h=0 \bmod (2 \pi, 2 \omega), \quad h \in \mathbf{C},
$$

where $\omega=a+i b$ with $b>0$ and $a \geq 0$. Consider domains in $C$ defined by parallelograms

$$
\begin{gathered}
U_{1}=P(-r \pi-r \omega,(1+r) \pi-r \omega,(1+r) \pi+(1+r) \omega,-r \pi+(1+r) \omega) \\
U_{4}=U_{1}+\pi, \quad U_{3}=U_{4}+\omega, \quad U_{2}=U_{3}-\pi .
\end{gathered}
$$

Suppose that $r>0$ is sufficiently small. Then $U_{i} \cap U_{j}$ has two connected components $U_{i j, 0}$ and $U_{i j, 1}$ with

$$
U_{14,1}=U_{14,0}-\pi, \quad U_{34,1}=U_{34,0}-\omega, \quad U_{23,1}=U_{23,0}-\pi, \quad U_{12,1}=U_{12,0}-\omega .
$$

Let $\hat{U}_{j}=U_{j}$ and $\hat{V}_{j}=\hat{U}_{j} \times \Delta_{\delta}$. Define $M=\cup \hat{V}_{j} / \sim, V_{j}=\left\{[x]: x \in \hat{V}_{j}\right\}, \Phi_{j}: V_{j} \rightarrow \hat{V}_{j}$ and the transition functions $\Phi_{k j}$ on $V_{k j}=V_{k} \cap V_{j}$ of $M$ as follows. Let

$$
f(h, v)=(h+2 \omega+v b(h, v), \lambda v(1+v a(h, v))), \quad|\operatorname{Im} h|<\delta
$$


where $a, b$ are $2 \pi$ periodic holomorphic function in $h$. Define

$$
\begin{array}{r}
\Phi_{12,0}=\mathrm{I}, \quad \Phi_{43,0}=\mathrm{I}, \quad \Phi_{12,1}=\left.f\right|_{\hat{V}_{12}}, \quad \Phi_{43,1}=\left.f\right|_{\hat{V}_{43}}, \\
\Phi_{14}=\mathrm{I}, \quad \Phi_{23}=\mathrm{I}, \\
\Phi_{13,0}=\mathrm{I}, \quad \Phi_{13,1}=\left.f\right|_{\hat{V}_{13,1}}, \quad \Phi_{42,0}=\mathrm{I}, \quad \Phi_{42,1}=\left.f\right|_{\hat{V}_{42,1}} .
\end{array}
$$

The linearization of a neighborhood of $C$ in $M$ is equivalent to $G_{k}^{-1} \Phi_{k j} G_{j}=\hat{\Phi}_{k j}$ where $\hat{\Phi}_{k j}$ are constructed as above by replacing $f$ with $\hat{f}$ defined by

$$
\hat{f}(h, v)=(h+2 \omega, \lambda v) .
$$

Thus $T M$ has transition functions:

$$
\hat{\Phi}_{14}=\mathrm{I}, \quad \hat{\Phi}_{23}=\mathrm{I}, \quad \hat{\Phi}_{12,0}=\mathrm{I}, \quad \hat{\Phi}_{43,0}=\mathrm{I}, \quad \hat{\Phi}_{12,1}=\left.\hat{f}\right|_{\hat{V}_{12}}, \quad \hat{\Phi}_{43,1}=\left.\hat{f}\right|_{\hat{V}_{43}} .
$$

Then we have $g:=G_{1}=G_{4}$ on $\hat{V}_{1} \cap \hat{V}_{4}, g:=G_{2}=G_{3}$ on $\hat{V}_{2} \cap \hat{V}_{3}, g:=G_{1}=G_{2}$ on $\hat{V}_{12,0}$ and $g:=G_{3}=G_{4}$ on $\hat{V}_{34,0}$. In other words, $g$ is $2 \pi$ periodic and defined on $-\delta \operatorname{Im} \omega<\operatorname{Im} h<2(1+\delta) \operatorname{Im} \omega$. The cohomology equation is reduced to $G_{1}^{-1} \Phi_{12} G_{2}=\hat{\Phi}_{12}$ and $G_{4}^{-1} \Phi_{43} G_{3}=\hat{\Phi}_{43}$. Equivalently, we need to solve

$$
g^{-1} f g=\hat{f}
$$

Assume that $f$ has been normalized so that

$$
v a(h, v)=v^{n} a_{n}(h)+O(n+1), \quad v b(h, v)=v^{n} b_{n}(h)+O(n+1), \quad n=1,2, \ldots
$$

For the purpose of illustration, we will only restrict to a special unitary line bundle case where $|\lambda|=1$. Then by the non-resonance condition that $\lambda$ is not a root of unity, we may assume that as in $[3$, p. 211]

$$
g(h, v)=\left(h+v^{n} B_{n}(h), v\left(1+v^{n} A_{n}(h)\right)+O(n+1) .\right.
$$

This leads to decoupled equations of the form

$$
\begin{aligned}
& \lambda^{n} A_{n}(h+2 \omega)-A_{n}(h)=-a_{n}(h), \\
& \lambda^{n} B_{n}(h+2 \omega)-B_{n}(h)=-b_{n}(h) .
\end{aligned}
$$

Note that $a_{n}, b_{n}$ are holomorphic in $|\operatorname{Im} h|<\delta$ and we are seeking a solution on a large strip

$$
-\delta^{\prime}<\operatorname{Im} h<\operatorname{Im} \omega+\delta^{\prime} .
$$

In Fourier coefficients $a_{n, \ell}$ and a non-resonant condition, the Fourier coefficients of $A_{n}$ are given by

$$
A_{n, j}=\frac{a_{n, j}}{\lambda^{n} e^{2 \omega j \sqrt{-1}}-1} .
$$

Assume that $a_{n}$ are holomorphic and $2 \pi$ periodic in $h$ for $S_{\delta}:|\operatorname{Im} h|<\delta$. Suppose that

$$
\left|\lambda^{n} e^{2 j \omega \sqrt{-1}}-1\right| \geq c\left|\lambda^{n}-1\right|
$$


Then

$$
\begin{gathered}
\left|A_{n, j}\right| \leq \frac{C}{\left|\lambda^{n}-1\right|}\left|a_{n}\right|_{L^{2}\left(S_{\delta}\right)} e^{-|j| \delta}, \\
\left|A_{n, j} e^{j h}\right| \leq \frac{C}{\left|\lambda^{n}-1\right|}\left|a_{n}\right|_{L^{2}\left(S_{\delta}\right)} e^{-|j|\left(\delta-\delta^{\prime}\right)}, \quad-\delta^{\prime}<\operatorname{Im} h<\operatorname{Im} \omega+\delta^{\prime} .
\end{gathered}
$$

Furthermore, we can verify that

$$
\left|A_{n}\right|_{L^{2}\left(S_{\delta^{\prime}}\right)} \leq \frac{C}{\left(\delta-\delta^{\prime}\right)\left|\lambda^{n}-1\right|}\left|a_{n}\right|_{L^{2}\left(S_{\delta}\right)}
$$

Note that $t_{k j}$ are locally constant with values $1, \lambda, \lambda^{-1}$.

Therefore, we have verified

$$
D\left(\left(T_{C} \oplus N_{C}\right) \otimes S^{n} N_{C}^{*}\right) \leq \frac{C}{\left|\lambda^{n}-1\right|} .
$$

By Lemma A.2, we get an estimate with equivalent bounds (up to a scalar) but in the original domain, i.e. without shrinking domains.

Strictly speaking, the above covering $\left\{U_{j}^{r}\right\}$ has non smooth boundary. The intersection is non-transversal either. However, this covering can be easily modified to get a generic covering defined early, replacing $\hat{U}_{j}$ by smooth strictly convex domains $\hat{U}_{j}$ and then replacing $\hat{U}_{j}$ by $\hat{U}_{j}+c_{j}$ for suitable small constants.

5.4. Counter-examples. We now show that a certain small-condition is necessary to ensure the vertical and full linearizations. We will achieve this by establishing a connection between the classical linearization problem for germs of one-dimensional holomorphic mappings and the vertical linearization of foliated neighborhood of an elliptic curve.

We keep the notation in subsecton 5.3. Let us start with a power series

$$
a(h, v)=\sum_{n \geq 2} a_{n} v^{n}:=a(v) .
$$

Set $b(h, v)=0$. Then we have a neighborhood of $C$ associated to

$$
f(h, v)=(h+2 \omega, \lambda v+a(v)) .
$$

Since the vertical part of the transition functions depends only on $v$, then $M$ is already admits a horizontal foliation with center $C$ being compact.

Proposition 5.7. Let $M_{\lambda, \omega, a}$ be neighborhood of $C$ defined by transition functions $\Phi_{k j}$ given by (5.36)-(5.38) where $f$ is given by (5.41)-(5.42). Suppose that $\lambda, \omega$ satisfy the nonresonant condition

$$
\lambda^{n} e^{2 j \omega \sqrt{-1}}-1 \neq 0, \quad n=2,3, \ldots, j \in \mathbf{Z} .
$$

Then $M_{\lambda, \omega, a}$ is vertically (resp. formally) linearizable by a mapping tangent to the identity if and only if the germ of holomorphic mapping $\varphi(v)=\lambda v+a(v)$ is holomorphically (resp. formally) linearizable.

Proof. Suppose that $M$ is vertically linearizable by a holomorphic mapping that is tangent to the identity. By Proposition 2.6, it is vertically linearization by a mapping $G_{j}$ such that

$$
G_{j}\left(h_{j}, v_{j}\right)=\left(h_{j}, v_{j}+O\left(\left|v_{j}\right|^{2}\right)\right) .
$$


By the non-resonant condition (5.43), we can verify that (5.39) is equivalent to that the $g$ in (5.39) has the form $g(h, v)=(h, \psi(v))$ and $\varphi$ is linearized by $\psi$.

The existence of non-holomorphically linearizable $\varphi$ is well-known. By theorems of Bruno [4] and Yoccoz [41], Proposition 5.7 shows that $M_{\lambda, \omega, \hat{a}}$ with $\hat{a}(v)=v^{2}$ is vertically linearizable and hence linearizable if and only if $\lambda$ is a Bruno number, that is

$$
\sum_{k \geq 1} \frac{\log \max _{2 \leq j \leq 2^{k}}\left|\lambda^{j}-1\right|^{-1}}{2^{k}}<+\infty .
$$

5.5. A foliation example. Here we specialize Ueda's theory for elliptic curves. Let us first discuss the Fischer norms and Bergman norm when the $N_{C}$ is unitary. Let us recall two formulae from Zhu [42, p. 22]:

$$
\begin{gathered}
\int_{\partial B_{r}^{d}}\left|z^{Q}\right|^{2} d \sigma_{d}=\frac{(d-1) ! Q !}{(|Q|+d-1) !} r^{2 d-1+2|Q|} \\
\int_{B_{r}^{d}}\left|z^{Q}\right|^{2} d V_{d}=\frac{d ! Q !}{(|Q|+d) !} r^{2|Q|+d} .
\end{gathered}
$$

Therefore, there is a precise asymptotic behavior of Fischer norm and the Bergman norm:

$$
c_{d}\|g\|_{L^{2}\left(B_{r}^{d}\right)}^{2} \leq|g|_{f, r}^{2} \leq C_{d}\|g\|_{L^{2}\left(B_{r}^{d}\right)}^{2}, \quad 1 / 4<r<4 .
$$

We also have Bergman's inequality for $L^{2}$ holomorphic functions [14, p. 189]:

$$
\begin{gathered}
|f|_{\infty, \hat{V}_{j}^{(1-\theta) r}} \leq \frac{C_{d}}{(\theta r)^{d}} \sup _{h_{j}}\left|f\left(h_{j}, \cdot\right)\right|_{L^{2}\left(B_{r}^{d}\right)}, \\
\sup _{h_{j}}\left|f\left(h_{j}, \cdot\right)\right|_{L^{2}\left(B_{r}^{d}\right)} \leq C_{d}|f|_{\infty, B_{r}^{d}}, \quad 1 / 4<r<4 .
\end{gathered}
$$

In general, we get

$$
\begin{gathered}
\left|\phi_{k j}^{\bullet}\right|_{L^{\infty}\left(\hat{V}_{k j,(1-\theta) r}\right)} \leq \frac{C_{d}}{(\theta r)^{d}} \sup _{h_{j}}\left|\phi_{k j}^{\bullet}\left(h_{j}, \cdot\right)\right|_{L^{2}\left(B_{k j, r}^{d}\left(h_{j}\right)\right)}, \\
\sup _{h_{j}}\left|\phi_{k j}^{\bullet}\right|_{L^{2}\left(B_{k j, r}^{d}\left(h_{j}\right)\right)} \leq C_{d}\left|\phi_{k j}^{\bullet}\right|_{L^{\infty}\left(\hat{V}_{k j}^{r}\right)}, \quad 1 / 4<r<4 .
\end{gathered}
$$

Note that when $t_{k j}$ are unitary, the skewed domain $\hat{V}_{k j}^{r}$ defined in (5.2) are actually product domains

$$
\hat{V}_{k j}^{r}=\hat{U}_{k j}^{r} \times B_{d}^{r}
$$

Therefore, the Fischer norm and Bergman norm bound each other with constants depending only on $\theta$ and $d$. We can fix $\theta$ too by applying Lemma A.2 as we did in sections 3 and 4 . Therefore, any estimate of cohomology equations in Fischer norms has a counter part in super norm on the unit ball in $\mathbf{C}^{d}$ and vice versa.

Note that the small divisors condition

$$
\left|\lambda^{n}-1\right| \geq C n^{-\tau}, \quad n=1,2, \ldots
$$

for some constants $C, \tau$ is equivalent to Ueda's condition in terms of $\operatorname{dist}\left(N_{C}^{n}, 1\right)$ for the foliation problem when $C$ is an elliptic curve of type zero. In this case the linearized 
equation corresponds to equation (5.40) we can take the small divisor $1 / K_{*}\left(N_{C} \otimes S^{n} N_{C}^{*}\right)$ to be $\left|\lambda^{n}-1\right|$.

Finally, we should mention that the assumption $\eta_{m} \leq L_{0} L^{m}$ is satisfied under Siegel's small divisor condition $\left|\lambda^{n}-1\right| \geq C n^{-\tau}$ by a method of Siegel; see Ueda [40] for the vertical linearization problem. It is also satisfied under the Bruno condition [4] which is a condition weaker than (5.49). For the details, we refer to $[4,34]$.

\section{Appendix A. $L^{2}$ BOUnds of COHOMOLOGY SOLUTIONS AND SMALL DIVISORS}

A.1. A question of Donin. Let $E$ be a holomorphic vector bundle on a compact complex manifold $C$. The main purpose of this section is to obtain $L^{2}$ and sup-norm bounds for the cohomology equation

$$
\delta u=f
$$

where $f \in Z^{1}(\mathcal{U}, \mathcal{O}(E))$ and $\mathcal{U}$ is a suitable covering of $C$. Our goal is to show that if $f=0$ in $H^{1}(C, \mathcal{O}(E))$, then there is a solution $u$ such that

$$
\|u\|_{\mathcal{U}} \leq K(E)\|f\|_{\mathcal{U}}
$$

Here $\|\cdot\|_{\mathcal{U}}$ is the $L^{2}$-norm for cochains of the covering $\mathcal{U}$. The main assertion is that the solution $u$ admits estimate on the original covering $\mathcal{U}$ without any refinement, which is important to the application in this paper. For this purpose, we will choose the covering $\mathcal{U}$ which consists of biholomorphic images of the unit polydisc, which are in the general position. The question on the existence of such an estimate and solutions was raised by Donin who asked the general question if $\mathcal{O}(E)$ is replaced by a coherent analytic sheaf $\mathcal{F}$ on $C$ and $f$ is any $p$-cocycle, with $p>0$, of a covering $\mathcal{U}$ [8]. The result in this appendix provides an affirmative answer to Donin's question for $p=1$ and the sheaf of holomorphic sections of a holomorphic vector bundle. Furthermore, we will introduce the small divisor for (A.1) in (A.2). Although some of results in this appendix can be further developed for a general setting, we limit to the case of $H^{1}\left(C, \mathcal{O}\left(E^{\prime} \otimes E^{\prime \prime}\right)\right)$; this suffices applications in this paper. One may take $E^{\prime \prime}$ to be the trivial bundle to deal with a general vector bundle $E$. In the applications we have in mind, $C$ is embedded into a complex manifold $M$ and we will take $E^{\prime \prime}$ to be symmetric powers $\operatorname{Sym}^{\ell} N_{C}^{*}$ of $N_{C}^{*}$, the dual of the normal bundle of $C$ in $M$. In this paper, $S^{\ell} E$ denotes the symmetric power $\operatorname{Sym}^{\ell} E$ of a vector bundle $E$ over $C$. We are mainly concerned with how various bounds depend on $\ell$ as $\ell \rightarrow \infty$ when we employ the important Fisher metric on $S^{\ell} N_{C}^{*}$ for unitary the normal bundle $N_{C}$. This will be crucial in our applications.

To prove (A.2), we will first use the original estimate of Donin [8], without solving the cohomology equation. This serves as a smoothing decomposition in the sense of Grauert [14] by expressing

$$
f=g+\delta u
$$

where $g$ is defined on a larger covering while $u$ is defined on a shrinking covering. We will then combine with the proof of finiteness theorem of cohomology groups from GrauertRemmert [14] to refine the decomposition (A.3) by expressing $g$ in a base of cocycles. Finally, we will obtain (A.2) by avoiding shrinking of covering. This last step is motivated by a method of Kodaira-Spencer and Ueda [40]. We take a different approach by an 
essential use of the uniqueness theorem. This allows us to introduce the small divisors in (A.2) to the cohomology equation (A.1).

A.2. Bounds of solutions of cohomology equations. We now start to introduce nested coverings of $C$. This will be an essential ingredient of the small divisors for the cohomology equation. We cover $C$ by finitely many open sets $U_{i}, i \in \mathcal{I}$ such that there are open sets $V_{i}$ in $M$ with $V_{i} \cap C=U_{i}$. We also assume that there are biholomorphic mappings $\Phi_{i}$ from $V_{i}$ onto the polydisc $\Delta_{n+d}^{r^{*}}$ of radius $r^{*}$, where $n$ is the dimension of $C$ and $n+d$ is the dimension of $M$. Assume further that $\Phi_{i}\left(U_{i}^{r^{*}}\right)=\Delta_{n}^{r^{*}} \times\{0\}$ for $\varphi_{i} \times\{0\}=\left.\Phi_{i}\right|_{U_{i}}$. Set $\mathcal{U}^{r}=\left\{U_{i}^{r}: i \in \mathcal{I}\right\}$ with $U_{i}^{r}=\varphi_{i}^{-1}\left(\Delta_{n}^{r}\right)$. We assume that $r^{*}<1$ and $\mathcal{U}^{r_{*}}$ with $r_{*}<r^{*}$, remains a covering of $C$. When $U_{I}^{r}:=U_{i_{0}}^{r} \cap \cdots U_{i_{q}}^{r}$ is non-empty, it is still Stein [14, p. 127].

Definition A.1. Let $\left\{U_{j}^{r}\right\}$ be an open covering of $C$ for each $r \in\left[r_{*}, r^{*}\right]$. We say that the family of coverings $\left\{U_{j}^{r}\right\}$ is nested, if each connected component of $U_{k}^{\rho} \cap U_{j}^{r_{*}}$ intersects $U_{k}^{r_{*}} \cap U_{j}^{r_{*}}$ when $r_{*} \leq \rho \leq r^{*}$. In particular, $U_{k}^{r_{*}} \cap U_{j}^{r_{*}}$ is non-empty if and only if $U_{k}^{\rho} \cap U_{j}^{r_{*}}$ is non-empty.

Let $N\left(U_{i}^{r^{*}}\right)$ be the union of all $\overline{U_{k}^{r^{*}}}$ that intersect $\overline{U_{i}^{r^{*}}}$; as in [8] we will call the union the star of $U_{i}^{r^{*}}$. Refining $\mathcal{U}^{r^{*}}$ if necessary, we may assume that there is a biholomorphism $\varphi_{i}$ from a neighborhood of the star onto an open set in $\mathbf{C}^{n}$. If $E^{\prime}, E^{\prime \prime}$ are holomorphic vector bundles over $C$, we will fix a trivialization of $E^{\prime}$ over $U_{i}$ by fixing a holomorphic basis $e_{k}^{\prime}=\left\{e_{k, 1}^{\prime}, \ldots, e_{k, m}^{\prime}\right\}$ in $\overline{U_{k}^{r^{*}}}$. We also fix a holomorphic base $e_{j}^{\prime \prime}=\left\{e_{j, 1}^{\prime \prime}, \ldots, e_{j, d}^{\prime \prime}\right\}$ of $E^{\prime \prime}$ in $\overline{U_{j}^{r^{*}}}$. On $U_{I}^{r^{*}}=U_{i_{0}}^{r^{*}} \cap \cdots \cap U_{i_{q}}^{r^{*}}$, it will be convenient to use the base

$$
e_{i_{0} \ldots i_{q}}:=e_{i_{0}}^{\prime} \otimes e_{i_{q}}^{\prime \prime}:=\left\{e_{i_{0}, k}^{\prime} \otimes e_{i_{q}, j}^{\prime \prime}: 1 \leq k \leq m, 1 \leq j \leq d\right\}
$$

Throughout the paper $\|\cdot\|_{D}$ and $|\cdot|_{D}$ denote respectively the $L^{2}$ and sup norms of a function in $D$, when $D$ is a domain in $\mathbf{C}^{n}$. If $f=\left(f_{1}, \ldots, f_{d}\right)$ is a vector of functions, we define the $L^{2}$ norm, metric, and sup norms as follows:

$$
\begin{aligned}
\|f\|_{D}^{2} & :=\|f\|_{L^{2}(D)}^{2}:=\left\|f_{1}\right\|_{D}^{2}+\cdots+\left\|f_{d}\right\|_{D}^{2}, \\
|f|_{D}^{2} & :=\sup _{z \in D}\left|f_{1}(z)\right|^{2}+\cdots+\left|f_{d}(z)\right|^{2} \\
|f|_{\infty, D} & :=\sup _{z \in D} \max \left\{\left|f_{1}(z)\right|, \ldots,\left|f_{d}(z)\right|\right\} .
\end{aligned}
$$

For a $d \times d$ matrix $t$ of functions on $D$, denote by $|t|_{D},\|t\|_{D},|t|_{\infty, D}$ respectively the operator norms defined by

$$
|t|_{D}=\sup _{|f|_{D}=1}|t f|_{D}, \quad\|t\|_{D}=\sup _{\|f\|_{D}=1}\|t f\|_{D}, \quad|t|_{\infty, D}=\sup _{|f|_{\infty, D}=1}|t f|_{\infty, D} .
$$

Therefore, $\|t\|_{D} \leq|t|_{D}$ as $\|t f\|_{D} \leq\left(\sup _{z \in D}|t(z)|\right)\|f\|_{D}=|t|_{D}\|f\|_{D}$.

Then we define the $L^{2}$ norm for $f \in C^{q}\left(\mathcal{U}^{r}, \mathcal{O}\left(E^{\prime} \otimes E^{\prime \prime}\right)\right)$ by

$$
\begin{aligned}
a_{I} e_{I} & :=\sum_{\mu=1}^{m d} a_{I}^{\mu} e_{I, \mu}, \\
\|f\|_{\mathcal{U}^{r}} & :=\max _{I=\left(i_{0}, \ldots, i_{q}\right) \in \mathcal{I}^{q+1}}\left\{\left\|a_{I} \circ \varphi_{i_{q}}^{-1}\right\|_{\varphi_{i_{q}}\left(U_{I}\right)}: f_{i}=a_{I} e_{I} \text { in } U_{I}\right\} .
\end{aligned}
$$


Sometimes we denote $\|f\|_{\mathcal{U}^{r_{*}}}$ by $\|f\|$ for abbreviation. We define similarly the metric norm $|f|_{\mathcal{U}^{r *}}$, or $|f|$, and the sup-norm $|f|_{\infty, \mathcal{U}^{r *}}$ or sup $|f|$. It is obvious that

$$
\begin{aligned}
\|f\| \leq C|f|, \quad \sup |f| & \leq\|f\| \leq C \sqrt{\operatorname{rank}\left(E^{\prime} \otimes E^{\prime \prime}\right)} \sup |f|, \\
|t|_{\infty} \leq|t| & \leq C \operatorname{rank}\left(E^{\prime} \otimes E^{\prime \prime}\right)|t|_{\infty},
\end{aligned}
$$

where $C$ does not depend on $E^{\prime}, E^{\prime \prime}$.

The first result of this appendix is to find a way to obtain solutions with bounds to (A.1) on the original covering, if a solution with a bound exists on a shrinking covering. This relies on the nested coverings defined above. We first study the $L^{2}$ norms case.

Lemma A.2. Let $\mathcal{U}^{r}=\left\{U_{i}^{r}: i \in \mathcal{I}\right\}$ with $r_{*} \leq r \leq r^{*}$ be a family of nested finite coverings of $C$. Suppose that $f \in C^{1}\left(\mathcal{U}^{r^{*}}, E^{\prime} \otimes E^{\prime \prime}\right)$ and $f=0$ in $H^{1}\left(\mathcal{U}^{r^{*}}, E^{\prime} \otimes E^{\prime \prime}\right)$. Assume that there is a solution $v \in C^{0}\left(\mathcal{U}^{r_{*}}\right)$ such that

$$
\delta v=f, \quad\|v\|_{\mathcal{U}^{r *}} \leq K\|f\|_{\mathcal{U}^{* *}} .
$$

Then there exists a solution $u \in C^{0}\left(\mathcal{U}^{r^{*}}\right)$ such that $\delta u=f$ on $\mathcal{U}^{r^{*}}$ and

$$
\|u\|_{\mathcal{U}^{r^{*}}} \leq C\left(\left|\left\{t_{k j}^{\prime}\right\}\right|_{\mathcal{U}^{r^{*}}}+K\left|\left\{t_{k j}^{\prime}\right\}\right|_{\mathcal{U}^{r^{*}}}\left|\left\{t_{k j}^{\prime \prime}\right\}\right|_{\mathcal{U}^{r^{*}}}\right)\|f\|_{\mathcal{U}^{r^{*}}}
$$

where $t_{k j}^{\prime}, t_{k j}^{\prime \prime}$ are the transition matrices of $E^{\prime}, E^{\prime \prime}$, respectively, and $C$ depends only on the number $|\mathcal{I}|$ of open sets in $\mathcal{U}^{r^{*}}$ and transition functions of $C$. In particular, $C$ does not depend on $E^{\prime}, E^{\prime \prime}$.

Proof. By assumptions, we have

$$
\begin{gathered}
f_{j k}=(\delta v)_{j k}, \quad U_{j}^{r_{*}} \cap U_{k}^{r_{*}}, \\
\|v\|_{\mathcal{U}^{r_{*}}} \leq K\|f\|_{\mathcal{U}^{r^{*}}}
\end{gathered}
$$

Take any $v^{*} \in C^{0}\left(\mathcal{U}^{r^{*}}, E^{\prime} \otimes E^{\prime \prime}\right)$ such that $\delta v^{*}=f$. Then $\left(\delta v^{*}-\delta v\right)_{j k}=0$ in $U_{j}^{r_{*}} \cap U_{k}^{r_{*}}$, because $\left(\delta v^{*}\right)_{j k}=f_{j k}$ on the larger set $U_{j}^{r^{*}} \cap U_{k}^{r^{*}}$. Since $\left\{U_{j}^{r_{*}}\right\}$ is a covering of $C$ then $w:=v_{j}-v_{j}^{*}$ is a global section of $E^{\prime} \otimes E^{\prime \prime}$. This shows that $v_{j}$, via $v_{j}^{*}$, extends to a holomorphic section in $U_{j}^{r^{*}}$. In fact, $v_{j}$ is the restriction of $u_{j}=v_{j}^{*}+w$ defined on $U_{j}^{r_{*}}$.

We now derive the bound for $u_{j}$. Suppose that $U_{j}^{r^{*}} \cap U_{k}^{r_{*}}$ is non-empty. By the assumptions, each component of $U_{j}^{r^{*}} \cap U_{k}^{r_{*}}$ intersects $U_{j}^{r_{*}} \cap U_{k}^{r_{*}}$. We have $u_{j}=u_{k}+f_{j k}$ on $U_{j}^{r_{*}} \cap U_{k}^{r_{*}}$ and hence the uniqueness theorem implies that it holds on $U_{j}^{r^{*}} \cap U_{k}^{r_{*}}$ too. And on $U_{j}^{r^{*}} \cap U_{k}^{r_{*}}$, we have $u_{k}=v_{k}$ and $u_{j}=v_{k}-f_{k j}$. We express the identity in coordinates

$$
u_{j}=\tilde{u}_{j} e_{j}, \quad v_{k}=\tilde{v}_{k} e_{k}=\hat{v}_{k j} e_{j}, \quad f_{k j}=\tilde{f}_{k j} e_{k j}=\hat{f}_{k j} e_{j j} .
$$

Let $t_{k j}^{\prime}, t_{k j}^{\prime \prime}$ respectively be the transition matrices of $e_{j}^{\prime}, e_{j}^{\prime \prime}$ for $E^{\prime}, E^{\prime \prime}$. Then $\tilde{t}_{k j}=t_{k j}^{\prime} \otimes t_{k j}^{\prime \prime}$ are the transition matrices of $e_{k j}$ for $E^{\prime} \otimes E^{\prime \prime}$. Then we have

$$
\hat{v}_{k j}=t_{j k}^{\prime} \otimes t_{j k}^{\prime \prime} \tilde{v}_{k}, \quad \hat{f}_{k j}=t_{j k}^{\prime} \otimes I_{d} \tilde{f}_{k j} .
$$

Thus, $\tilde{u}_{j}=\hat{v}_{k j}-\hat{f}_{k j}=t_{j k}^{\prime} \otimes t_{j k}^{\prime \prime} \tilde{v}_{k}-t_{j k}^{\prime} \otimes I_{d} \tilde{f}_{k j}$. We have

$$
\begin{aligned}
\left\|\tilde{u}_{j}\right\|_{L^{2}\left(U_{j}^{r *} \cap U_{k}^{r *}\right)} & =\left\|\tilde{u}_{j} \circ \varphi_{j}^{-1}\right\|_{L^{2}\left(\varphi_{j}\left(U_{j}^{r^{*}} \cap U_{k}^{r_{*}}\right)\right)} \\
& \leq\left\|\left(t_{j k}^{\prime} \otimes t_{j k}^{\prime \prime} \tilde{v}_{k}\right) \circ \varphi_{j}^{-1}\right\|_{L^{2}\left(\varphi_{j}\left(U_{j}^{r^{*}} \cap U_{k}^{r *}\right)\right)}+\left\|\left(t_{j k}^{\prime} \otimes I_{d} \tilde{f}_{k j}\right) \circ \varphi_{j}^{-1}\right\|_{L^{2}\left(\varphi_{j}\left(U_{j}^{r^{*}} \cap U_{k}^{r^{*}}\right)\right)} .
\end{aligned}
$$


Here $t_{j k} \circ \varphi_{j}^{-1}=t_{j k} \circ \varphi_{k}^{-1} \circ \varphi_{k j}$. By the properties of operator norm and $\left\|t_{k j}^{\prime} \otimes t_{k j}^{\prime \prime}\right\|_{D} \leq$ $\left|t_{k j}^{\prime} \otimes t_{k j}^{\prime \prime}\right|_{D} \leq\left|t_{k j}^{\prime}\right|_{D}\left|t_{k j}^{\prime \prime}\right|_{D}$ for $D=\varphi_{j}\left(U_{j}^{r^{*}} \cap U_{k}^{r_{*}}\right)$, we have

$$
\left\|\left(t_{j k}^{\prime} \otimes t_{j k}^{\prime \prime} \tilde{v}_{k}\right) \circ \varphi_{j}^{-1}\right\|_{D}^{2} \leq C_{*}\left|t_{j k}^{\prime}\right|_{D}^{2} \times\left|t_{j k}^{\prime \prime}\right|_{D}^{2} \times\left\|\tilde{v}_{k}\right\|_{\varphi_{k}\left(U_{j}^{r *} \cap U_{k}^{r *}\right)}^{2}
$$

where the constant $C_{*}$ comes from the Jacobian of $z_{k}=\varphi_{k j}\left(z_{j}\right)$. By (A.7), we have

$$
\left\|\tilde{v}_{k} \circ \varphi_{k}^{-1}\right\|_{L^{2}}^{2} \leq K^{2}\|f\|_{L^{2}}^{2}
$$

We also have

$$
\left\|\left(t_{j k}^{\prime} \otimes I_{d} \tilde{f}_{k j}\right) \circ \varphi_{j}^{-1}\right\|_{\varphi_{j}\left(U_{j}^{r *} \cap U_{k}^{r *}\right)} \leq\left|t_{j k}^{\prime} \circ \varphi_{j}^{-1}\right|_{\varphi_{j}\left(U_{j}^{r *} \cap U_{k}^{r *}\right)} \times\|f\|_{\varphi_{j}\left(U_{j}^{r *} \cap U_{k}^{r *}\right)} .
$$

Since $U_{j}^{r^{*}}$ is covered by $\left\{U_{j}^{r^{*}} \cap U_{k}^{r_{*}}\right\}$, we get the desired bound from

$$
\left\|\tilde{u}_{j}\right\|_{L^{2}\left(U_{j}^{r^{*}}\right)} \leq \sum_{k}\left\|\tilde{u}_{j}\right\|_{L^{2}\left(U_{j}^{r^{*}} \cap U_{k}^{r *}\right)}
$$

The argument for the norm $|\cdot|$ is verbatim and we can take the above constant $C_{*}$ to be one.

Corollary A.3. With notations and assumptions in Lemma A.2, the solution $u$ also satisfies

$$
|u|_{\infty, \mathcal{U}^{r^{*}}} \leq C\left(\left|\left\{t_{k j}^{\prime}\right\}\right|_{\mathcal{U}^{r^{*}}}+K\left|\left\{t_{k j}^{\prime}\right\}\right|_{\mathcal{U}^{r^{*}}}\left|\left\{t_{k j}^{\prime \prime}\right\}\right|_{\mathcal{U}^{r^{*}}}\right) \sqrt{\operatorname{rank}\left(E^{\prime} \otimes E^{\prime \prime}\right)}|f|_{\infty, \mathcal{U}^{r^{*}}}
$$

where $C$ does not depend on $E^{\prime}, E^{\prime \prime}$.

The above lemma leads us to the following proposition and definition.

Proposition A.4. Let $\mathcal{U}^{r}=\left\{U_{i}^{r}: i \in \mathcal{I}\right\}$ with $r_{*} \leq r \leq r^{*}$ be a family of nested coverings of a compact complex manifold $C$. Let $E^{\prime}$ (resp. $\left.E^{\prime \prime}\right)$ be a holomorphic vector bundle over $C$ with bases $\left\{e_{j}^{\prime}\right\}$ (resp. $\left.\left\{e_{j}^{\prime \prime}\right\}\right)$ and transition matrices $t_{k j}^{\prime}\left(\right.$ resp. $\left.\left\{t_{k j}^{\prime \prime}\right\}\right)$. Suppose that there is a finite number $K$ such that for any $f \in C^{1}\left(\mathcal{U}^{r^{*}}, E^{\prime} \otimes E^{\prime \prime}\right)$ with $f=0$ in $H^{1}\left(\mathcal{U}^{r^{*}}, E^{\prime} \otimes E^{\prime \prime}\right)$, there is a solution $v \in C^{0}\left(\mathcal{U}^{r_{*}}, E^{\prime} \otimes E^{\prime \prime}\right)$ satisfying $(A .4)$. Then there is a possible different solution $v \in C^{0}\left(\mathcal{U}^{r_{*}}, E^{\prime} \otimes E^{\prime \prime}\right)$ satisfying $(A .4)$ in which $K$ is replaced by

$$
\begin{aligned}
K_{*}\left(E^{\prime} \otimes E^{\prime \prime}\right)=\sup _{u_{1}} \inf _{u_{0}}\left\{\left\|u_{0}\right\|_{\mathcal{U}^{r_{*}}}: \delta u_{0}=\delta u_{1} \text { on } \mathcal{U}^{r_{*}}\right. \\
\left.\left\|\delta u_{1}\right\|_{\mathcal{U}^{r^{*}}}=1, u_{i} \in C^{0}\left(\mathcal{U}^{r_{i}}, E^{\prime} \otimes E^{\prime \prime}\right)\right\} .
\end{aligned}
$$

Proof. By the assumption, $K_{*}=K_{*}\left(E^{\prime} \otimes E^{\prime \prime}\right)$ is well-defined and $K_{*} \leq K$. Fix $u_{1} \in$ $C^{0}\left(\mathcal{U}^{r_{i}}, E^{\prime} \otimes E^{\prime \prime}\right)$. Suppose that $\delta u_{1}=f$ and $\|f\|_{\mathcal{U}^{r^{*}}}=1$. By the definition (A.8), there exists $u_{0}^{j}$ such that $\delta u_{0}^{m}=f$ on $\mathcal{U}^{r_{*}}$ and $\left\|u_{0}^{m}\right\|_{\mathcal{U}^{r *}} \leq K_{*}+1 / m$. By the Cauchy formula on polydiscs, $\left(u_{0}^{m}\right)_{j} \circ \varphi_{j}^{-1}$ is locally bounded in $\varphi_{j}\left(U_{j}\right)$ in sup-norm. We may assume that as $m \rightarrow \infty,\left(u_{0}^{m}\right)_{j}$ converges uniformly to $u_{0}^{\infty}$ on each compact subset of $U_{j}$ for all $j$. This shows that $\left\|\left(u_{0}^{\infty}\right)_{j} \circ \varphi_{j}^{-1}\right\|_{L^{2}(E)} \leq K_{*}$ for any compact subset $E$ of $\varphi_{j}\left(U_{j}\right)$. Since $E$ is arbitrary, we obtain $\left\|u_{0}^{\infty}\right\|_{U^{* *}} \leq K_{*}$. By the uniform convergence, we also have $\delta u_{0}^{\infty}=f$ on $\mathcal{U}^{r_{*}}$. 
Definition A.5. Let $E^{\prime}, E^{\prime \prime}, e_{j}^{\prime}, e_{j}^{\prime \prime}, t_{k j}^{\prime}, t_{k j}^{\prime \prime}$ be as in Proposition $A .4$. Let $t_{k j}^{\prime \prime}\left(S^{m} E^{\prime \prime}\right)$ be the transition matrices of the symmetric power $S^{m} E^{\prime \prime}$ induced by $t_{k j}^{\prime \prime}$. For $m=2,3, \ldots$, we shall call

$$
\begin{aligned}
K\left(E^{\prime} \otimes S^{m} E^{\prime \prime}\right)= & \left|\left\{t_{k j}^{\prime}\left(E^{\prime}\right)\right\}\right|_{\mathcal{U}^{*}} \\
& +K_{*}\left(E^{\prime} \otimes S^{m} E^{\prime \prime}\right)\left|\left\{t_{k j}^{\prime}\left(E^{\prime}\right)\right\}\right|_{\mathcal{U}^{r^{*}}}\left|\left\{t_{k j}^{\prime \prime}\left(S^{m} E^{\prime \prime}\right)\right\}\right|_{\mathcal{U}^{r^{*}}}
\end{aligned}
$$

the generalized small divisors of $E^{\prime} \otimes E^{\prime \prime}$ with respect to $e_{j}^{\prime \prime}, t_{k j}^{\prime \prime}$.

A.3. Donin's smoothing decomposition. Grauert's smoothing decomposition for cochains of analytic sheaves is an important tool. Here we will follow an approach of Donin [8], by specializing for vector bundles.

We first need to introduce coverings by analytic polydiscs.

Lemma A.6. Let $C$ be a compact complex manifold. Let $\left\{U_{i}^{r_{*}}: i \in \mathcal{I}\right\}$ be a finite open covering of $C$, and let $\varphi_{j}$ map $U_{j}^{r}$ biholomorphically onto $\Delta_{r}^{n}$ for $r_{*}<r<r^{*}<1$. Assume further that $\varphi_{i}$ is a biholomorphism defined in a neighborhood of the star $N\left(U_{i}^{r^{*}}\right)$ onto a domain in $\mathbf{C}^{n}$. Suppose that $r_{*}<r_{i}^{\prime}<r_{i}<r^{*}$, and

$$
U_{I}^{r^{\prime}}:=U_{i_{0}}^{r_{0}^{\prime}} \cap \cdots \cap U_{i_{q}}^{r_{q}^{\prime}} \neq \emptyset .
$$

Then for constant $c_{n} \in(0,1)$ depending only on $n$,

$$
\begin{gathered}
\operatorname{dist}\left(\partial\left(\varphi_{i_{q}}\left(U_{I}^{r}\right)\right), \partial\left(\varphi_{i_{q}}\left(U_{I}^{r^{\prime}}\right)\right)\right) \geq c_{n} \kappa \min _{j}\left(r_{j}-r_{j}^{\prime}\right), \\
\kappa:=\inf \left\{1, \frac{\left|\varphi_{i_{q}} \circ \varphi_{i_{\ell}}^{-1}\left(z^{\prime}\right)-\varphi_{i_{q}} \circ \varphi_{i_{\ell}}^{-1}(z)\right|}{\left|z^{\prime}-z\right|}: z, z^{\prime} \in \Delta_{r^{*}}^{n}, \forall U_{i_{0} \ldots i_{q}}^{r^{*}} \neq \emptyset\right\} .
\end{gathered}
$$

Proof. Note that for sets in $\mathbf{C}^{n}$, if $A \subset A^{\prime}, B \subset B^{\prime}$, and $A, B$ are non-empty, then

$$
\operatorname{dist}(A, B) \geq \operatorname{dist}\left(A^{\prime}, B^{\prime}\right) \text {. }
$$

Recall that $\varphi_{i_{q}}$ is a diffeomorphism from a neighborhood $V$ of the star $N\left(U_{i_{q}}\right)$ onto a subset $\hat{V}$ of $\mathbf{C}^{n}$. We have $\partial \varphi_{i_{q}}\left(U_{I}^{r}\right) \subset \cup_{j} \partial \varphi_{i_{q}}\left(U_{i_{j}}^{r}\right)$. Thus

$$
\operatorname{dist}\left(\partial \varphi_{i_{q}}\left(U_{I}^{r}\right), \varphi_{i_{q}}\left(U_{I}^{r^{\prime}}\right)\right) \geq \min _{j} \operatorname{dist}\left(\partial \varphi_{i_{q}}\left(U_{i_{j}}^{r}\right), \varphi_{i_{q}}\left(U_{I}^{r^{\prime}}\right)\right) \geq \min _{j} \operatorname{dist}\left(\partial \varphi_{i_{q}}\left(U_{i_{j}}^{r}\right), \varphi_{i_{q}}\left(U_{i_{j}}^{r^{\prime}}\right)\right) .
$$

We have $\operatorname{dist}\left(\partial\left(\varphi_{i_{q}}\left(U_{i_{j}}^{r}\right), \varphi_{i_{q}}\left(U_{i_{j}}^{r^{\prime}}\right)\right)=\operatorname{dist}\left(\partial\left(\varphi_{i_{q}} \circ \varphi_{i_{j}}^{-1}\left(\Delta_{r}^{n}\right)\right), \varphi_{i_{q}} \circ \varphi_{i_{j}}^{-1}\left(\Delta_{r^{\prime}}^{n}\right)\right)\right.$. Recall that $\varphi_{i_{q}}$ is defined on $N\left(U_{i_{q}}\right) \supset U_{i_{j}}^{r_{*}}$. Then the distance is attained for some $z^{\prime} \in \partial \Delta_{r^{\prime}}^{n}$ and $z \in \partial \Delta_{r}^{n}$. By the definition of $\kappa$, we get the desired estimate.

We will recall the following smoothing decomposition of Donin [8]. Here we restrict to the case of $H^{1}$ and the holomorphic vector bundle to indicate the specific bounds in the estimates.

Theorem A.7 (Donin [8]). Let $C$ be a compact complex manifold and let $\mathcal{U}^{r}\left(r_{*}<r<\right.$ $\left.r^{*}<1\right)$ be a family of open coverings of $C$ as in Lemma A.6. Let $E^{\prime} \otimes E^{\prime \prime}$ be a holomorphic vector bundle of rank $m$ over $C$ and fix a holomorphic base $e_{j}^{\prime}\left(\right.$ resp. $\left.e_{j}^{\prime \prime}\right)$ for $E^{\prime}$ (resp. $\left.E^{\prime \prime}\right)$ over $U_{j}$. Let $r_{*}<r^{\prime \prime}<r^{\prime}<r<r^{*}$, and

$$
r^{\prime}-r^{\prime \prime} \leq r^{*}-r
$$


Assume that

$$
U_{k j}^{r_{*}} \neq \emptyset, \quad \text { whenever } U_{k j}^{r^{*}} \neq \emptyset \text {. }
$$

Let $\left\{f_{j k}\right\} \in Z^{1}\left(\mathcal{U}^{r^{\prime}}, \mathcal{O}\left(E^{\prime} \otimes E^{\prime \prime}\right)\right)$. Then there exist $g \in Z^{1}\left(\mathcal{U}^{r}, \mathcal{O}\left(E^{\prime} \otimes E^{\prime \prime}\right)\right)$ and $u \in$ $C^{0}\left(\mathcal{U}^{r^{\prime \prime}}, \mathcal{O}\left(E^{\prime} \otimes E^{\prime \prime}\right)\right)$ such that

$$
\begin{gathered}
f=g+\delta u, \quad \text { in } C^{1}\left(\mathcal{U}^{r^{\prime \prime}}, \mathcal{O}\left(E^{\prime} \otimes E^{\prime \prime}\right)\right), \\
\|u\|_{\mathcal{U}^{r^{\prime \prime}}}+\|g\|_{\mathcal{U}^{r}} \leq \frac{C_{n}\left|\left\{t_{k j}^{\prime}\right\}\right|\left|\left\{t_{k j}^{\prime \prime}\right\}\right|}{\left(r^{\prime}-r^{\prime \prime}\right) \kappa}\|f\|_{\mathcal{U}^{r^{\prime}}},
\end{gathered}
$$

where $\kappa$ is defined (A.10). The constant $C_{n}$ is independent of $E^{\prime}, E^{\prime \prime}$. Furthermore, $f \mapsto$ $g=L f$ and $f \mapsto u=S f$ are $\mathbf{C}$-linear.

Proof. With $f_{i j}^{r^{\prime}}=f_{i j}$ we are given a cocycle $\left\{f_{i j}^{r^{\prime}}\right\}$ of holomorphic sections of $E^{\prime} \otimes E^{\prime \prime}$ over the covering $\mathcal{U}^{r^{\prime}}$. Recall that $r_{*}<r^{\prime \prime}<r^{\prime}<r<r^{*}$ and $\mathcal{U}^{r^{\prime \prime}}$ is an open covering of $C$.

As in [8], we will apply $L^{2}$-theory for $(0,1)$-forms on a bounded pseudoconvex domain in $\mathbf{C}^{n}$. In our case the domain is actually a polydisc. Fix a holomorphic base $e_{k}^{\prime}=\left(e_{k, 1}^{\prime}, \ldots, e_{k, m}^{\prime}\right)$ for the vector bundle $E^{\prime}$ in $U_{k}^{r^{*}}$ with transition functions $t_{k j}^{\prime}\left(z_{j}\right)$. Analogously, let $t_{k j}^{\prime \prime}\left(z_{j}\right)$ be the transition matrices for basis $e_{k}^{\prime \prime}$ of $E^{\prime \prime}$ for $\mathcal{U}^{r^{*}}$. For brevity, we write $t_{k j}$ for $t_{k j}\left(z_{j}\right)$.

We can write

$$
f_{i j}^{r^{\prime}}=\tilde{f}_{i j}^{r^{\prime}} e_{i j}=t_{k i}^{\prime} \otimes t_{k j}^{\prime \prime} \tilde{f}_{i j}^{r^{\prime}} e_{k k}:=\hat{f}_{i j ; k}^{r^{\prime} ; r^{*}} e_{k k}, \quad \text { on } U_{i}^{r^{\prime}} \cap U_{j}^{r^{\prime}} \cap U_{k}^{r^{*}} .
$$

The $U_{k}^{r^{*}}$ is covered by $\mathcal{U}_{k}^{r^{\prime} ; r^{*}}:=\left\{U_{i}^{r^{\prime}} \cap U_{k}^{r^{*}}\right\}_{i}$, while $\left\{\hat{f}_{i j ; k}^{r^{\prime} ; r^{*}}\right\} \in Z^{1}\left(\mathcal{U}_{k}^{r^{\prime} ; r^{*}}, \mathcal{O}^{m d}\right)$. Now $\left\{\hat{f}_{i j ; k}^{r^{\prime} ; r^{*}} \circ \varphi_{k}^{-1}\right\} \in Z^{1}\left(\varphi_{k}\left(\mathcal{U}_{k}^{r^{\prime} ; r^{*}}\right), \mathcal{O}^{m d}\right)$, where $\varphi_{k}\left(\mathcal{U}_{k}^{r^{\prime} ; r^{*}}\right)$ is a covering of the polydisc $\Delta_{r^{*}}^{n}$. By Lemma A.6, we have

$$
c_{i ; k}:=\operatorname{dist}\left(\partial\left(\varphi_{k}\left(U_{i}^{r^{\prime}} \cap U_{k}^{r^{*}}\right)\right), \varphi_{k}\left(U_{i}^{r^{\prime \prime}} \cap U_{k}^{r}\right)\right) \geq c_{n} \kappa\left(r^{\prime}-r^{\prime \prime}\right) .
$$

Let $d_{i ; k}(z)$ be the distance to $\varphi_{k}\left(U_{i}^{r^{\prime \prime}} \cap U_{k}^{r}\right)$ from $z \in \mathbf{C}^{n}$. Let $\chi$ be a non-negative smooth function in $\mathbf{R}$ so that $\chi(t)=1$ for $t<3 / 4$ and $\chi(t)=0$ for $t>7 / 8$. By smoothing the Lipschitz function $\chi\left(\frac{1}{c_{i ; k}} d_{i ; k}(z)\right)$, we obtain a non-negative smooth function $z \rightarrow \tilde{\phi}_{i ; k}^{r^{\prime \prime} ; r^{\prime}}(z)$ that equals 1 when $d_{i ; k}(z) \leq \frac{1}{2} c_{i ; k}$ and by (A.15) it has compact support in $\varphi_{k}\left(U_{i}^{r^{\prime}} \cap U_{k}^{r^{*}}\right)$. Note that we can achieve

$$
\left|\nabla \tilde{\phi}_{i ; k}^{r^{\prime \prime} ; r^{\prime}}\right|<C_{n} c_{i ; k}^{-1} \leq c_{n} C_{n} \kappa^{-1} /\left(r^{\prime}-r^{\prime \prime}\right) .
$$

Then $\tilde{\phi}_{i ; k}^{r^{\prime \prime} ; r^{\prime}} \circ \varphi_{k}$ is a non negative function with compact support in $U_{i}^{r^{\prime}} \cap U_{k}^{r^{*}}$ such that for $\tilde{\phi}_{k}^{r^{\prime \prime} ; r^{\prime}}:=\sum \tilde{\phi}_{i ; k}^{r^{\prime \prime} ; r^{\prime}}$, we have $\tilde{\phi}_{k}^{r^{\prime \prime} ; r^{\prime}} \circ \varphi_{k}>1 / 2$ in $U_{k}^{r}=\bigcup_{i}\left(U_{i}^{r^{\prime \prime}} \cap U_{k}^{r}\right)$ since $\chi\left(\frac{1}{c_{i ; k}} d_{i ; k}\right)=1$ on $\varphi_{k}\left(U_{i}^{r^{\prime \prime}} \cap U_{k}^{r}\right)$. Then by the mean-value theorem and the first inequality of (A.16), we get

$$
\tilde{\phi}_{k}^{r^{\prime \prime} ; r^{\prime}}\left(\varphi_{k}(x)\right)>1 / 4, \quad \text { if } \operatorname{dist}\left(\varphi_{k}(x), \varphi_{k}\left(U_{k}^{r}\right)\right)<\min _{i} c_{i, k} / C_{*},
$$

for some suitable $C_{*}$. Recall that $c_{n} \leq 1$ and $\kappa_{n} \leq 1$. Since $\operatorname{dist}\left(\varphi_{k}\left(U_{k}^{r}\right), \varphi_{k}\left(\partial U_{k}^{r^{*}}\right)\right)=$ $r^{*}-r^{\prime} \geq c_{n} \kappa\left(r^{\prime}-r^{\prime \prime}\right)$, there is a smooth function $\hat{\phi}_{k}^{r ; r^{*}}: \varphi_{k}\left(U_{k}^{r^{*}}\right) \rightarrow[0,1]$ with compact 
support such that $\hat{\phi}_{k}^{r ; r^{*}}=1$ in $\varphi_{k}\left(U_{k}^{r}\right)$, and

$$
\hat{\phi}_{k}^{r ; r^{*}}(x)<3 / 4, \quad \text { if } \operatorname{dist}\left(\varphi_{k}(x), \varphi_{k}\left(U_{k}^{r}\right)\right)>\min _{i} c_{i, k} / C_{*} \text {. }
$$

Note that the latter can be achieved with

$$
\left|\nabla \hat{\phi}_{k}^{r ; r^{*}}\right|<\tilde{C}_{1} / \min _{i} c_{i, k} \leq C_{2} \kappa^{-1} /\left(r^{\prime}-r^{\prime \prime}\right) .
$$

In $U_{k}^{r^{*}}$, define a non-negative smooth function

$$
\phi_{i ; k}^{r^{\prime \prime} ; r^{\prime}}=\left\{\frac{\tilde{\phi}_{i ; k}^{r^{\prime \prime} ; r^{\prime}}}{1-\hat{\phi}_{k}^{r ; r^{*}}+\tilde{\phi}_{k}^{r^{\prime \prime} ; r^{\prime}}}\right\} \circ \varphi_{k},
$$

where the smoothness follows from the denominator being bigger than $1 / 4$ by (A.17) and (A.18). Thus, $\phi_{i ; k}^{r^{\prime \prime} ; r^{\prime}}$ has compact support in $U_{i}^{r^{\prime}} \cap U_{k}^{r^{*}}$ and $\sum_{i} \phi_{i ; k}^{r^{\prime \prime} ; r^{\prime}}=1$ in $U_{k}^{r}=\bigcup_{i}\left(U_{i}^{r^{\prime \prime}} \cap\right.$ $\left.U_{k}^{r}\right)$, as $\hat{\phi}_{k}^{r ; r^{*}}=1$ on $U_{k}^{r}$. We can verify that

$$
\left|\nabla\left(\phi_{i ; k}^{r^{\prime \prime} ; r^{\prime}} \circ \varphi_{k}^{-1}\right)\right|<C^{\prime} \kappa^{-1} /\left(r^{\prime}-r^{\prime \prime}\right) .
$$

Consider the expression

$$
w_{j ; k}=\sum_{\ell} \phi_{\ell ; k}^{r^{\prime \prime} ; r^{\prime}} \hat{f}_{\ell j ; k}^{r^{\prime} ; r^{*}}
$$

Recall that $\phi_{\ell ; k}^{r^{\prime \prime} ; r^{\prime}}$ has compact support in $U_{\ell}^{r^{\prime}} \cap U_{k}^{r^{*}}$. Thus it is smooth on $\omega:=U_{j}^{r^{\prime}} \cap U_{k}^{r^{*}} \cap U_{\ell}^{r^{\prime}}$ and vanishes on an open set $D$ containing $U_{j}^{r^{\prime}} \cap U_{k}^{r^{*}} \backslash \omega$. On the other hand, $\hat{f}_{\ell j ; k}^{r^{\prime} ; r^{*}}$ is holomorphic in $\omega$. Hence the product $\phi_{\ell ; k}^{r^{\prime \prime} ; r^{\prime}} \hat{f}_{\ell j ; k}^{r^{\prime} ; r^{*}}$ is smooth in $U_{j}^{r^{\prime}} \cap U_{k}^{r^{*}}$. Then $v_{j ; k}=\bar{\partial} w_{j ; k}$ is a smooth $(0,1)$ form in $U_{j}^{r^{\prime}} \cap U_{k}^{r^{*}}$.

Let $\mathcal{A}$ denote the sheaf of smooth functions on $C$. We now pull back the forms from the polydisc $\Delta^{n}$ via $\varphi_{k}$. For each fixed $k$, we have $\left\{w_{j ; k}\right\}_{j} \in C^{0}\left(\mathcal{U}_{k}^{r^{\prime} ; r^{*}}, \mathcal{A}^{m}\right)$. Let us denote $t_{k j}^{\prime} \otimes I$ by $t_{k j}^{\prime}$. By $f_{i j}=f_{i k}-f_{j k}$ and (A.14), we have

$$
t_{k i}^{\prime} \otimes t_{k j}^{\prime \prime} \tilde{f}_{i j}^{r^{\prime}}=t_{k i}^{\prime} \tilde{f}_{i k}^{r^{\prime}}-t_{k j}^{\prime} \tilde{f}_{j k}^{r^{\prime}}
$$

Since $\sum_{i} \phi_{i ; k}^{r^{\prime \prime} ; r^{\prime}}=1=\hat{\phi}_{k}^{r ; r^{*}} \circ \varphi_{k}$ on $U_{k}^{r}$, then by $\delta f=0$ and (A.14), we get on $U_{i}^{r^{\prime}} \cap U_{k}^{r} \cap U_{j}^{r^{\prime}}$

$$
\begin{aligned}
w_{i ; k}-w_{j ; k} & =\sum_{\ell} \phi_{\ell ; k}^{r^{\prime \prime} ; r^{\prime}}\left(\hat{f}_{\ell i ; k}^{r^{\prime} ; r^{*}}-\hat{f}_{\ell j ; k}^{r^{\prime} ; r^{*}}\right)=\sum_{\ell} \phi_{\ell ; k}^{r^{\prime \prime} ; r^{\prime}}\left(t_{k \ell}^{\prime} \otimes t_{k i}^{\prime \prime} \tilde{f}_{\ell i}^{r^{\prime}}-t_{k \ell}^{\prime} \otimes t_{k j}^{\prime \prime} \tilde{f}_{\ell j}^{r^{\prime}}\right) \\
& =\sum_{\ell} \phi_{\ell ; k}^{r^{\prime \prime} ; r^{\prime}}\left(t_{k j}^{\prime} \tilde{f}_{j k}^{r^{\prime}}-t_{k i}^{\prime} \tilde{f}_{i k}^{r^{\prime}}\right)=t_{k j}^{\prime} \tilde{f}_{j k}^{r^{\prime}}-t_{k i}^{\prime} \tilde{f}_{i k}^{r^{\prime}}
\end{aligned}
$$

The latter is holomorphic. Thus $(\delta v)_{i j ; k}=\bar{\partial}(\delta w)_{i j ; k}=0$ on $U_{i}^{r^{\prime}} \cap U_{k}^{r^{*}} \cap U_{j}^{r^{\prime}}$. This shows that

$$
v_{k}:=v_{j ; k}
$$

is actually a $\bar{\partial}$-closed $(0,1)$ form in $U_{k}^{r^{*}}$. Thus $\left(\varphi_{k}^{-1}\right)^{*} v_{k}$ is a $\bar{\partial}$-closed $(0,1)$-form on the polydisk $\Delta_{r^{*}}^{n}$. By the $L^{2}$ theory [20, Thm. 4.4.3] applied to each component of $v_{k}=$ 
$\sum_{\ell=1}^{m} \tilde{v}_{k}^{\ell} e_{k k, \ell}$, we have a bounded linear operator $S: v_{k} \rightarrow u_{k}$ such that $\bar{\partial}\left(\left(\varphi_{k}^{-1}\right)^{*} u_{k}\right)=$ $\left(\varphi_{k}^{-1}\right)^{*} v_{k}$. Returning to the complex manifold via $\varphi_{k}$, we have

$$
\begin{aligned}
\left\|u_{k}\right\|_{U_{k}^{r^{*}}} & =\left\|u_{k} \circ \varphi_{k}^{-1}\right\|_{L^{2}\left(\Delta_{r^{*}}^{n}\right)} \leq C\left\|v_{k} \circ \varphi_{k}^{-1}\right\|_{L^{2}\left(\Delta_{r^{*}}^{n}\right)} \\
& \leq \frac{\tilde{C} \kappa^{-1}\left|\left\{t_{k j}^{\prime}\right\} \|\left\{t_{k j}^{\prime \prime}\right\}\right|}{r^{\prime}-r^{\prime \prime}}\|f\|_{L^{2}\left(\mathcal{U}^{r^{*}}\right)} .
\end{aligned}
$$

Here we have used (A.20), estimate (A.19) and the definition of norm (A.4). Note that the $\tilde{C}$ is independent of the rank since we applied the $L^{2}$ componentwise. Set $\hat{g}_{j ; k}^{r^{\prime} ; r}=w_{j ; k}-u_{k}$ on $U_{j}^{r^{\prime}} \cap U_{k}^{r}$. We obtain

$$
\begin{gathered}
\hat{g}_{i ; k}^{r^{\prime}, r}-\hat{g}_{j ; k}^{r^{\prime}, r}=\hat{f}_{i j ; k}^{r^{\prime} ; *^{*}}, \quad U_{i}^{r^{\prime}} \cap U_{k}^{r} \cap U_{j}^{r^{\prime}}, \\
\max _{j}\left\|\hat{g}_{j ; k}^{r^{\prime} ; r}\right\|_{U_{j}^{r^{\prime}} \cap U_{k}^{r}} \leq \frac{C \kappa^{-1}\left|\left\{t_{k j}^{\prime}\right\} \|\left\{t_{k j}^{\prime \prime}\right\}\right|}{r^{\prime}-r^{\prime \prime}}\|f\|_{\mathcal{U}^{r^{\prime}}} .
\end{gathered}
$$

We have obtained (A.13).

To verify (A.12), we will use the same base $e_{k}$ and take the product of (A.21) with $e_{k}$ in order to obtain on $U_{i}^{r^{\prime \prime}} \cap U_{j}^{r^{\prime \prime}} \cap U_{k}^{r} \cap U_{\ell}^{r}$

$$
g_{i ; k}^{r^{\prime} ; r}-g_{j ; k}^{r^{\prime} ; r}=\hat{f}_{i j ; k}^{r^{\prime} ; r^{*}} e_{k}=f_{i j}^{r^{\prime}}=\hat{f}_{i j ; \ell}^{r^{\prime} ; r^{*}} e_{\ell}=g_{i ; \ell}^{r^{\prime} ; r}-g_{j ; \ell}^{r^{\prime} ; r}
$$

and thus

$$
g_{j ; \ell}^{r^{\prime} ; r}-g_{j ; k}^{r^{\prime} ; r}=g_{i ; \ell}^{r^{\prime} ; r}-g_{i ; k}^{r^{\prime} ; r}, \quad \text { on } U_{i}^{r^{\prime \prime}} \cap U_{j}^{r^{\prime \prime}} \cap U_{k}^{r} \cap U_{\ell}^{r} .
$$

Then we have a (well-defined) holomorphic section

$$
g_{k \ell}^{r}:=g_{i ; \ell}^{r^{\prime} ; r}-g_{i ; k}^{r^{\prime} ; r}, \quad U_{k}^{r} \cap U_{\ell}^{r} .
$$

We verify that $\left\{g_{k \ell}^{r}\right\} \in Z^{1}\left(\mathcal{U}^{r}, \mathcal{O}^{m}\right)$. Set $u_{i}^{r^{\prime \prime}}:=g_{i ; i}^{r^{\prime \prime} ; r}$. Since $r^{\prime} \leq r$ we actually have $\left\{u_{i}^{r^{\prime \prime}}\right\} \in C^{0}\left(\mathcal{U}^{r^{\prime}}, E^{\prime} \otimes E^{\prime \prime}\right)$. However, only on $U_{i}^{r^{\prime \prime}} \cap U_{j}^{r^{\prime \prime}}$, we can verify via (A.23) that

$$
g_{i j}^{r}-f_{i j}^{r^{\prime}}=\left(g_{i ; j}^{r^{\prime \prime} ; r}-g_{j ; j}^{r^{\prime \prime} ; r}\right)-\left(g_{i ; j}^{r^{\prime \prime} ; r}-g_{i ; i}^{r^{\prime \prime} ; r}\right)=u_{i}^{r^{\prime \prime}}-u_{j}^{r^{\prime \prime}} .
$$

The above result is a type of Grauert's smoothing decomposition, which can also be obtained by open mapping theorem. See for instance [14, p. 200]. However, this yields an unknown bound in the estimates.

A.4. Finiteness theorem with bounds. The above smoothing decomposition does not provide a solution to the cohomology equations, i.e. if $f=0$ in $H^{1}\left(\mathcal{U}^{r^{\prime}}, \mathcal{O}\left(E^{\prime} \otimes E^{\prime \prime}\right)\right)$, then there exists $u \in C^{0}\left(\mathcal{U}^{r^{\prime \prime}}, \mathcal{O}\left(E^{\prime} \otimes E^{\prime \prime}\right)\right)$ such that $\delta u=f$ on $\mathcal{U}^{r^{\prime \prime}}$, for some $r^{\prime \prime} \leq r^{\prime}$. We will follow [14] to derive the finiteness theorem with explicit bounds. In particular, this provides solutions of first cohomology equations with bounds on shrinking domains.

We first recall the resolution atlases from [14, p. 194], specializing them for the vector bundles. Assume that we have coordinate charts

$$
\varphi_{k}: U_{k}^{r^{*}} \rightarrow P_{k}:=\varphi_{k}\left(U_{k}^{r^{*}}\right)=\Delta_{n}^{r^{*}}
$$


Define $U_{I}^{r^{*}}=U_{i_{0}}^{r^{*}} \cap \cdots \cap U_{i_{q}}^{r^{*}}$ for $I \in \mathcal{I}^{q+1}$. Then $\varphi_{I}=\left(\varphi_{i_{0}}, \ldots, \varphi_{i_{q}}\right)$ is defined on $U_{I}^{r^{*}}$ with range $\hat{U}_{I}^{r^{*}}$. Unless otherwise stated, we omit the superscript $r^{*}$ in $U_{I}^{r^{*}}$. We can define a proper embedding

$$
\varphi_{I}: U_{I} \rightarrow \hat{U}_{I} \hookrightarrow P_{I}:=\Delta_{n_{q}}^{r^{*}}, \quad n_{q}=n(q+1) .
$$

Then the push-forward of the vector bundle $\left.E^{\prime} \otimes E^{\prime \prime}\right|_{U_{I}}$ defines a coherent analytic sheaf $\left(\varphi_{I}\right)_{*}\left(E^{\prime} \otimes E^{\prime \prime}\right)$ over $P_{I}$ by trivial zero extension; see [14, p. 5, p. 195] and [13, p. 239]. A section $f \in \Gamma\left(U_{I}, E^{\prime} \otimes E^{\prime \prime}\right)$ yields a section $\hat{f}_{I}$ of $\left(\varphi_{I}\right)_{*}\left(E^{\prime} \otimes E^{\prime \prime}\right)$ over $P_{I}$ by

$$
\hat{f}_{I} \circ \varphi_{I}(x)=\left(f_{I}(x), \ldots, f_{I}(x)\right),\left.\quad \hat{f}_{I}\right|_{P_{I} \backslash \hat{U}_{I}}=0 .
$$

Note that $\overline{U^{r^{*}}}$ has a Stein neighborhood. Then following notation in [14, p. 196] we have an epimorphism by Cartan's Theorem A:

$$
\epsilon_{I}:\left.\left.\mathcal{O}^{\ell}\right|_{\Delta_{n_{q}}^{r^{*}}} \rightarrow\left(\varphi_{I}\right)_{*}\left(E^{\prime} \otimes E^{\prime \prime}\right)\right|_{U_{I}}, \quad \ell \geq \operatorname{rank}\left(E^{\prime} \otimes E^{\prime \prime}\right),
$$

where $\epsilon_{I}$ is defined by finitely many global sections defined in a neighborhood of $\overline{P_{I}}$. When $E^{\prime} \otimes E^{\prime \prime}$ is a vector bundle, we take $\ell$ to be the minimal value, the rank of $E^{\prime} \otimes E^{\prime \prime}$, and specify the above $\epsilon_{I}$ by taking

$$
\epsilon_{I}: g_{I} \rightarrow \tilde{g}_{I}:=\left(\varphi_{I}\right)_{*}\left\{g_{I} \circ \varphi_{I} e_{I}\right\} .
$$

Here we want to obtain a more general description without restricting to a vector bundle. Define

$$
C^{q}(\mathcal{U}):=\prod_{I \in \mathcal{I}^{q+1}} \mathcal{O}^{\ell}\left(P_{I}\right)
$$

(Set $\mathcal{O}^{\ell}\left(P_{I}\right)=0$ when $U_{I}^{r^{*}}$ is empty.) We recall that $P_{I}=\Delta_{n_{q}}^{r^{*}}$ is independent of the order of multi-indices. Thus

$$
C^{q}(\mathcal{U}) \cong\left(\mathcal{O}\left(\Delta_{n_{q}}^{r^{*}}\right)\right)^{L}:=\mathcal{O}^{L}\left(\Delta_{n_{q}}^{r^{*}}\right)
$$

Here $L \leq\left|\mathcal{I}^{q+1}\right| \ell$. Let $\mathcal{O}_{h}\left(\Delta_{n_{q}}^{r}\right)$ be the space of holomorphic functions on $\Delta_{n_{q}}^{r}$ with finite $L^{2}$ norm on $\Delta_{n_{q}}^{r}$. Set $P_{I}^{r}=\Delta_{n_{q}}^{r}$ for $I \in \mathcal{I}^{q+1}$. We define a Hilbert space

$$
C_{h}^{q}\left(\mathcal{U}^{r}\right):=\prod_{I \in \mathcal{I}^{q+1}} \mathcal{O}_{h}^{\ell}\left(P_{I}^{r}\right):=\mathcal{O}_{h}^{L}\left(\Delta_{n_{q}}^{r}\right),
$$

which is a subspace of $C^{q}\left(\mathcal{U}^{r}\right)$.

Using the collection $\epsilon=\left\{\epsilon_{I}: I \in \mathcal{I}^{q+1}\right\}$, we define

$$
\mathcal{C}_{h}^{q}\left(\mathcal{U}^{r}, E^{\prime} \otimes E^{\prime \prime}\right):=\epsilon\left(C_{h}^{q}\left(\mathcal{U}^{r}\right)\right) \cong C_{h}^{q}\left(\mathcal{U}^{r}\right) /\left(\operatorname{ker} \epsilon \cap C_{h}^{q}\left(\mathcal{U}^{r}\right)\right),
$$

which is the vector space of $q$-cochains, equipped with the standard coboundary operator $\delta$.

Remark A.8. Our cochains are not necessary alternating. As in [14, p. 35], we let $\mathcal{C}_{a}^{q}\left(\mathcal{U}, E^{\prime} \otimes E^{\prime \prime}\right)$ denotes alternating cochains. For the isomorphism of the two kinds of Cečh cohomology groups; see [14, p. 35] and Serre [37]. Since we are interested in the cohomological solutions with bounds, we fix our nation without requiring that the cochains be alternating. 
Let $\|\cdot\|_{\Delta_{n_{q}}^{r}}$ be the Hilbert space norm on $\mathcal{C}_{h}^{q}\left(\mathcal{U}^{r}\right)$ and set

$$
\|\zeta\|_{\mathcal{U}^{r}}=\inf \left\{\|v\|_{\Delta_{n_{q}}^{r}}: v \in C_{h}^{q}\left(\mathcal{U}^{r}\right), \epsilon(v)=\zeta\right\}, \quad \zeta \in C_{h}^{q}\left(\mathcal{U}^{r}, E^{\prime} \otimes E^{\prime \prime}\right) .
$$

The inclusion $\mathcal{C}_{h}^{q}\left(\mathcal{U}^{r}, E^{\prime} \otimes E^{\prime \prime}\right) \hookrightarrow \mathcal{C}^{q}\left(\mathcal{U}^{r}, E^{\prime} \otimes E^{\prime \prime}\right)$ is continuous and compact ([14, Thm. 3, p. 197]). We also define

$$
\begin{gathered}
Z_{h}^{q}\left(\mathcal{U}^{r}\right):=\epsilon^{-1}\left(Z_{h}^{q}\left(\mathcal{U}^{r}, E^{\prime} \otimes E^{\prime \prime}\right)\right), \\
\|\zeta\|_{\mathcal{U}^{r}}:=\inf \left\{\|v\|_{\Delta_{r}^{n_{q}}}: v \in Z_{h}^{q}\left(\mathcal{U}^{r}\right), \epsilon(v)=\zeta\right\}, \quad \forall \zeta \in Z_{h}^{q}\left(\mathcal{U}^{r}, E^{\prime} \otimes E^{\prime \prime}\right), \\
\bar{v}:=\epsilon(v) .
\end{gathered}
$$

Then $Z_{h}^{q}\left(\mathcal{U}^{r}, E^{\prime} \otimes E^{\prime \prime}\right)$ is an isometric subspace of $\mathcal{C}_{h}^{q}\left(\mathcal{U}^{r}, E^{\prime} \otimes E^{\prime \prime}\right)$ via inclusion. Let $\left\{g_{0}, g_{1}, \ldots\right\}$ be a monotone orthogonal base of $Z_{h}^{1}\left(\mathcal{U}^{r}\right)([14$, p. 141, p. 201]). An important feature of the monotone base is that the vanishing orders of $g_{j}$ at the origin satisfy

$$
\operatorname{ord}_{0} g_{0} \leq \operatorname{ord}_{0} g_{1} \leq \cdots, \quad \lim _{i \rightarrow \infty} \operatorname{ord}_{0} g_{i}=\infty
$$

By [14, Thm. 1, p. 192 and p. 201], for a given $\nu$ there is an $\mu$ such that

$$
g_{i}(Z)=O\left(|Z|^{\nu}\right), \quad i>\mu, \quad Z \in \Delta_{r}^{n_{q}} .
$$

In fact, let the index set be $\mathcal{I}=\{1, \ldots, L\}$. Set $\omega\left(\left(f_{1}, \ldots, f_{L}\right)\right)=\min \left\{(\alpha, Q): f_{\alpha, Q} \neq 0\right\}$ by using order $<$ on $\mathcal{I} \times \mathbf{N}^{m}$ defined by $(\alpha, P)<(\beta, Q)$ if $|P|<|Q|$, or if $|P|=|Q|$ and there is an $\ell$ such that $p_{\ell}<q_{\ell}$ and $p_{\ell^{\prime}}=q_{\ell^{\prime}}$ for all $\ell^{\prime}>\ell$, or if $P=Q$ and $\alpha<\beta$. Then the basis $\left\{g_{j}\right\}$ satisfies

$$
\omega\left(g_{j}\right)<\omega\left(g_{j+1}\right) .
$$

We now return to the case $q=1$ with $n_{q}=2 n$. In the sequel, $\left\{\left|t_{k j}^{\prime}\right|\right\}=\left\{\left|t_{k j}^{\prime}\right|\right\}_{\mathcal{U}^{*}}$ and $\left\{\mid t_{k j}^{\prime \prime}\right\} \mid=\left\{\left|t_{k j}^{\prime \prime}\right|\right\}_{\mathcal{U}^{r^{*}}}$

Theorem A.9 (Donin-Grauert-Remmert). Let $C$ be a compact complex manifold and let $\mathcal{U}^{r}\left(r_{*}<r<r^{*}<1\right)$ be a family of open coverings of $C$ as in Lemma A.6 such that $(A .11)$ holds for all $k, j$. Let $E=E^{\prime} \otimes E^{\prime \prime}$ be a holomorphic vector bundle of positive rank $m$ over $C$ and fix a holomorphic base $e_{j}^{\prime}$ (resp. $e_{j}^{\prime \prime}$ ) for $E^{\prime}$ (resp. $\left.E^{\prime \prime}\right)$ over $U_{j}^{r^{*}}$. Suppose that $r_{*}<r^{\prime \prime}<r^{\prime}<r<r^{*}$ and $r^{\prime}-r^{\prime \prime} \leq r^{*}-r$. Let $\theta=r^{\prime} / r$. Let $\left\{g_{0}, g_{1}, \ldots\right\}$ be a monotone orthogonal base of $Z_{h}^{1}\left(\mathcal{U}^{r}\right)$ as above. Assume that $\mu, \nu$ satisfy $(A .24)$ and

$$
t:=\frac{C_{n} \kappa^{-1}}{\left(r^{\prime}-r^{\prime \prime}\right)\left(r-r^{\prime}\right)^{2 n}} \theta^{\nu}<1 / 2 .
$$

There exist $\overline{g_{m_{0}}}, \ldots, \overline{g_{m_{\mu^{*}}}}$ such that their equivalence classes in $H^{1}\left(\mathcal{U}^{r}, E\right)$ form a $\mathbf{C}$-linear basis of subspace spanned by $\overline{g_{0}}, \cdots, \overline{g_{\mu}}$ in $H^{1}\left(\mathcal{U}^{r}, E\right)$. For any $f \in Z_{h}^{1}\left(\mathcal{U}^{r^{\prime}}, E\right)$ there exists $v \in C_{h}^{0}\left(\mathcal{U}^{r^{\prime \prime}}, E\right)$ satisfying $f=\delta v+\sum_{0}^{\mu^{*}} c_{i} \overline{g_{m_{i}}}$ with

$$
\begin{gathered}
\left|c_{i}\right| \leq \frac{C_{n} \kappa^{-1} A_{r}(E)}{r-r^{\prime}}\|f\|_{\mathcal{U}^{r^{\prime}}}, \\
\|v\|_{\mathcal{U}^{r^{\prime \prime}}} \leq \frac{C_{n} \kappa^{-1} B_{r_{-}}(E)}{r-r^{\prime}}\|f\|_{\mathcal{U}^{r^{\prime}}}, \quad \forall r_{-} \in\left[r^{\prime}, r\right),
\end{gathered}
$$




$$
\begin{gathered}
g_{j}=\sum_{i=0}^{\mu^{*}} c_{j i} \overline{g_{m_{i}}}+\delta \eta_{j}^{*}, \quad \eta_{j}^{*} \in C^{0}\left(\mathcal{U}^{r}, E\right), \\
A_{r}(E)=\left|\left\{t_{k j}^{\prime}\right\}\right|\left|\left\{t_{k j}^{\prime \prime}\right\}\right| \max _{0 \leq i \leq \mu_{*}} \sum_{j=0}^{\mu}\left|c_{j i}\right|, \quad B_{r_{-}}(E)=\left|\left\{t_{k j}^{\prime}\right\}\right|\left|\left\{t_{k j}^{\prime \prime}\right\}\right| \sum_{j=0}^{\mu}\left\|\left\{\eta_{j}^{*}\right\}\right\|_{\mathcal{U}^{r-}} .
\end{gathered}
$$

Furthermore, all $c_{j}=0$ when $f=0$ in $H^{1}(C, E)$.

Remark A.10. The solution operator $f \rightarrow v$ may not be linear. See a proof by Donin [8] to get a linear solution operator for which the constant $C_{*}$ results from a lemma of Schwartz.

Remark A.11. The previous theorem gives a solution $v$, defined on a smaller domain, to the equation $f=\delta v$ (i.e cohomological equations) whenever $f$ is 0 in the first cohomology group. It also provides a bound of the solution in terms of the data. We emphasize that this bound depends on the bundle $E^{\prime} \otimes E^{\prime \prime}$. In the applications we have in mind, we will have to consider a sequence of bundles $\left\{S^{m} E^{\prime \prime}\right\}_{m}$, and we will need to control the growth of these bounds as $m$ goes to infinite, similarly to the small divisors appearing in local dynamical systems.

Proof. Recall that $q=1$ and $n_{1}=2 n$. We may assume that $\left\|g_{j}\right\|_{\Delta_{2 n}^{r}}=1$. By the definition of $\mu, \nu$ and the monotone basis, we have for any $v \in Z_{h}^{1}\left(\mathcal{U}^{r}\right)$,

$$
\left\|v-\sum_{j=0}^{\mu}\left(v, g_{j}\right) g_{j}\right\|_{\Delta_{2 n}^{r^{\prime}}} \leq \frac{C_{n}}{\left(r-r^{\prime}\right)^{2 n}}\left(r^{\prime} / r\right)^{\nu}\|v\|_{\Delta_{2 n}^{r}}
$$

where $C_{n}\left(r-r^{\prime}\right)^{-2 n}$ is the constant $M$ in [14, Thm. 6, p. 191].

Replacing the smoothing lemma in [14, p. 200] by Theorem A.7, we derive some estimates following the proof of the finiteness lemma in [14, p. 201]. By assumption, we have

$$
t=\frac{C_{n} \kappa^{-1}}{\left(r^{\prime}-r^{\prime \prime}\right)\left(r-r^{\prime}\right)^{2 n}} \theta^{\nu}<1 / 2, \quad \theta=\frac{r^{\prime}}{r}<1 .
$$

Let $\zeta_{0}:=f \in Z_{h}^{1}\left(\mathcal{U}^{r^{\prime}}, E^{\prime} \otimes E^{\prime \prime}\right)$. By Theorem A.7, we have for some $\xi_{0} \in Z_{h}^{1}\left(\mathcal{U}^{r}, E^{\prime} \otimes E^{\prime \prime}\right)$

$$
\begin{gathered}
\zeta_{0}=\xi_{0}+\delta \eta_{0}, \\
\left\|\xi_{0}\right\|_{\mathcal{U}^{r}} \leq t^{\prime}\left\|\zeta_{0}\right\|_{\mathcal{U}^{r^{\prime}}}, \quad\left\|\eta_{0}\right\|_{\mathcal{U}^{r^{\prime \prime}}} \leq t^{\prime}\left\|\zeta_{0}\right\|_{\mathcal{U}^{r}},
\end{gathered}
$$

with $t^{\prime}:=\frac{C_{n}\left|\left\{t_{k j}^{\prime}\right\}\right|\left|\left\{t_{k j}^{\prime \prime}\right\}\right|}{\kappa\left(r^{\prime}-r^{\prime \prime}\right)}$. Let $\bar{v}$ denote $\epsilon(v)$. Then $\xi_{0}=\bar{v}_{0}$ for some $v_{0}$ satisfying $\left\|v_{0}\right\|_{\Delta_{2 n}^{r}}=$ $\left\|\xi_{0}\right\|_{\mathcal{U}^{r}} ;$ see $[14$, p. 198]. Consider

$$
w_{1}=v_{0}-\sum_{j=0}^{\mu}\left(v_{0}, g_{j}\right)_{\Delta_{2 n}^{r}} g_{j}, \quad \zeta_{1}=\bar{w}_{1} .
$$

According to (A.30), we have

$$
\left\|\zeta_{1}\right\|_{\mathcal{U}^{r^{\prime}}} \leq\left\|w_{1}\right\|_{\mathcal{U}^{r^{\prime}}} \leq \frac{C_{n}}{\left(r-r^{\prime}\right)^{2 n}}\left(r^{\prime} / r\right)^{\nu}\left\|v_{0}\right\|_{\Delta_{2 n}^{r}} \leq t\left\|\zeta_{0}\right\|_{\mathcal{U}^{r^{\prime}}}
$$


Therefore

$$
\zeta_{0}=\sum_{j=0}^{\mu}\left(v_{0}, g_{j}\right)_{\Delta_{2 n}^{r}} \bar{g}_{j}+\delta \eta_{0}+\zeta_{1}
$$

In general, we have

$$
\begin{gathered}
\zeta_{\ell}=\sum_{j=0}^{\mu}\left(v_{\ell}, g_{j}\right)_{\Delta_{2 n}^{r}} \bar{g}_{j}+\delta \eta_{\ell}+\zeta_{\ell+1}, \\
\left\|v_{\ell}\right\|_{\Delta_{2 n}^{r}}=\left\|\zeta_{\ell}\right\|_{\mathcal{U}^{r}} \leq t^{\prime} t^{\ell}\left\|\zeta_{0}\right\|_{\mathcal{U}^{r^{\prime}}}, \\
\left\|\zeta_{\ell+1}\right\|_{\mathcal{U}^{r^{\prime}}} \leq t\left\|\zeta_{\ell}\right\|_{\mathcal{U}^{r^{\prime}}} \leq t^{\ell+1}\left\|\zeta_{0}\right\|_{\mathcal{U}^{r^{\prime}}} \\
\left\|\eta_{\ell}\right\|_{\mathcal{U}^{r^{\prime \prime}}} \leq t^{\prime} t^{\ell}\left\|\zeta_{0}\right\|_{\mathcal{U}^{r^{\prime}}}
\end{gathered}
$$

Then we have

$$
\begin{gathered}
f=\zeta_{0}=\sum_{j=0}^{\mu} \sum_{\ell=0}^{\infty}\left(v_{\ell}, g_{j}\right)_{\Delta_{2 n}^{r}} \bar{g}_{j}+\delta \sum_{\ell=0}^{\infty} \eta_{\ell}, \\
\sum_{\ell=0}^{\infty}\left|\left(v_{\ell}, g_{j}\right)\right| \leq \sum_{\ell=0}^{\infty}\left\|v_{\ell}\right\|_{\Delta_{2 n}^{r}} \leq \frac{t^{\prime}}{1-t}\left\|\zeta_{0}\right\|_{\mathcal{U}^{r^{\prime}}}, \\
\sum_{\ell=0}^{\infty}\left\|\eta_{\ell}\right\|_{\mathcal{U}^{r^{\prime \prime}}} \leq \frac{t^{\prime}}{1-t}\left\|\zeta_{0}\right\|_{\mathcal{U}^{r^{\prime}}} .
\end{gathered}
$$

So far we have followed the proof of the finiteness lemma in [14, p. 201]. We now finish the proof of the theorem. Let us first find the linearly independent elements $\overline{g_{i_{0}}}, \ldots, \overline{g_{i_{\mu_{*}}}}$. Assume first that all $\overline{g_{i}}=0$ in $H^{1}:=H^{1}\left(\mathcal{U}^{r}, E^{\prime} \otimes E^{\prime \prime}\right)$. Then $\delta \eta_{j}=\overline{g_{j}}$ with $\eta_{j} \in C^{0}\left(\mathcal{U}^{r}, E\right)$. Assume now that $\overline{g_{m_{0}}} \neq 0$ in $H^{1}$ for some $m_{0}$. Then we have two cases again: either $\overline{g_{i}}=c_{i 0} \overline{g_{m_{0}}}+\delta \eta_{i}$ on $\mathcal{U}^{r}$ for all $i \in\{0, \ldots, \mu\} \backslash m_{0}$, or it fails for some $m_{1}$. We repeat this to exhaust all elements so that

$$
\overline{g_{j}}=\delta \eta_{j}^{*}+\sum_{i=0}^{\mu_{*}} c_{j i} \overline{g_{m_{i}}}, \quad \eta_{j}^{*} \in \mathcal{C}^{0}\left(\mathcal{U}^{r}, E\right), \quad 0 \leq j \leq \mu
$$

while $\overline{g_{m_{0}}}, \ldots, \overline{g_{m_{*}}}$ are linearly independent in $H^{1}$. (Note that the above expression means the trivial identity $\overline{g_{j}}=\overline{g_{j}}$ when $j$ is not in $\left\{m_{0}, \ldots, m_{\mu^{*}}\right\}$.) We have obtained (A.28) with the decomposition

$$
\begin{gathered}
f=\sum_{j=0}^{\mu^{*}} c_{j} \overline{g_{m_{j}}}+\delta v \\
c_{j}=\sum_{\ell=0}^{\infty}\left(v_{\ell}, g_{j}\right)_{\Delta_{2 n}^{r}}+\sum_{i=0}^{\mu} c_{i j} \sum_{\ell=0}^{\infty}\left(v_{\ell}, g_{i}\right)_{\Delta_{2 n}^{r}}, \\
v=\sum_{i=0}^{\mu} \sum_{\ell=0}^{\infty}\left(v_{\ell}, g_{i}\right)_{\Delta_{2 n}^{r}} \eta_{i}^{*}+\sum_{\ell=0}^{\infty} \eta_{\ell} .
\end{gathered}
$$


The solution $\eta_{j}^{*}$ in $(\mathrm{A} .31)$ can be bounded in $U^{r_{-}}$for any $r_{-}<r$. Of course we need to estimate $\eta_{j}^{*}$ on $\mathcal{U}^{r^{\prime}}$. Thus, $r_{-} \geq r^{\prime}$. We have

$$
\begin{gathered}
\sum_{j=0}^{\mu} \sum_{\ell=0}^{\infty}\left|\left(v_{\ell}, g_{j}\right)_{\Delta_{2 n}^{r}} c_{j i}\right| \leq \frac{t^{\prime}}{1-t} \sum_{j=0}^{\mu}\left|c_{j i}\right|\left\|\zeta_{0}\right\|_{\mathcal{U}^{r^{\prime}}}, \\
\left\|\left\{\sum_{\ell=0}^{\infty} \eta_{\ell}+\sum_{\ell=0}^{\infty} \sum_{j=1}^{\mu}\left(v_{\ell}, g_{j}\right)_{\Delta_{2 n}^{r}} \eta_{j}^{*}\right\}\right\|_{\mathcal{U}^{r-}} \leq \frac{t^{\prime}}{1-t}\left\{1+\sum_{j=0}^{\mu}\left\|\eta_{j}^{*}\right\|_{\mathcal{U}^{r}-}\right\}\left\|\zeta_{0}\right\|_{\mathcal{U}^{r^{\prime}}} .
\end{gathered}
$$

Set $A_{r}(E)=\left|\left\{t_{k j}^{\prime}\right\}\right|\left|\left\{t_{k j}^{\prime \prime}\right\}\right| \max _{i=0}^{\mu^{*}} \sum_{j=0}^{\mu}\left|c_{j i}\right|$ and $B_{r_{-}}(E)=\left|\left\{t_{k j}^{\prime}\right\}\right|\left|\left\{t_{k j}^{\prime \prime}\right\}\right|\left(1+\sum_{j=0}^{\mu}\left\|\eta_{j}^{*}\right\|_{\mathcal{U}^{r_{-}}}\right)$. We have obtained the required estimates.

Finally, let us assume that $f=0$ in $H^{1}(C, E)$ in order to show that all $c_{j}=0$ and thus $f=\delta v$. Since each $U^{r^{\prime \prime}}$ is Stein, we also have $f=0$ in $H^{1}\left(\mathcal{U}^{r}, E\right)$. Thus $f=\delta \tilde{v}$ with $\tilde{v} \in \mathcal{C}^{0}\left(U^{r^{\prime \prime}}, E\right)$. We get $\delta(\tilde{v}-v)=\sum_{j=0}^{\mu^{*}} c_{j} \overline{g_{m_{j}}}$. By the linear independence, we conclude that $c_{j}=0$. We are done.

Theorem A.12. Let $C$ be a compact complex manifold and let $\mathcal{U}^{r}\left(r_{*} \leq r \leq r^{*}<1\right)$ be nested coverings of $C$ as in Proposition A.19. Let $\mu, \nu, r, r^{\prime}, r^{\prime \prime}, r_{*}, r^{*}$ be given in Theorem A.9, which satisfy (A.25). Let $f \in Z^{1}\left(\mathcal{U}^{r^{\prime}}, E^{\prime} \otimes E^{\prime \prime}\right)$. Suppose that $f=0$ in $H^{1}\left(C, E^{\prime} \otimes E^{\prime \prime}\right)$. Then there exists a solution $\left\{u_{j}\right\} \in C^{0}\left(\mathcal{U}^{r^{\prime}}, E^{\prime} \otimes E^{\prime \prime}\right)$ such that $\delta u=f$ and

$$
\begin{gathered}
\|u\|_{\mathcal{U}^{r^{\prime}}} \leq K\left(E^{\prime} \otimes E^{\prime \prime}\right)\|f\|_{\mathcal{U}^{r^{\prime}}} \\
K\left(E^{\prime} \otimes E^{\prime \prime}\right):=C\left(\left|\left\{t_{k j}^{\prime}\right\}\right|_{\mathcal{U}^{r^{\prime}}}+K_{*}\left(E^{\prime} \otimes E^{\prime \prime}\right)\left|\left\{t_{k j}^{\prime}\right\}\right|_{\mathcal{U}^{r^{\prime}}}\left|\left\{t_{k j}^{\prime \prime}\right\}\right|_{\mathcal{U}^{r^{\prime}}}\right),
\end{gathered}
$$

where $K_{*}\left(E^{\prime} \otimes E^{\prime \prime}\right)$, defined by $($ A.8), satisfies

$$
K_{*}\left(E^{\prime} \otimes E^{\prime \prime}\right) \leq \frac{C_{n} B_{r_{-}}\left(E^{\prime} \otimes E^{\prime \prime}\right)}{\left(r-r^{\prime}\right) \kappa},
$$

where $\kappa$ and $B_{r_{-}}$are defined by (A.10) and (A.29). The same conclusion holds if both sides are in sup norms $|\cdot|_{\mathcal{U}^{r^{\prime}}}$, when $\left(r-r^{\prime}\right) \kappa$ is replaced by $\left(\left(r-r^{\prime}\right) \kappa\right)^{n}$.

Remark A.13. The main conclusion is that (A.32) holds without shrinking the covering $\left\{U_{i}^{r^{\prime}}\right\}$ on which $f$ is defined. The solution operator $f \mapsto u$ may not be linear. The small divisor conditions are carried by $B_{r_{-}}$which is determined by (A.25) and (A.29), while the bounds in Theorem A.7 as smoothing lemma does not involve small divisors.

Proof. By the Leray theorem, we know that $[f]=0$ in $H^{1}\left(\mathcal{U}^{r^{\prime}}, E\right)$. By Theorem A.9, we have a solution $u \in C^{0}\left(\mathcal{U}^{r^{\prime \prime}}, E\right)$ so that

$$
\begin{gathered}
f_{j k}=(\delta u)_{j k}, \quad U_{j}^{r^{\prime \prime}} \cap U_{k}^{r^{\prime \prime}}, \\
\|u\|_{\mathcal{U}^{r^{\prime \prime}}} \leq K\|f\|_{\mathcal{U}^{r^{\prime}}}
\end{gathered}
$$

Then the conclusion follows from Lemma A.2.

When the super norm is used, we first obtain a solution $u=\left\{u_{k}\right\}$ for $\mathcal{U}^{r^{*}}$ for $r^{*}=$ $\left(r^{\prime \prime}+r^{\prime}\right) / 2$, while $($ A.34) takes the form

$$
\|u\|_{\mathcal{U}^{r^{*}}} \leq K\|f\|_{\mathcal{U}^{r^{\prime}}} \leq\left(\sqrt{\pi} r^{\prime}\right)^{n} K|f|_{\mathcal{U}^{r^{\prime}}}
$$


$\operatorname{By} \operatorname{dist}\left(\varphi_{k}\left(U_{k}^{r^{\prime \prime}}\right), \partial \varphi_{k}\left(U_{k}^{r^{*}}\right)\right)=r^{*}-r^{\prime \prime}$ and power series expansion, we have $|u|_{\mathcal{U}^{r^{\prime \prime}}} \leq\left(\sqrt{\pi}\left(r^{*}-\right.\right.$ $\left.\left.r^{\prime \prime}\right)\right)^{-n}\|u\|_{\mathcal{U}^{* *}}$. Then the conclusion follows from Lemma A.2 again.

A.5. Existence of nested coverings. In this subsection, our main goal is to construct nested coverings by using transversality theorems and analytic polyhedrons. We recall that $C_{n}$ is a $n$-dimensional compact complex manifold. We shall omit to mention its dimension in what follows.

We first deal with the transversality for a piecewise smooth boundary of an analytic polyhedron and we then define the general position property of several analytic polyhedrons.

Definition A.14. (a) Let $M_{j}$ be a $C^{1}$ real hypersurface defined by $r_{j}=0$, where $r_{j}$ is a $C^{1}$ function in an open set $\omega_{j}$ of a complex manifold $C$ and $d r_{j} \neq 0$ on $M_{j}$. We say that $M_{1}, \ldots, M_{N}$ are in the general position, if $d r_{i_{0}} \wedge \cdots \wedge d r_{i_{q}} \neq 0$ at each point of $M_{i_{0}} \cap \cdots \cap M_{i_{q}}$ for any $1 \leq i_{0}<\cdots<i_{q} \leq N$.

(b) Let $\omega$ be a proper open set of a complex manifold $C$ and let $f \in \mathcal{O}^{N}(\omega)$. We say that

$$
Q:=Q_{N}(f, \omega):=\left\{z \in \omega,|| f(z) \mid:=\max \left\{\left|f_{1}(z)\right|, \ldots,\left|f_{N}(z)\right|\right\}<1\right\},
$$

is an analytic $N$-polyhedron in $\omega$ if $Q$ is non-empty and relatively compact in $\omega$, and $Q$ does not contain any compact connected component. We say that $Q$ is generic, if

$$
\left(d\left|f_{i_{1}}\right| \wedge \cdots \wedge d\left|f_{i_{\ell}}\right|\right)(x) \neq 0 \quad \forall x \in\left\{\left|f_{i_{1}}\right|=\cdots=\left|f_{i_{\ell}}\right|=1\right\} \cap \partial Q
$$

for all $i_{1}<\cdots<i_{\ell}$ and $1 \leq \ell \leq N$.

We will apply transversality theorems. This requires us to use open submanifolds in $\mathbf{C}^{n}$ which may not be closed in $\mathbf{C}^{n}$. Since $Q_{N}=Q_{N}(f, \omega)$ does not contain compact connected component, the closure of each connected component of $Q_{N}$ must intersect some $Q_{N}^{i}:=\left\{\left|f_{i}\right|=1\right\} \cap \omega$. We will call $Q_{N}^{i}$ a face of $Q_{N}$. Removing each $Q_{N}^{i}$ from $\omega$ if it does not intersect $\bar{Q}_{N}$, we get a new $\omega$ such that $\bar{Q}_{N}$ intersects each $Q_{N}^{i}$. Applying the same procedure to $Q_{N}^{i_{1} \ldots i_{k}}:=Q_{N}^{i_{1}} \cap \cdots \cap Q_{N}^{i_{k}}$, we may assume that the non-empty intersection of any number of $Q_{N}^{1}, \ldots Q_{N}^{N}$ intersects $\bar{Q}_{N}$. By (A.36), the closed set $\bar{Q}_{N}$ does not intersect the closed subset of $\omega$ defined by

$$
\left(d\left|f_{i_{1}}\right| \wedge \cdots \wedge d\left|f_{i_{\ell}}\right|\right)(x)=0 \quad\left|f_{i_{1}}\right|(x)=\cdots=\left|f_{i_{\ell}}\right|(x)=1 .
$$

Removing the above sets from $\omega$, we find a neighborhood $\omega^{*}$ of $\bar{Q}_{N}$ such that if $Q_{N}^{i_{1} \ldots i_{k}}$ with $i_{1}<i_{2}<\cdots<i_{k}$ intersects $\omega^{*}$, then it intersects $\bar{Q}_{N}$ and it is a codimension $k$ smooth submanifold in $\omega^{*}$. For brevity we will call $\omega^{*}$ a neat neighborhood of $Q$. We will take $\omega=\omega^{*}$ without specifying $\omega^{*}$.

Definition A.15. Let $\omega_{i}$ be open sets in $C$. For $i=0, \ldots, p$, assume that $\phi_{i} \in \mathcal{O}^{N_{i}}\left(\omega_{i}\right)$ and $Q_{N_{i}}\left(\phi_{i}, \omega_{i}\right)$ is an analytic polyhedron in $\omega_{i}$. We say that they are in the general position, if all faces $Q_{N_{i}}^{j}$ for $1 \leq j \leq N_{i}$ and $0 \leq i \leq p$ are in general position. More precisely, $\omega_{N_{i}}^{*} \cap Q_{N_{i}}^{j}$ are in the general position, where each $\omega_{i}^{*}$ is a neat neighborhood of $\overline{Q_{N_{i}}}$. 
Let us describe some elementary properties of generic analytic polyhedrons. If $Q_{N}(f, \omega)$ is defined in $\omega$ by $\left(\right.$ A.35), we denote for $\rho=\left(\rho_{1}, \ldots, \rho_{N}\right)$

$$
Q_{N}^{\rho}(f, \omega):=\left\{z \in \omega:\left|f_{j}(z)\right|<\rho_{j}, j=1, \ldots, N\right\} .
$$

Lemma A.16. Let $Q_{N_{i}}=Q_{N_{i}}\left(\phi_{i}, \omega_{i}\right)$ be generic polyhedrons in $C$ for $0 \leq i \leq p$. Suppose that $Q_{N_{0}}, \cdots, Q_{N_{p}}$ are in the general position. Then

$$
Q_{N_{0}+\cdots+N_{p}}\left(\left(\phi_{0}, \ldots, \phi_{p}\right), \omega_{0} \cap \cdots \cap \omega_{p}\right)=Q_{N_{0}} \cap \cdots \cap Q_{N_{p}},
$$

if non-empty, is a generic $N_{0}+\cdots+N_{p}$ analytic polyhedron in $\omega_{0 \cdots p}:=\omega_{0} \cap \cdots \cap \omega_{p}$.

Proof. Let $N=N_{0}+\cdots+N_{p}$. It is clear that $Q:=Q_{N_{0}} \cap \cdots \cap Q_{N_{p}}=Q_{N}\left(\left(\phi_{0}, \ldots, \phi_{p}\right), \omega_{i_{0} \cdots i_{p}}\right)$. Since $\bar{Q} \subset \cap \overline{Q_{N_{i}}}$, then $\bar{Q}$ is compact in $\omega_{0 \cdots p}$. Write $\left(\phi_{0}, \ldots, \phi_{p}\right)=\left(\psi_{1}, \cdots, \psi_{N}\right)$. Suppose that $x \in \partial Q$. Since $\bar{Q}$ is compact in $\omega$, then there exist $\mu_{1}<\cdots<\mu_{m}$ with $m \geq 1$ such that $\left|\psi_{\mu_{i}}(x)\right|=1$ and $\left|\psi_{j}(x)\right|<1$ for $j \neq \mu_{\ell}$. By the assumption of the general position, we see that the faces of $Q$ are in the general position.

Let $X, Y$ be smooth real manifolds without boundary and $W$ a smooth submanifold of $Y$. Following [10, p. 50], we say that a smooth mapping $h: X \rightarrow Y$ is transversal to $W$ at $x \in X$, denoted by $h$ 币 $W$ at $x$, if either $h(x) \notin W$ or

$$
T_{h(x)} W+d h\left(T_{x} X\right)=T_{h(x)} Y .
$$

Denote $h \mp W$ on $A$ if $h$ 历 $W$ at each $x \in A \subset X$. When $h$ is the inclusion, we denote $h \pitchfork W$ on $A$ by $X \mp W$ on $A$. Finally, extending Definition A.14 $(a)$, we say that smooth real submanifolds $W_{0}, \ldots, W_{k}$ in $Y$ are in the general position if for any $0 \leq i_{1}<\cdots<i_{m} \leq k$ we have

$$
\bigwedge_{\ell=1}^{k} \bigwedge_{j=1}^{d_{i_{\ell}}} d r_{i_{\ell}, j}(y) \neq 0, \quad \forall y \in W_{i_{1}} \cap \cdots \cap W_{i_{m}},
$$

where $W_{i} \subset \omega_{i}$ is defined by $r_{i, 1}=\cdots=r_{i, d_{i}}=0$ with $d r_{i, 1} \wedge \cdots \wedge d r_{i, d_{i}} \neq 0$ at each point of $W_{i}$. Thus $d_{i}$ is the codimension of $W_{i}$ in $\omega_{i}$. It is clear that (A.37) holds if and only if

$$
W_{i_{j}} \pitchfork\left(W_{i_{1}} \cap \cdots \cap W_{i_{j-1}}\right) \text { at } y, \quad \forall y \in W_{i_{1}} \cap \cdots \cap W_{i_{k}}, 0<j \leq m .
$$

For an analytic $N$-polyhedron $Q_{N}$ in $\omega$ with faces $Q_{N}^{1}, \ldots, Q_{N}^{N}$, we call $Q_{N}^{i_{1} \cdots i_{k}}=Q_{N}^{i_{1}} \cap$ $\cdots \cap Q_{N}^{i_{k}}$ with $i_{1}<\cdots<i_{k}$ and $k \geq 1$ an edge of $Q$. When $Q_{N}$ is generic, a nonempty edge $Q_{N}^{i_{1} \cdots i_{k}}$ is a codimension $k$ submanifold in $\omega$. Let $\left\{Q_{N}^{1} \cdots, N_{N}^{N^{\prime}}\right\}$ be the set of all edges, with the first $N$ edges being the faces.

Proposition A.17. Let $Q_{N_{i}}=Q_{N_{i}}\left(\phi_{i}, \omega_{i}\right)$ be generic polyhedrons in $C$ for $0 \leq i \leq p$ with $\omega_{i}$ being a neat neighborhood of $\bar{Q}_{N_{i}}$. Then $Q_{N_{0}}, \ldots, Q_{N_{p}}$ are in the general position if and only if any $0 \leq i_{1}<\cdots<i_{k} \leq p$ and $1 \leq j_{\ell} \leq N_{i_{\ell}}^{\prime}$, the edges $Q_{N_{i_{1}}}^{j_{1}}, \cdots, Q_{N_{i_{k}}}^{j_{k}}$ are in the general position. Equivalently, each edge $Q_{N_{\ell}}^{s}$ intersects transversally with each edge of the intersection of any number of $Q_{N_{0}}, \ldots, Q_{N_{\ell-1}}$, for $\ell=1, \ldots, p$.

Proof. Since each edge of a polyhedron is the intersection of its faces, it is clear that if $Q_{N_{0}}, \ldots, Q_{N_{p}}$ are in the general position, then the edges $Q_{N_{i_{1}}}^{j_{1}}, \cdots, Q_{N_{i_{k}}}^{j_{k}}$ are in the general position for $0 \leq i_{1}<\cdots<i_{k} \leq p$. 
Conversely, let $\phi_{i}=\left(\phi_{i, 1}, \ldots, \phi_{i, N_{i}}\right)$ and let $\psi_{1}, \ldots, \psi_{m}$ be a subset of $\phi_{0,1}, \ldots, \phi_{0, N_{0}}, \ldots$, $\phi_{p, 1}, \ldots, \phi_{p, N_{p}}$. We emphasize that we do not assume that the latter are distinct functions, although $\phi_{i, 1}, \ldots, \phi_{i, N_{i}}$ are distinct by the general position property of the faces of $Q_{N_{i}}$. Suppose that $\psi_{\ell}$ is in $\left\{\phi_{i_{\ell}, 1}, \ldots, \phi_{i_{\ell}, N_{i_{\ell}}}\right\}$. We need to show that

$$
d\left|\psi_{1}\right| \wedge \cdots \wedge d\left|\psi_{m}\right|(x) \neq 0
$$

if for all $\ell,\left|\psi_{\ell}\right|(x)=1$ and $x \in \bar{Q}_{N_{i_{\ell}}}$. Without loss of generality, we may assume that $i_{1} \leq i_{2} \leq \cdots \leq i_{m}$. Thus

$$
\left(\psi_{1}, \ldots, \psi_{m}\right)=\left(\tilde{\psi}_{\alpha_{0}}, \ldots, \tilde{\psi}_{\alpha_{\ell}}\right), \quad \alpha_{1}<\alpha_{2}<\cdots<\alpha_{\ell}
$$

with $\tilde{\psi}_{\alpha_{\beta}}$ being a non-empty subset of components of $\phi_{\alpha_{\beta}}$. Without loss of generality, we may assume that $\tilde{\psi}_{\alpha_{\beta}}=\left(\phi_{\alpha_{\beta}, 1}, \ldots, \phi_{\alpha_{\beta}, \gamma_{\beta}}\right)$ with $\gamma_{\beta}>0$. Thus $\left|\phi_{\alpha_{\beta}, 1}\right|=\cdots=\left|\phi_{\alpha_{\beta}, \gamma_{\beta}}\right|=1$ define an edge $W_{\alpha_{\beta}}$ of $Q_{\alpha_{\beta}}$. Then (A.39) is equivalent to

$$
\left(\bigwedge_{\delta=1}^{\gamma_{\ell}} d\left|\phi_{\alpha_{\ell}, \delta}\right|\right) \wedge\left(\bigwedge_{\ell^{\prime}=1}^{\ell-1} \bigwedge_{\delta=1}^{\gamma_{\ell^{\prime}}} d\left|\phi_{\alpha_{\ell^{\prime}}, \delta}\right|\right)(x) \neq 0 .
$$

The equivalence of (A.37) and (A.38) implies that (A.39) follows from the assumption that $W_{\alpha_{\ell}} \pitchfork\left(W_{\alpha_{1}} \cap \cdots \cap W_{\alpha_{\ell-1}}\right)$, for $\alpha_{1}<\alpha_{2}<\cdots<\alpha_{\ell}$.

Lemma A.18 (Golubitsky-Guillemin [10, p. 53]). Let $X, B$, and $Y$ be smooth manifolds with $W$ a submanifold of $Y$. Let $\psi: B \rightarrow C^{\infty}(X, Y)$ be a mapping (not necessarily continuous) and define $\Psi: X \times B \rightarrow Y$ by $\Psi(x, b)=\psi(b)(x)$. Assume that $\Psi$ is smooth and that $\Psi$ ॠ $W$. Then the set $\{b \in B \mid \psi(b) \pitchfork W\}$ is dense in $B$.

Proposition A.19. Let $C$ be a compact complex manifold of dimension n. Let $\left\{U_{i}: i=\right.$ $1, \ldots, m\}$ be a finite open covering of $C$. Assume that $\varphi_{j}$ is a biholomorphism from a neighborhood $\omega_{j}$ of the star $N\left(U_{j}\right)$ of $U_{j}$ onto $\hat{\omega}_{j} \subset \mathbf{C}^{n}$ such that $U_{j}=\varphi_{j}^{-1}\left(\Delta_{n}\right)=Q_{n}\left(\varphi_{j}, \omega_{j}\right)$. There exists $\delta>0$ satisfying the following:

(a) For each $j$, there are a relatively compact open set $\tilde{\omega}_{j}$ (resp. $\left.\tilde{U}_{j}\right)$ in $\omega_{j}$ (resp. $\left.\tilde{\omega}_{j}\right)$ and a dense open set $A_{j}$ of $\Delta_{n}^{\delta}$ such that if $c_{j} \in A_{j}$, then $\tilde{\varphi}_{j}:=\varphi_{j}-c_{j}$ is a biholomorphic mapping from $\tilde{U}_{j}$ onto $\Delta_{n}$, and $\tilde{U}_{1}:=Q_{n}\left(\tilde{\varphi}_{1}, \tilde{\omega}_{1}\right), \ldots, \tilde{U}_{m}:=$ $Q_{n}\left(\tilde{\varphi}_{m}, \tilde{\omega}_{m}\right)$ are generic n-polyhedrons in the general position, where $\left\{\tilde{U}_{1}, \ldots \tilde{U}_{m}\right\}$ remains an open covering of $C$ and $\tilde{\omega}_{j}$ is a neighborhood of $N\left(\tilde{U}_{j}\right)$. In particular each $\tilde{\varphi}_{j}$, a translation of $\varphi_{j}$, is injective on $\tilde{\omega}_{j}$.

(b) There is $0<r_{*}<1$ such that if $r_{*} \leq \rho_{i} \leq 1$, then $\tilde{U}_{i_{0}}^{\rho_{0}}, \ldots, \tilde{U}_{i_{q}}^{\rho_{q}}$ are generic $n$-polyhedrons in the general position, where $\tilde{U}_{i}^{\rho}:=\tilde{\varphi}_{i}^{-1}\left(\Delta_{n}^{\rho}\right)$.

Proof. (a) We will apply the transversality theorem for real submanifolds in $\mathbf{C}^{n}$. Therefore, we will use old coordinate charts $\varphi_{j}$ to map edges of polyhedrons $Q_{j}\left(\varphi_{j}, \omega_{j}\right)$ into $\mathbf{C}^{n}$. Set $c_{1}=0, \tilde{\varphi}_{1}=\varphi_{1}, \tilde{U}_{1}=U_{1}$. Let $\hat{W}_{1}, \ldots, \hat{W}_{L_{0}}$ be all edges of $\Delta_{n}$. Let $\tilde{U}_{1}^{1}, \ldots, \tilde{U}_{1}^{N^{\prime}}$ be all edges of $\tilde{U}_{1}$. Set $\widetilde{W}_{1}^{\ell}=\varphi_{2}\left(\omega_{2} \cap \tilde{U}_{1}^{\ell}\right)$. Define

$$
\Psi: \mathbf{C}^{n} \times \Delta_{n}^{\delta} \rightarrow Y:=\mathbf{C}^{n}
$$

with $\Psi(x, b)=x+b$ and $\psi^{b}(x)=\Psi(x, b)$. Let $\left.\psi^{b}\right|_{\widehat{W}_{\ell^{\prime}}}$ be the restriction of $\psi^{b}$ to $\hat{W}_{\ell^{\prime}}$. Applying Lemma A.18, mainly the density assertion in the lemma, finitely many times in 
which $W=\widetilde{W_{1}^{\ell}}$, we can find $b_{2} \in \Delta_{n}^{\delta}$ such that

$$
\left.\psi^{b_{2}}\right|_{\widehat{W}_{\ell^{\prime}}} \pitchfork \widetilde{W_{1}^{\ell}} \quad \text { on } \varphi_{2}\left(\overline{\tilde{U}_{1}} \cap \overline{\omega_{2}^{\prime}}\right), \quad \forall \ell, \ell^{\prime}
$$

where $\omega_{2}^{\prime}$ is a relatively compact open subset of $\omega_{2}$ which is independent of $\delta$, and $\overline{U_{2}} \subset \omega_{2}^{\prime}$. We also remark that $(\mathrm{A} .18)$ can be applied for finitely many times since $\varphi_{2}\left(\overline{\tilde{U}_{1}} \cap \overline{\omega_{2}^{\prime}}\right)$ is compact. Since $\overline{\tilde{U}_{1}} \cap \overline{U_{2}}$ is compact, then

$$
\left.\psi^{c_{2}}\right|_{\widehat{W}_{\ell^{\prime}}} \text { 帀 } \widetilde{W}_{1}^{\ell} \quad \text { on } \varphi_{2}\left(\overline{\tilde{U}_{1}} \cap \overline{\omega_{2}^{\prime}}\right), \quad \forall \ell, \ell^{\prime}
$$

when $\left|c_{2}-b_{2}\right|$ is sufficiently small. Applying $\varphi_{2}^{-1}$ to (A.40) yields

$$
\varphi_{2}^{-1}\left(\left.\psi^{c_{2}}\right|_{\widehat{W}_{\ell^{\prime}}}\right) \pitchfork\left(\omega_{2} \cap \tilde{U}_{1}^{\ell}\right) \quad \text { on } \overline{\tilde{U}_{1}} \cap \overline{\omega_{2}^{\prime}}, \quad \forall \ell, \ell^{\prime} .
$$

With $c_{2}$ being determined, set

$$
\tilde{\varphi}_{2}^{-1}=\varphi_{2}^{-1}\left(\mathrm{I}+c_{2}\right)
$$

Thus $\tilde{\varphi}_{2}=\varphi_{2}-c_{2}$. When $\delta$ and $\left|c_{2}-b_{2}\right|$ are sufficiently small, we have $\overline{\tilde{U}_{2}}=\tilde{\varphi}_{2}^{-1}\left(\overline{\Delta_{n}}\right) \subset$ $\omega_{2}^{\prime}$. Therefore, (A.41) implies that every edge of $\tilde{U}_{2}$ intersects each edge of $\tilde{U}_{1}$. We have determined $\tilde{U}_{2}=\tilde{\varphi}_{2}^{-1}\left(\Delta_{n}\right)$.

We have verified $(a)$ when $m=2$. Let us assume that it also holds for $m \geq j$. By Lemma A.16, each edge of a non-empty intersection of any number of $\tilde{U}_{1}, \ldots, \tilde{U}_{j}$ is a smooth submanifold. We remark the above transversality argument mainly uses the fact that $\varphi_{2}$ is a biholomorphism, while each edge of $\tilde{U}_{1}$ is a smooth submanifold.

To repeat the above argument for $m=2$ in details, we list all edges of all possible intersections of $\tilde{U}_{1}, \ldots, \tilde{U}_{j}$ as $W_{1}^{\prime}, \ldots, W_{L}^{\prime}$ so that each $W_{j}$ is an edge of some analytic polyhedron $U_{j}^{\prime}$, where $U_{j}^{\prime}$ is the intersection of some of $\tilde{U}_{1}, \ldots, \tilde{U}_{j^{\prime}}$ which are in general position by the induction hypothesis as mentioned above. Therefore, by Lemma A.16, each $U_{\ell}^{\prime}$ is generic. Now we are in the situation of $m=2$ by considering the sets of two analytic polyhedrons $\left\{U_{\ell}^{\prime}, U_{j+1}\right\}$ one by one for $\ell=1, \ldots, j^{\prime}$. Here $U_{j+1}=\varphi_{j+1}^{-1}\left(\Delta_{n}\right)$ with $\varphi_{j+1}$ being biholomorphic in a neighborhood of $N\left(U_{j+1}\right)$. Therefore, we can find $\tilde{\varphi}_{j+1}=\varphi_{j+1}-c_{j+1}$ such that each edge of $\tilde{U}_{j+1}$ intersects each $W_{\ell}^{\prime}$ transversally on $\overline{\tilde{U}_{j+1}} \cap \overline{U_{\ell}^{\prime}}$.

The above argument shows the existence of $c_{1}, \ldots, c_{N}$ in $\Delta_{n}^{\delta}$ when $\delta$ is sufficiently small. The openness property on $A_{j}$ is clear, since by shrinking $\tilde{\omega}_{j}$ slightly the general position and generic properties are preserved under small perturbation of $c_{j}$. Then density of $A_{j}$ when $\delta$ is sufficiently small can also be achieved; indeed when $c_{j}$ is sufficiently small, we may shrink $\omega_{j}$ slightly and apply the above argument by replacing $\varphi_{j}-c_{j}$ with $\varphi_{j}$. Finally, $\left\{\tilde{U}_{1}, \ldots, \tilde{U}_{N}\right\}$ still covers $C$ when $\delta$ is sufficiently small. We have verified $(a)$.

The assertion $(b)$ follows from $(a)$ and Proposition A.17. Indeed, we first note that when $r_{*}$ is less than 1 , but it is sufficiently close to 1 , the $\partial Q^{\rho}\left(\tilde{\varphi}_{j}\right)$ is in a given neighborhood of $\partial Q\left(\tilde{\varphi}_{j}, \tilde{\omega}_{j}\right)$, as $Q^{\rho}\left(\tilde{\varphi}_{j}, \tilde{\omega}_{j}\right)$ does not have any compact connected component. By the relative compactness of $Q_{n}\left(\tilde{\varphi}_{i}, \tilde{\omega}_{i}\right)$, the condition (A.36) with $f_{j}$ being replaced by $f_{j} / \rho_{j}$ and the general position condition remain true when $\rho_{j}$ are in $\left[r_{*}, 1\right]$ when $r_{*}<1$ is sufficiently close to 1 . The proof is complete.

The following is a basic property of a generic analytic polyhedron. 
Proposition A.20. Let $C$ be a compact complex manifold of dimension n. Let $Q_{N}(f, \omega)$ be a generic analytic $N$-polyhedron $C$ defined by $(A .35)$ and $(A .36)$. There exists $r_{*} \in(0,1)$ satisfying the following.

(a) If $\rho=\left(\rho_{1}, \ldots, \rho_{N}\right)$ and $\rho^{\prime}=\left(\rho_{1}^{\prime}, \ldots, \rho_{N}^{\prime}\right)$ satisfy $r_{*} \leq \rho_{i}^{\prime} \leq \rho_{i} \leq 1$, every connected component of $Q_{N}^{\rho}(f, \omega)$ intersects $Q_{N}^{\rho^{\prime}}(f, \omega)$ and the latter is non-empty.

(b) There are finitely many open sets $\omega_{j}^{\prime \prime}$ in $C$ and smooth diffeomorphisms $\phi_{j}$ sending $\omega_{j}^{\prime \prime}$ onto $\hat{\omega}_{j}^{\prime \prime}$ in $\mathbf{R}^{2 n}$ such that $\left\{\omega_{j}^{\prime \prime}\right\}$ covers $\partial Q_{N}(f, \omega)$, and for any $p_{0}, p_{1} \in \phi_{j}\left(\omega_{j}^{\prime \prime} \cap\right.$ $\left.Q_{N}^{\rho}(f, \omega)\right)$ there is a smooth curve $\gamma$ in $\phi_{j}\left(\omega_{j}^{\prime \prime} \cap Q_{N}^{\rho}(f, \omega)\right)$ connecting $p_{0}$ and $p_{1}$ with length $|\gamma| \leq C\left|p_{1}-p_{0}\right|$, where $C$ depends only on $\phi_{j}$ and $\omega_{j}^{\prime \prime}$.

Proof. (a) Set $Q=Q_{N}(f, \omega)$ and $Q^{\rho}=Q_{N}^{\rho}(f, \omega)$. For each $x \in \partial Q$, we find $\mu_{1}<\cdots<\mu_{m}$ with $m \leq N$ such that

$$
\left|f_{\mu_{i}}(x)\right|=1, \quad i \leq m ; \quad\left|f_{j}(x)\right|<1, \quad j \neq \mu_{1}, \ldots, \mu_{m} .
$$

Note that $\left\{\mu_{1}, \ldots, \mu_{m}\right\}$ is uniquely determined by $x$. By the transversality condition (A.36), we have $m \leq 2 n$. Choose an open set $\omega^{\prime}$ such that $x \in \omega^{\prime} \subset \omega$ and

$$
\left|f_{i}(z)\right|<1, \quad \forall z \in \overline{\omega^{\prime}}, i \neq \mu_{1}, \ldots, \mu_{m} .
$$

In particular, we have

$$
Q \cap \omega^{\prime}=\left\{z \in \omega^{\prime}:\left|f_{\mu_{i}}(z)\right|<1, \quad i=1, \ldots, m\right\} .
$$

By (A.36), we can take $\left(\left|f_{\mu_{1}}\right|, \ldots,\left|f_{\mu_{m}}\right|\right)$ to be the first $m$ components of a smooth diffeomorphism $\varphi: \omega^{\prime} \rightarrow \hat{\omega}$, shrinking $\omega^{\prime}$ if necessary. Taking a smaller open subset $\omega^{\prime \prime}$ of $\omega^{\prime}$ with $x \in \omega^{\prime \prime}$, we may assume that

$$
t \zeta \in \hat{\omega}, \quad \forall \zeta \in \hat{\omega}^{\prime \prime}:=\varphi\left(\omega^{\prime \prime}\right), \quad 1-\delta \leq t \leq 1,
$$

for some $\delta \in(0,1]$.

Since $\partial Q$ is compact, there exists $\left\{x_{j}, \omega_{j}^{\prime \prime}, \omega_{j}^{\prime}: j=1, \ldots, k\right\}$ satisfying the following:

(a) The $k$ is finite. For each $j$, we have that $x_{j} \in \omega_{j}^{\prime \prime} \subset \omega_{j}^{\prime} \subset \omega, x_{j} \in \partial Q$, and $\omega_{j}^{\prime}$ is an open subset of $\omega$. For each $j$, we have $m_{j}$ and $\mu_{j, 1}<\ldots<\mu_{j, m_{j}}$, which are the numbers associated to $x_{j}$, so that (A.42) holds for $x=x_{j} .\left\{\omega_{1}^{\prime \prime}, \ldots \omega_{k}^{\prime \prime}\right\}$ is an open covering of $\partial Q$.

(b) $\left|f_{\mu_{j, \ell}}\left(x_{j}\right)\right|=1$ for $\ell=1, \ldots, m_{j}$ and

$$
\begin{gathered}
M_{j}:=\sup _{z \in \overline{\omega_{j}^{\prime}}}\left\{\left|f_{i}(z)\right|: i \neq \mu_{j, 1}, \ldots, \mu_{j, m_{j}}\right\}<1, \\
\omega_{j}^{\prime} \cap Q=\left\{z \in \omega_{j}^{\prime}:\left|f_{\mu_{j, \ell}}(z)\right|<1, \ell=1, \ldots, m_{j}\right\} .
\end{gathered}
$$

Here we set $M_{j}=0$ if $m_{j}=N$.

(c) The $\left(\left|f_{\mu_{j, 1}}\right|, \ldots,\left|f_{\mu_{j, m_{j}}}\right|\right)$ are the first $m_{j}$ components of a smooth diffeomorphism $\phi_{j}$ from $\omega_{j}$ onto a subset $\hat{\omega}_{j}$ of $\mathbf{C}^{n}$. There exists $\delta^{*}>0$ such that $\hat{\omega}_{j}^{\prime \prime}:=\phi_{j}\left(\omega_{j}^{\prime \prime}\right)$ satisfies

$$
\left\{t \zeta: \zeta \in \hat{\omega}_{j}^{\prime \prime}\right\} \subset \hat{\omega}_{j}, \quad \forall j, \forall t \in\left[1-\delta^{*}, 1\right]
$$


Indeed, let $\phi_{j}\left(x_{j}\right)=\left(1, \ldots, 1, \tilde{x}_{j}\right)$ with $\tilde{x}_{j} \in \mathbf{R}^{2 n-m_{j}}$. We can take

$$
\hat{\omega}_{j}^{\prime \prime}=\left(1-\delta^{*}, 1+\delta^{*}\right)^{m_{j}} \times B_{2 n-m_{j}}^{\delta^{\prime \prime}}\left(\tilde{x}_{j}\right)
$$

where $B_{2 n-m_{j}}^{\delta^{\prime \prime}}\left(\tilde{x}_{j}\right)$ is the ball in $\mathbf{R}^{2 n-m_{j}}$ centered at $\tilde{x}_{j}$ with a sufficiently small radius $\delta^{\prime \prime}$. Note that

$$
\phi_{j}\left(Q^{\rho} \cap \omega_{j}^{\prime \prime}\right)=\left(1-\delta^{*}, \rho_{1}\right) \times \cdots \times\left(1-\delta^{*}, \rho_{m_{j}}\right) \times B_{2 n-m_{j}}^{\delta^{\prime \prime}}\left(\tilde{x}_{j}\right) .
$$

Define

$$
M^{*}=\sup \left\{|f(z)|: z \in Q \backslash \cup_{j=1}^{k} \omega_{j}^{\prime \prime}\right\}
$$

Then $M^{*}<1$. By the maximum principle, we have $|f| \leq M^{*}$ on $Q \backslash \cup_{j=1}^{k} \omega_{j}^{\prime \prime}$. Fix $r_{*}$ so that

$$
1>r_{*}>\max \left\{1-\delta^{*}, M^{*}, M_{1}, \ldots, M_{k}\right\} .
$$

Suppose that $r_{*} \leq \rho_{i}^{\prime} \leq \rho_{i} \leq 1$ for $i=1, \ldots, N$. Let $\Omega$ be a connected component of $Q_{N}^{\rho}$. Since $\Omega$ does not have a compact connected component, there exists $z^{*} \in \partial \Omega$ satisfying $\left|f_{i}\left(z^{*}\right)\right|=\rho_{i}$ for some $i$. Since $\rho_{i}>M^{*}$, then $z^{*} \in \omega_{j}^{\prime \prime}$ for some $j$. Let us assume that $z^{*} \in \omega_{1}^{\prime \prime}$, and $\left(\mu_{1,1}, \ldots, \mu_{1, m_{1}}\right)=\left(1, \ldots, m_{1}\right)$. Thus $\phi_{1}=\left(\left|f_{1}\right|, \ldots,\left|f_{m_{1}}\right|, \tilde{f}_{m_{1}+1}, \ldots, \tilde{f}_{2 n}\right)$. We now replace $z^{*}$ by some $z_{*} \in \Omega \cap \omega_{1}^{\prime \prime}$. We consider a path defined by

$$
t \rightarrow \gamma(t):=\phi_{1}^{-1}\left(t \phi_{1}\left(z_{*}\right)\right), \quad 1-\delta^{*} \leq t \leq 1 .
$$

Note that by (A.43), $\gamma$ is well defined and is contained in $\omega_{1}$. We now have

$$
\left|f_{\ell}(\gamma(t))\right|=t\left|f_{\ell}\left(z_{*}\right)\right| \leq t \rho_{\ell}, \quad \ell \leq m_{1} .
$$

Since $\gamma(t) \in \omega_{1}$, we also have

$$
\left|f_{\ell}(\gamma(t))\right| \leq M_{1}<r_{*}, \quad \ell>m_{1} .
$$

This shows that $\gamma(t) \in Q_{N}^{\rho}$. Since $\Omega$ is a connected component of $Q_{N}^{\rho}$ and $\gamma(1)=z_{*} \in \Omega$, we must have $\gamma(t) \in \Omega$. By the definition of $M_{j}$, at $t=1-\delta^{*}$ we have $t \rho_{\ell} \leq 1-\delta^{*}<\rho_{\ell}^{\prime}$. Combining with (A.46)-(A.47), we get $\gamma\left(1-\delta^{*}\right) \in Q_{N}^{\rho^{\prime}}$.

(b) Since $p_{0}, p_{1}$ are in the same $\hat{\omega}_{j}^{\prime \prime}$, the assertion also follows from the above construction of $\hat{\omega}_{j}^{\prime \prime}$ via (A.44)-(A.45) and the convexity of $\hat{\omega}_{j}^{\prime \prime}$.

In summary, by Proposition A.19 we cover $C$ by generic analytic $n$-polyhedrons $U_{i}=$ $\varphi_{i}^{-1}\left(\Delta_{n}\right)(i=1, \ldots, m)$, which are in the general position. By Lemma A.16, each $U_{i} \cap U_{j}$, if non-empty, is a generic analytic polyhedron. Applying Proposition A.20 (a) to all nonempty $U_{i} \cap U_{j}$, we know that $\left\{U_{i}^{r}=\varphi_{i}^{-1}\left(\Delta_{n}^{r}\right): i=1, \ldots, m\right\}$ for $r_{*} \leq r \leq 1$ is a family of nested coverings. Therefore, we can apply Theorem A.9 and Theorem A.12.

\section{REFERENCES}

[1] P. Ahern and X. Gong, A complete classification for pairs of real analytic curves in the complex plane with tangential intersection, J. Dyn. Control Syst. 11 (2005), no. 1, 1-71. MR2122466

[2] V. I. Arnol'd, Bifurcations of invariant manifolds of differential equations, and normal forms of neighborhoods of elliptic curves, Funkcional. Anal. i Priložen. 10 (1976), no. 4, 1-12. MR0431285

[3] _ Geometrical methods in the theory of ordinary differential equations, Second, Grundlehren der Mathematischen Wissenschaften [Fundamental Principles of Mathematical Sciences], vol. 250, Springer-Verlag, New York, 1988. Translated from the Russian by Joseph Szücs [József M. Szücs]. MR947141 
[4] A. D. Brjuno, Analytic form of differential equations. I, II, Trudy Moskov. Mat. Obšč. 25 (1971), 119-262; ibid. 26 (1972), 199-239. MR0377192

[5] C. Camacho, H. Movasati, and P. Sad, Fibered neighborhoods of curves in surfaces, J. Geom. Anal. 13 (2003), no. 1, 55-66. MR1967036

[6] B. Claudon, F. Loray, J. V. Pereira, and F. Touzet, Compact leaves of codimension one holomorphic foliations on projective manifolds, Ann. Sci. École Norm. Sup. (4) 51 (2018), 1457-1506.

[7] M. Commichau and H. Grauert, Das formale Prinzip für kompakte komplexe Untermannigfaltigkeiten mit 1-positivem Normalenbündel, 1981, pp. 101-126. MR627752

[8] I. F. Donin, Cohomology with estimates for coherent analytic sheaves over complex spaces, Mat. Sb. (N.S.) 86(128) (1971), 339-366. MR0299829

[9] E. Fischer, Über die Differentiationsprozesse der Algebra, J. Reine Angew. Math. 148 (1918), 1-78. MR1580952

[10] M. Golubitsky and V. Guillemin, Stable mappings and their singularities, Springer-Verlag, New YorkHeidelberg, 1973. Graduate Texts in Mathematics, Vol. 14. MR0341518

[11] X. Gong and L. Stolovitch, Real submanifolds of maximum complex tangent space at a CR singular point, I, Invent. Math. 206 (2016), no. 2, 293-377. MR3570294

[12] H. Grauert, Über Modifikationen und exzeptionelle analytische Mengen, Math. Ann. 146 (1962), 331368. MR0137127

[13] H. Grauert and R. Remmert, Coherent analytic sheaves, Grundlehren der Mathematischen Wissenschaften [Fundamental Principles of Mathematical Sciences], vol. 265, Springer-Verlag, Berlin, 1984. MR755331

[14] — Theory of Stein spaces, Classics in Mathematics, Springer-Verlag, Berlin, 2004. Translated from the German by Alan Huckleberry, Reprint of the 1979 translation. MR2029201

[15] P. A. Griffiths, The extension problem in complex analysis. II. Embeddings with positive normal bundle, Amer. J. Math. 88 (1966), 366-446. MR0206980

[16] P. Griffiths and J. Harris, Principles of algebraic geometry, Wiley Classics Library, John Wiley \& Sons, Inc., New York, 1994. Reprint of the 1978 original. MR1288523

[17] H. Hironaka and H. Rossi, On the equivalence of imbeddings of exceptional complex spaces, Math. Ann. 156 (1964), 313-333. MR0171784

[18] A. Hirschowitz, On the convergence of formal equivalence between embeddings, Ann. of Math. (2) 113 (1981), no. 3, 501-514. MR621013

[19] L. Hörmander, $L^{2}$ estimates and existence theorems for the $\bar{\partial}$ operator, Acta Math. 113 (1965), 89152. MR0179443

[20] — An introduction to complex analysis in several variables, Third, North-Holland Mathematical Library, vol. 7, North-Holland Publishing Co., Amsterdam, 1990. MR1045639

[21] J.-M. Hwang, An application of Cartan's equivalence method to Hirschowitz's conjecture on the formal principle, Annals of Math. 189 (2019), no. 3, 945-978.

[22] Yu. S. Ilyashenko and A. S. Pjartli, Neighborhoods of zero type imbeddings of complex tori, Trudy Sem. Petrovsk. 5 (1979), 85-95. MR549623

[23] G. Iooss and E. Lombardi, Polynomial normal forms with exponentially small remainder for analytic vector fields, J. Differential Equations 212 (2005), no. 1, 1-61. MR2130546

[24] K. Kodaira, A theorem of completeness of characteristic systems for analytic families of compact submanifolds of complex manifolds, Ann. of Math. (2) 75 (1962), 146-162, DOI 10.2307/1970424. MR0133841

[25] K. Kodaira and D. C. Spencer, A theorem of completeness of characteristic systems of complete continuous systems, Amer. J. Math. 81 (1959), 477-500. MR0112156

[26] T. Koike, Toward a higher codimensional Ueda theory, Math. Z. 281 (2015), no. 3-4, 967-991. MR3421649

[27] S. Kosarew, Ein allgemeines Kriterium für das formale Prinzip, J. Reine Angew. Math. 388 (1988), 18-39. MR944181 
[28] E. Lombardi and L. Stolovitch, Normal forms of analytic perturbations of quasihomogeneous vector fields: rigidity, invariant analytic sets and exponentially small approximation, Ann. Sci. Éc. Norm. Supér. (4) 43 (2010), no. 4, 659-718. MR2722512

[29] F. Loray, O. Thom, and F. Touzet, Two dimensional neighborhoods of elliptic curves: formal classification and foliations, arXiv e-prints (April 2017), available at 1704.05214.

[30] J. Morrow and H. Rossi, Submanifolds of $\mathbf{P}^{n}$ with splitting normal bundle sequence are linear, Math. Ann. 234 (1978), no. 3, 253-261. MR0492406

[31] A. Neeman, Ueda theory: theorems and problems, Mem. Amer. Math. Soc. 81 (1989), no. 415.

[32] L. Nirenberg and D. C. Spencer, On rigidity of holomorphic imbeddings, Contributions to function theory (Internat. Colloq. Function Theory, Bombay, 1960), 1960, pp. 133-137. MR0125982

[33] T. Ohsawa, $L^{2}$ approaches in several complex variables, Springer Monographs in Mathematics, Springer, Tokyo, 2015. Development of Oka-Cartan theory by $L^{2}$ estimates for the $\bar{\partial}$ operator. MR3443603

[34] J. Pöschel, On invariant manifolds of complex analytic mappings near fixed points, Exposition. Math. 4 (1986), no. 2, 97-109. MR879908

[35] H. Rüssmann, Stability of elliptic fixed points of analytic area-preserving mappings under the Bruno condition, Ergodic Theory Dynam. Systems 22 (2002), no. 5, 1551-1573. MR1934150

[36] V. I. Savel'ev, Zero-type imbedding of a sphere into complex surfaces, Vestnik Moskov. Univ. Ser. I Mat. Mekh. 4 (1982), 28-32, 85. MR671883

[37] J.-P. Serre, Prolongement de faisceaux analytiques cohérents, Ann. Inst. Fourier (Grenoble) 16 (1966), no. fasc. 1, 363-374. MR0212214

[38] H. S. Shapiro, An algebraic theorem of E. Fischer, and the holomorphic Goursat problem, Bull. London Math. Soc. 21 (1989), no. 6, 513-537. MR1018198

[39] L. Stolovitch, Singular complete integrability, Inst. Hautes Études Sci. Publ. Math. 91 (2000), 133-210 (2001). MR1828744

[40] T. Ueda, On the neighborhood of a compact complex curve with topologically trivial normal bundle, J. Math. Kyoto Univ. 22 (1982/83), no. 4, 583-607. MR685520

[41] J.-C. Yoccoz, Petits diviseurs en dimension 1, Astérisque 231 (1995).

[42] K. Zhu, Spaces of holomorphic functions in the unit ball, Graduate Texts in Mathematics, vol. 226, Springer-Verlag, New York, 2005. MR2115155

Department of Mathematics, University of Wisconsin-Madison, Madison, Wi 53706, U.S.A.

E-mail address: gong@math.wisc.edu

CNRS and Laboratoire J.-A. Dieudonné U.M.R. 7351, Université Côte D’Azur, Parc Valrose 06108 Nice Cedex 02, France

E-mail address: stolo@unice.fr 